\title{
A tailored wellness intervention for college students using internet-based technology
}

\author{
Alessandro Quartiroli \\ West Virginia University
}

Follow this and additional works at: https://researchrepository.wvu.edu/etd

\section{Recommended Citation}

Quartiroli, Alessandro, "A tailored wellness intervention for college students using internet-based technology" (2011). Graduate Theses, Dissertations, and Problem Reports. 3409.

https://researchrepository.wvu.edu/etd/3409

This Dissertation is protected by copyright and/or related rights. It has been brought to you by the The Research Repository @ WVU with permission from the rights-holder(s). You are free to use this Dissertation in any way that is permitted by the copyright and related rights legislation that applies to your use. For other uses you must obtain permission from the rights-holder(s) directly, unless additional rights are indicated by a Creative Commons license in the record and/ or on the work itself. This Dissertation has been accepted for inclusion in WVU Graduate Theses, Dissertations, and Problem Reports collection by an authorized administrator of The Research Repository @ WVU.

For more information, please contact researchrepository@mail.wvu.edu. 
A tailored wellness intervention for college students using internet-based technology

\author{
Alessandro Quartiroli, M.S. \\ Dissertation submitted to \\ The College of Physical Activity and Sport Sciences \\ at West Virginia University \\ in partial fulfillment of the requirements for the degree of \\ Doctor of Philosophy \\ in \\ Kinesiology \\ with a major in Sport and Exercise Psychology
}

Samuel Zizzi, Ed.D., Chair Christiaan Abildso, Ph.D. MPH

Bill Reger-Nash, Ed.D.

T. Anne Hawkins, Ph.D.

Vanessa Shannon, Ph.D.

Morgantown, WV

2011

Keywords: Freshmen College Students, Physical Activity, Nutrition, Alcohol Use, Internet, Expert Systems 


\begin{abstract}
A tailored wellness intervention for first year college students using internet-based technology Alessandro Quartiroli
\end{abstract}

Objective: The purpose of this study was to develop and implement a theory-based, computertailored feedback system for freshman students at a large, public university. The objective was to understand its potential effectiveness in helping the university in enhancing student wellness.

Participants: Sixty-two college students, out of the over 1,300 contacted, completed the eight week intervention.

Methods: The participants were randomly assigned into two groups and received the survey three times, consistently receiving normative or personalized feedback.

Results: Mainly constituted by freshman, Caucasian, and normal weight individuals, the participating sample was generally healthy. Repeated-measure ANOVAs were run and some small significant differences were found in the changes happened in the interactions between the received feedback and the amount of moderate physical activity, the number of drinks per day and the likelihood to engage and the importance attributed to moderate drinking behaviors.

Conclusions: This study showed potential preventive aspects of this intervention, which could help institutions in supplying preventive helpful services, and eventually delivering "curative" health services related to unhealthy diet, drinking habits, or inactive lifestyle. However, ceiling effects present in all dependent variables and the short time frame of the intervention limited its effectiveness in changing attitude or behaviors.

Keywords: College Students, physical activity, nutritional, and drinking habits, Internet, tailored feedback 


\section{DEDICATION}

Mom and Dad

Because, no matter how far we are from each other, how tired we are from our struggles, how difficult is to be a team sometimes, you never stop to be the most wonderful example I could ever be inspired by.

$\underline{\text { Andrea }}$

With your personal style, you always show me how great and wonderful is to know that I will always be able to count and rely on you. I know you will be wonderful in showing that also to Alessandra and Daniele, new and wonderful pieces of your and our lives.

VI VOGLIO BENE!!!! 


\section{Acknowledgements}

A big thank you to my committee members! I really appreciated your support in developing this project and in teaching me how to face, with the right attitude, all the barriers during the journey.

Dr. Zizzi, Liz, Addy, and Samantha I will not say thank you enough for having shared so much together during these years and for having made me part of your life. Dr. Zizzi, thank you for having believed in me. Thank you for spreading the seeds, now it is Ale's time to make the wine! I tried hard to meet your expectations; I will get there, little by little. I would not have got here without you. July 2004 will always have a special meaning for me. Thank you for the journey

Christiaan, Laurie, Max, and Tessa, thank you so much for everything you have done to enrich my journey and to have shown me how to make Morgantown home. Christiaan thank you for having accompanied me during this journey, not only in completing it.

I want to thank Dr.Reger-Nash and Jan. In one week you have changed in many ways my attitude toward many things, in addition to have shared your wise perspective. And you still do...

Dr. Hawkins, your mail meant the world to me. I might still have to learn it, but I feel I am on the right path, now is just a matter of keep walking. You know what I mean.....

Dr. Shannon, thank you for having shared your teaching wisdom with me!

Thank you to Jessica who has been wonderful support and great friend during this journey. It has been fun to walk this path together!!!! Justine, thank you for your consistent support and peer mentorship also from far away. You ladies have made the difference!

A big hug to Marta, constantly and consistently my fan! I am yours! Your support and invaluable friendship mean the world to me

A huge "thank you" to John! You showed me a wonderful way to be Ale and to be a good family and community man. I hope to get there one day. Thank you to Jane, Katie, and Julian, because with John, you opened your house to me and have made me part of your family.

Fabio and Danielle. It is hard to think all these years without you guys. I really have no many words. Between ups and downs, we built together something that nothing and nobody will be ever able to take away from us. Fabio....GRAZIE!

Dr.Watson, I will never be able to say thank you enough for having made me understand the beauty of be enthusiastically involved. It meant a lot to me. You taught me more than just being an academic.

Dr. Etzel, thank you for having taught me, directly and indirectly, the wonderful and truthful meaning of compassion and welcoming. I will always keep them in my mind.

Damien, thank you for listening when needed and to have worked with me on my applied skills 
Don Ambrogio, grazie per il constante e sempre presente supporto!

Thank you to Marialuisa, Caterina, Daniela, and all UCSC people who have supported me along the way.

Aurelio y David, mis hermanos, aun si lejos siempre estáis en mi corazón. Gente especial con la que he compartido momentos estupendos y que nunca olvidaré.

Michelangelo, Niki, Marco, Alessandro fellow travelers in this journey. It was awesome to share joys and difficulties together. Our brotherhood did not stop in Morgantown...

Dr.Tunietti, Ud y familia han estado fantásticos amigos y compañeros de viaje. Muchísimas gracias por la confianza y el apoyo que siempre os me habéis ofrecido

Deb and Pete thank you for having always been ready to open the doors of your house. Deb, thank you for having being joyful and consistent support.

Olivier, "Chef du Village", keep up with the European style! Thank you for being you!

Claudio, you showed me what friendship really means, small gestures, but constantly expressed. Thank you for having been part of my life-journey.

Carla your caring has always been more than important! Thank you!

MaryAudrey and Micheal, in these two years you guys taught me and share with me something that will always stay with me ...Thank you for all you have done and you have been for me!

Dr.Kasprowicz and Dr.McClung if I have found what I needed to get here a big part is because of you. Your compassion and support have been essential in the last rush of this ride. Thank you!

Un grazie di cuore a Rina e Severino sempre vicini e di supporto durante il mio cammino!

Ambrogio Rovelli, Ambrogio Restelli, Giulio Parravici, it is great to know that no matter how often I will be home, but I will always find warm and gentle support in you and in your families.

A gigantic THANK YOU to all my players throughout the years....you taught me way more than I taught to you guys. Sometimes we realize it only with time. I will never forget it. With your smile and your enthusiasm you shaped my joy for being Ale! Thank you!

Thank you to Meagan, Marissa, Kevin, and all the SEP students who helped me with the project!

Many more are the people that deeply influenced my journey! I believe that is all about teamwork and I have been so lucky to have a wonderful team supporting me in this journey, helping me to be who I am and to walk through my path. Thank you to all of you, those who I mentioned and those I did not. You all have kept supporting me regardless the difficulties. You all often reminded me that I could make it and reminded me who I am, also when I was forgetting it. 


\section{TABLE OF CONTENTS}

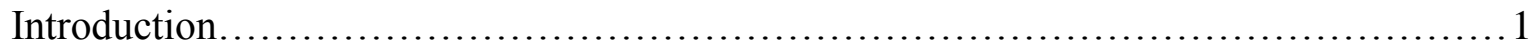

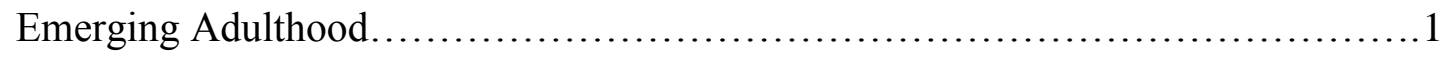

Young Adult Health Patterns.............................................. 2

Internet Use of the College Student Population................................ 3

Knowledge-Based Systems (KBS) and Health Behavioral Change Interventions......4

Purpose................................................................5

Research Questions....................................................6

Methods.......................................................................... 7

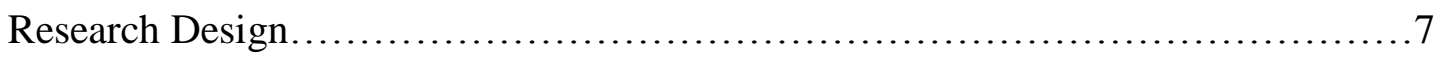

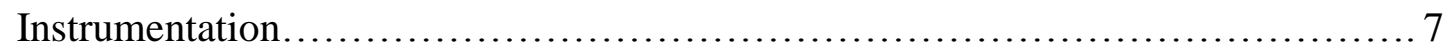

Data Collection Procedures............................................. 8

Participant Recruitment and Data Collection............................... 8

Initial recruitment................................................ 9

The follow up protocol...........................................10

The final sample - The Intervention sub-sample....................... 10

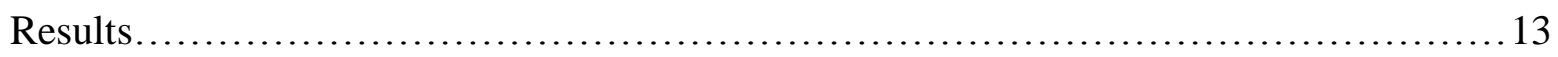

Characteristics of the General Sample...................................... 13

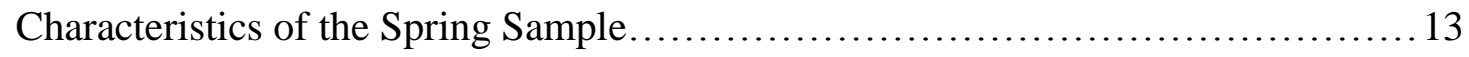

Characteristics of the Intervention Sub-sample............................. 14

Attitude and Behavior Change During the Intervention......................... 15

Qualitative Evaluation of the Intervention................................. 17

Intervention Costs......................................................... 19 
Discussion.............................................................. 20

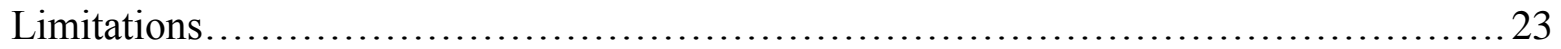

Conclusion and Future Directions........................................... 24

References ................................................................. 28

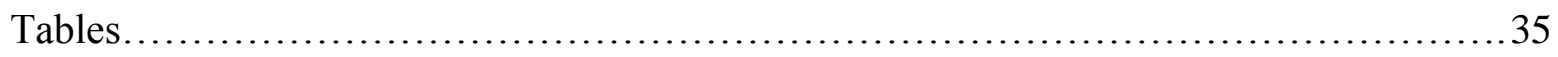

Figures...............................................................41

Appendixes.............................................................. 42

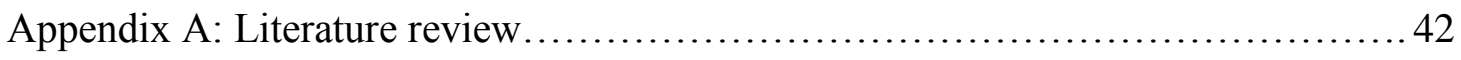

Appendix B: Distinctions between the three administrations...................... 165

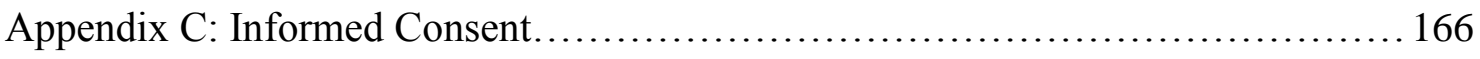

Appendix D: The three versions of the survey.............................. 168

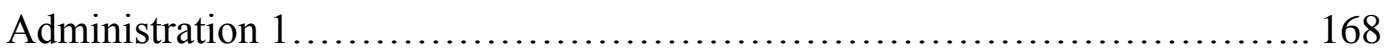

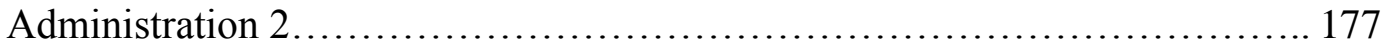

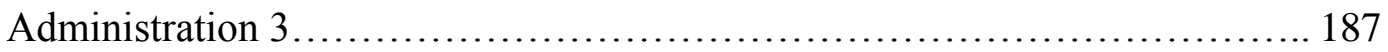

Appendix E: Structure of the Personalized Feedback: Keys and Content............ 197

General Health Feedback............................................ 197

Physical Activity................................................. 198

Nutrition......................................................... 204

Alcohol................................................................209 


\section{List of Tables}

Table 1: Demographics of the three samples

Table 2. Percentage of Individuals meeting guidelines of health behaviors in the

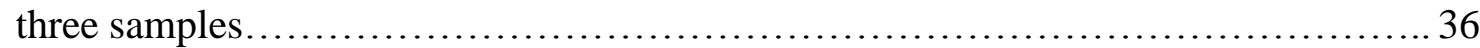

Table 3. Descriptive data related to the TPB, the TTM, and the health behavioral

habits of the WVU samples, divided by Samples................................ 37

Table 4. Comparison of health behaviors and TBP variables reports between

personalized and normative feedback, along the three administrations.................38

Table 5. Comparison of the data related to the health behaviors, the TBP and the

TTM of the complete sample participating in the study, looking at the time points.....39

Table 6. Comparison of the data related to the health behaviors and the TTM of the complete sample participating in the study, looking at the time points.... 


\section{List of Figures}

Figure1: Sampling/Recruitment Flow Chart....................................41 


\section{Introduction}

College students, once considered a healthy and low risk population, have shown vulnerability to weight gain due to the development of unhealthy behaviors, such as physical inactivity, poor diet, and alcohol misuse. The 1995 National College Health Risk Behavior Survey (NCHRBS) found that $20.5 \%$ of college students were overweight (Lowry, Galuska, Fulton, Wechsler, Kann, \& Collins, 2000) and more recent data suggest this number has climbed substantially (Ferrara, Laurent, and Wilson, 2008). In fact, the greatest increase in overweight and obesity in adults has been found between 18 and 29 years of age (BRFSS: CDC, 2007; Racette, Deusinger, Highstein, Strube, \& Deusinger, 2005). Despite evidence showing short- and long-term health repercussions in this emerging adulthood population (Lien, Lytle, Klepp, 2001; Kvaavik, Andersen, \& Klepp, 2005), this sub-group has received far less attention in the literature than adolescents (Park, Mulye, Adams, Brindis, \& Irwin, 2006).

\section{Emerging Adulthood}

Emerging Adulthood (Arnett, 2000) is a transitional stage between adolescence and full adulthood, considered to be a period of profound change and importance in the life of an individual (Medalie, 1981). During this life stage, individuals are characterized by potential engagement in risky behaviors, such as alcohol misuse and smoking (Kelder, Perry, Klepp, \& Lytle, 1994; Ravert, 2009). The development of self-identity takes priority as individuals explore new ideologies and behaviors. Within this process of identity development, researchers suggest that healthy lifestyle values and behaviors can be formalized and can be precursors of adult behaviors (Miller, Ogletree, \& Welshimer, 2002; Storer, Cychosz, \& Anderson, 1997).

Obesity and weight maintenance have become important public health issues, and subsequently, methods to intervene during key life periods of weight gain have gained attention 
(Anderson, Shapiro, \& Lundgren 2003). Since a large amount of emerging adults (18.4 million students) are enrolled in college and the potential persistency of some of the "college behaviors" to continue into adulthood (Kelder et al., 1994; McCracken, Jiles, \& Blanck 2007), this time period may be ripe for intervention, offering a potentially wide public health impact.

\section{Young Adult Health Patterns}

Keating, Guan, Castro Piñero, and Bridges (2005) stated that among the college population, $50 \%$ was engaged in physical activity, with no more than $36 \%$ of these students meeting the adequate amount of PA suggested by ACSM and CDC. Based on these findings, they suggested higher education as one of the best contexts for addressing the PA behaviors, with the possibility to develop interventions specifically focused on this "captive" audience.

In terms of meeting the recommended dietary guidelines, Anding, Suminski, and Boss (2001) reported that $43 \%$ of the college women in their study followed at least one of the considered dietary guidelines, whereas $32 \%$ and $10 \%$ of this population followed respectively two or three of them. Other studies observed only one third of college students consuming the recommended serving of fruit and vegetables (Racette et al., 2005).

Schröder et al (2007) found that alcohol, and specifically the consumption of more than 3 drinks of alcohol, (along with other factors such as, smoking, educational level, leisure- time physical activity, energy, and diet quality) was significantly associated with the risk of abdominal obesity. In terms of the presence of alcohol among this population, the CORE Institute (2005) showed that drinkers constitute $85 \%$ of the US college population with $26 \%$ considered heavy (five or more drinks -four for women- at least five times in a month) and frequent (an average of three or more occasions per week) drinkers. Because of the high caloric 
intake from alcoholic beverages and fast food, college students often are engaged in nutritional behaviors to control their weight (Nelson, Story, Larson, Neumark-Sztainer, \& Lytle, 2008).

Hull, Morrow, Dinger, Han, and Fields (2007) found that in college students the average gain weight was approximately 4 lbs during their first freshman year, nearly 5 times the reported weight gained averagely by for the general population (Mihalopoulos, Auinger, \& Klein, 2008). To address this phenomenon and the health behaviors in this specific population, it has been thought that the use of alternative tools and instruments technology could help engage these emerging adults.

\section{Internet Use of the College Student Population}

The U.S. Department of Commerce (2004) report shows that $70.7 \%$ of the general population between 18 and 24 years old, among whom $86.7 \%$ were students, uses the internet. In recent years the Internet has begun to be considered a useful tool to gather health related information (i.e. Hargittai \& Hinnant, 2008; Powell, Darvell, \& Gray, 2003), and consequentially also as a mean to develop, implement, and deliver behavioral change interventions. Some of the advantages of the Internet as a medium for intervention include the opportunity to reach a wide range of people in different locations at a low cost, and the possibility to provide personalized and interactive feedback, without the necessity of face-to-face contact (Kreuter, Strecher, \& Glassman, 1999). The effectiveness of the Internet in delivering behavioral change interventions has been tested by many authors (Brunsting \& van den Putte, 2006; Freemantle, Gill, Godfrey, Long, Richards, Sheldon, et al., 1993), showing its promising benefits (i.e., Kroeze, Werkman, \& Brug, 2006; Vandelanotte, Spathonis, Eakin, \& Owen, 2007).

McTigue, Garrett, and Popkin (2002) addressed the necessity of an early intervention for health behaviors, because of the fact that health-risks, generally established during adolescence 
and young adulthood, are also generally maintained throughout adulthood (McCracken, et al. 2007).

\section{Knowledge-Based Systems (KBS) and Health Behavioral Change Interventions}

To develop and provide the aforementioned interventions, some researchers have started to use knowledge-based systems (KBS). KBS are software that achieve expert-level competence in solving problems in a specific task domain. These systems, using self-reported questionnaire data, develop individualized feedback that addresses the individuals' needs and the current behaviors (Engelmore \& Feigenbaum, 1993). If used within the realm of health behavioral change and delivered via Internet, KBS would overcome common barriers such as time and accessibility to professionals and help successfully perform these interventions, (Lustria, Cortese, Noar, \& Glueckauf, 2009).

The effectiveness of KBS in behavioral change interventions has been proven in the realm of physical activity (Marcus, Williams, Dubbert, Sallis, King, \& Yancey, et al., 2006; Spittaels, De Bourdeaudhuij, Brug, \& Vandelanotte, 2007), nutrition (Vandelanotte et al., 2005), alcohol (Weitzel, Bernhardt, Usdan, Mays, \& Glanz, 2007), and nicotine (Strecher, Shiffman, \& West, 2005).Newton, Kim, and Newton (2006) developed a study designed for freshmen students enrolled in orientation freshman class at a large Midwestern university, within a program called PAC-CATS (Personal Assessment Challenge to Choose Activities for Today's Success). This study used a health behavior survey to assess, and provide feedback on, three health behaviors (PA, eating habits, and stress management). The sample included 695 students (306 males and 387 females). After the administration of the survey, selected classes (450 students) received a 50 minutes oral presentation about the need of a healthy lifestyle and about 
the program itself. The participating students were recruited and divided into two groups, receiving a different option of the intervention..

The first group received an 8-week intensive behavioral change program (“IBCP”), accompanied by a peer-mentoring program and academic incentives ( 85 students). The second group, instead, had access to an educational intervention ("EI") with individualized selfmonitoring and some incentives (148 students). One hundred and thirty eight students completed the entire intervention (63 in "IBCP group" and 75 in "EI group"). The authors found some differences between the groups, such as different levels of readiness of change, which increased in $44.4 \%$ of the students in "IBCP group" compared to $37.3 \%$ of the students in option 2 . The "IBCP group" showed significant behavior gains in 13 out of the total 17 addressed health behaviors, whereas the "EI group" only had gains in four. This study provides some evidence that computer generated feedback offered over a short time frame can potentially impact student health attitudes and behaviors.

College students are often considered an “online population” (U.S. Department of Commerce, 2004), because of the high frequency of college students (72\%) online (Anderson, 2001), and because of the fundamental role played by the Internet in their lives (Ascribe Newswire, 2002). For these reasons, reaching those college students through the Internet world could potential increase the use and effectiveness of health interventions.

\section{Purpose}

In terms of outcomes, this study was designed to develop and implement a theory-based, computer-tailored feedback system for students at a large, public university in the United States. Specifically, the desired outcomes of the intervention included increases in the following self- 
reported behaviors; 1) fruit and vegetable intake; 2) moderate and vigorous physical activity, and the decreases in 3) binge and heavy drinking episodes.

\section{Research Questions}

Descriptive:

- How are the considered behaviors of the students participating in the study characterized at the beginning and at the middle of their second semester characterized?

- What level of intention to behave and readiness to change characterizes the participating students at the beginning and at the middle of the intervention?

Impact of the Intervention

- Did the use of an internet-based knowledge based system, providing student individual tailored feedback, affect the health behavioral habits of this population?

- How did the measured health behaviors change over time, and what impact, if any, did the different levels of the intervention play in these changes?

- How did the level of intention to behave and readiness of change, change over time, and what impact, if any, did the different levels of the intervention play in these changes?

Evaluation of the intervention

- Do participants perceive the system useful and useable? 


\section{Methods}

\section{Research Design}

The design of this study is a pseudo experimental design without a true control group. All participants recruited were randomly assigned to one of two intervention conditions: 1) Personalized feedback group (PERS); 2) Normative informational feedback (NORM).

\section{Instrumentation}

The theoretical frameworks of the questionnaire were the Transtheoretical Model of Change (TTM; Prochaska, \& DiClemente, 1982; 1983) and the Theory of Planned Behavior (TPB; Ajzen, 1985; 1991). Using these theories for the structure and Dillman's (2007) principles of tailored design, specific items were then built based on previous health behavior surveys (Newton et al., 2006; Bartlett, 2009; CDC's Behavioral Risk Factor Surveillance System, 2009).

The survey's pool of questions had a total 80 items across four main sections: Introduction (3 questions), Physical Activity Behaviors (12 questions), Eating Behaviors (14 questions), and Drinking Behaviors (12 questions). The three behavior-specific sections were similar in their structure, while the other sections were alternatively presented during the three administrations (APPENDIX B). Five main sections characterized each portion of the survey. Each one of the behavioral sections include behavioral descriptive questions (between 4 and 9 items); a stage of readiness item (1 item), a question focusing on intentions to behave (1 item), items about different barriers ( 6 - 7 items), items about the individual perceptions of the general student body behavior ( $1-4$ items), and eventually, if necessary, follow up TPB related questions (3 items). The first described the level of engagement in the specific behavior (e.g., "In a usual week how many days do you do 30 or more minutes of moderate activity (only count bouts of at least 10 minutes)?"). These questions were characterized by answer options targeting 
behaviors on a ratio scale. The second addressed the individual's readiness of change regarding this behavior (e.g., "Which of these statements most closely reflects you in your exercise behavior?"), characterized by a multiple choice answer whose each level characterized one of the five stages of change. The third section analyzed the individual's intention to behave (e.g., "I will eat healthy in the next month" and "How important is it for you to maintain a moderate drinking"), and the individuals could answer using a 6-points Likert scale answering option. The fourth subsections looked at the individual's perception of the general WVU student population characteristics within the specific behavior (e.g., "In the last month, in your opinion, how many days does an average WVU student drink?") with possible answers focusing on the weekly or monthly frequency of the targeted behaviors on a ratio scale. The last section looked at the barriers preventing the engagement in these behaviors (e.g., "Which of the following barriers interfere with or prevent you from eating healthy?"), and the answer were a yes-no dichotomous answering options.

The different administrations of the questionnaire had some core questions constantly repeated, while other questions, addressing specific topics, were administration-specific (APPENDIXES B \& D). The core of the administrations moved between 54 and 58 questions, while the additional 9 questions were asked following a score equal or less than 4 to the question about the likelihood to engage in the behavior (i.e. "I will exercise regularly in the next month").

A draft of the survey, including the entire pool of items, was piloted during the spring 2010 in a general introductory class with 8 first year students. This group of students completed it in an average time of 14 minutes, providing generally positive feedback. 


\section{Data Collection Procedures}

Prior to collecting data, approval was obtained from the West Virginia University Institutional Review Board (IRB) for the Protection of Human Subjects. The informed consent (Appendix C) was provided in a cover letter to the participants when they clicked on the provided link. In the cover letter, the participants were provided with information about the study, about the approval by the IRB and about the possibility to not answer all questions and/or to decline the participation to the study.

All participants were contacted by email and asked to complete the three administrations of the questionnaire. The students in the PERS group received personalized feedback, developed by the KBS, after the completion of all three administrations. Instead, the participants in the NORM group were provided with normative feedback, which was developed by the researcher and based on the Guidelines for Americans and scientific literature. The NORM feedback was equal for all the participants in this group. The researcher, who analyzed all the possible combinations of answers that the system could receive, produced for each of them a theory-based paragraph of text, which together formed the personalized feedback (APPENDIX E). The personalized feedback provided the individual with a description of the current behaviors of the individual and also included suggestions to develop, improve, and/or maintain a healthy lifestyle. This personalized feedback was based on nutritional and the physical activity Guidelines for Americans (USDHHS, 2005; USDHHS, 2008), the scientific literature, and the specific information that the participant provided when answering the survey.

\section{Participant Recruitment and Data Collection}

The first administration of the questionnaire was held during the third and fourth weeks of the spring semester $2011(01 / 24-02 / 06)$, the second administration was held during the 
seventh week of the same semester (02/20-02/27), and the last administration was held during the tenth week (03/13-03/20) (Figure 1).

\section{Initial recruitment}

Students were recruited for the study in two different ways. The first recruitment strategy was a "face-to-face" process, and occurred in various residence halls at a large, public university. Trained research assistants asked students for their university-specific email address. Each student was told about the content of the study, the IRB approval, and their eligibility to win a gift certificate. Each evening of the recruitment, the researcher sent out group emails with a brief description of the study and the link for the survey. To avoid spam-walls, these addresses were reached with group-emails, addressing 5 students at a time.

The second recruitment process took place within four major-specific introductory classes, mostly comprised of first-year students. The researcher presented the study in the classes and sent an email to the instructors, who then forwarded the mail to the students through the class e-mail system. The "in-class" approach produced a sample of 566 students. The "face-toface" recruitment produced an initial sample of 955 usable student emails. The total number of students reached with this first round of emails was 1521. After the first round of recruitment, the total potential participant pool was reduced to 1301 potential participants for the entire study after all non-functional emails were removed. The final Spring sample consisted in 41 out of 566 (7.24\%) individuals reached through the "in-class" recruitment, who completed the survey at least once. The "face-to-face" method counted 170 out of 955 (17.8\%) individuals completing the survey at least once. 
In both recruitment protocols the script of the email contacting the students was the same, and when the email was more personalized, according to Dillman (2007), the personalized emails followed the same script, as well.

\section{The follow up protocol.}

The group emails in each of the follow-ups during the study were characterized by as many as nine addresses at a time; the chosen number to avoid "spam-walls." Although the content of the reminder emails was the same in every case, the intensity of their submission was different between the "in class" and the "face-to-face" recruited samples. Students received three reminders for each administration from their instructors' email addresses, while the "face-toface" sample received between 5 and 8 reminders. During the second and the third administrations, the first three reminders were the same for everyone, while the additional reminders, respectively 8 and 5 times, differed between those individuals who already answered to the survey and those who did not. This personalization consisted in addressing the students by name and thanking them for their participation in the previous administration.

\section{The final sample - The Intervention sub-sample .}

After the second administration, the size of the sample of usable data, determined by the willingness of the participants to answer the survey and by a few technical problems created by the online system, was 173 total participants. Among these individuals, 153 were usable for the intervention, whereas twenty participants who took part in the survey could not be included due to a technical error that failed to store their feedback. At the end of the second administration, a total 109 individuals answered the survey, and 81 had previously answered the first administration. 
For the final administration, the class-recruited sample was contacted twice during the administration week, whereas the face-to-face recruited sample was contacted five times, with the frequency of one email every two days. At the conclusion of the third administration, a total sample of 72 individuals answered the survey with 62 who completed all three administrations.

To compare the different sub-samples used during this study, the author will refer to the General sample, when referring to the sample constituted by individuals who completed the survey at least once during the Fall or Spring semester (N=303). The Spring Sample refers to the group of individuals who completed the survey at least once, but only during the spring semester ( $N=211)$, while the intervention sub-sample, will be constituted by those people who completed the intervention, answering the survey at all the three administrations $(\mathrm{N}=62)$. 


\section{Results}

\section{Characteristics of the General Sample}

The general sample $(\mathrm{N}=303)$ included participants who were predominantly White/Caucasian (88.2\%), female (53.8\%), and within a normal weight range (BMI 18.5-24.9) $(57.6 \%)$. Those who completed the survey were most likely freshmen (40.2\%), living in the residence halls $(76.8 \%)$, and reporting living a healthy $(67 \%)$ or very healthy lifestyle $(19.1 \%)$ (Table 1). Healthy individuals, who met the guidelines for all the assessed health behaviors, mostly characterized the general sample. They met guidelines for: moderate physical activity $(50.5 \%)$, vigorous physical activity $(61.1 \%)$, consumption of fruit and vegetables $(86.3 \%)$, moderate (61.1\%), and heavy drinking (75.2\%). Participants also reported positive attitudes and intentions as well as readiness to change. Nearly all attitudinal variables were above the midpoint for the overall sample, showing a "healthy" self-reported profile (Table 2).

\section{Characteristics of the Spring Sample}

The Spring Sample was similar to the general sample in nearly all demographic characteristics. Although it appeared to be very healthy, many cited preventive factors that impeded their engagement in health behaviors. The most frequently reported barriers for these behaviors were: for physical activity, "Lack of time" (73\%), "Having more important things to do" (76.8\%), and "Tiredness" (63.5\%); for healthy diet, "Time constriction" (54.5\%), "Lack of healthy choices" (53.1\%), "Lack of healthy tasty choices on campus" (53.1\%), and "Lack of money" (53.1\%). The highest perceived obstacles to drinking moderately were "Alternative to have fun" (19.4\%) and "Alternative to socialize" (20.9\%). This "healthy" sample showed some poor eating habits, with the majority of participants eating two or more meals per week after 10pm (78.2\%) and $65.4 \%$ of the group skipping breakfast at least twice a week. This group of 
individuals showed fairly low soda consumption with $35.5 \%$ of the individuals not drinking it and $74.9 \%$ with less than a 50cl. bottle a week. Positive results were showed by the low use of fast food restaurants, with $18 \%$ of the sample eating in these restaurants 3 or more times a week. Interestingly, in addition to the health behavior the individuals in this sample engaged in, in terms of alcohol consumption, among them more than half $(53.1 \%)$ did not drink at all.

\section{Characteristics of the Intervention Sub-Sample}

The intervention sub-sample $(n=62)$ was similar to the general sample in nearly all demographic characteristics, although the intervention sample had a slightly higher percentage of women $(60 \%$ to $54 \%)$ and White $(95 \%$ to $88 \%)$ participants. (Table 1$)$.

The intervention sub-sample (Table 2) was characterized by a majority of individuals reporting being already engaged in an active life (45.2\% Maintenance, 19.4\% Action). This data can also be confirmed by this group's self reported data about their ability to meet the guidelines for physical activity (40.3\% meeting moderate, and $69.4 \%$ meeting vigorous guidelines). This group also reported strong importance of a physically active lifestyle $(\mathrm{M}=5.24 ; \mathrm{SD}=0.843)$. In terms of their nutritional habits, one third of the group (19.4\% Maintenance, $11.3 \%$ Action) reported advanced stages of readiness with an additional 51.6\% in Preparation. This data contradicts the self-reported data related to meeting guidelines. A large majority of this group reported meeting the dietary guidelines $(80.6 \%)$ eating five servings of fruit or vegetables a day, and also reported high importance of this behavior $(\mathrm{M}=5.08 ; \mathrm{SD}=0.997)$. Half of the sample $(50 \%)$ considered itself already living a healthy life in terms of their alcohol consumption $(4.8 \%$ Action, 45.2\% Maintenance). This data seems to be confirmed by the self-reported data related to their meeting the drinking guidelines (74.2\% moderate drinking, 95.2\% not heavy drinking). This group rated moderately high both the importance of their engagement in healthy drinking 
behaviors $(M=4.13 ; \mathrm{SD}=1.914)$ and the likelihood of engaging in these behaviors $(\mathrm{M}=4.13$;

$\mathrm{SD}=1.914)$.

\section{Attitude and Behavior Change During the Intervention}

The impact of the intervention was analyzed by running a series of 2 (feedback type) x 3 (time) repeated-measure ANOVAs on each of the dependent variables. In these analyses the Independent variables were the assigned group (Normative Vs. Personalized) and the time points during the intervention (T1, T2, T3), whereas the Dependent variables were: days with moderate physical activity, days with vigorous physical activity, days with stretching, days with strength activity, serving of fruit and vegetable per day, days with at least a drink, numbers of drinks per day, days with 5 or more drinks in a week, number of episodes with 5 or more drinks in a month, likelihood to engage in each of these behaviors and perceive importance of each of these behaviors. Multivariate ANOVAs were not run due to lack of correlations among dependent variables and lower group sizes. However, since multiple ANOVAs were necessary for each set of DVs, the alpha level was set at .01 to control for type I error. See Tables 5 and 6 for descriptive data for the intervention sub-sample, and the changes in self-reported behaviors and attitudes over the eight week period.

In each of the following four reported interactions, the Mauchly's test for sphericity indicated that this assumption was met (Moderate PA: $\mathrm{W}=0.926, \mathrm{df}=2, \mathrm{p}=.103$; Drinks per Day: $\mathrm{W}=0.956, \mathrm{df}=2, \mathrm{p}=.287$; Alcohol Likelihood $\mathrm{W}=0.911, \mathrm{df}=2, \mathrm{p}=.092$; Alcohol Importance $\mathrm{W}=0.982, \mathrm{df}=2, \mathrm{p}=.631$ ), so no corrections were applied to the F-ratio computations. Some small significant effects were found in the interactions between the received feedback variable and a few of the main dependent variables over time. These interaction effects will be discussed in detail below. In the remainder of the two-way models, statistically 
significant main effects or interactions were not found over time for fruit and vegetable intake, vigorous physical activity, or any of the other attitudinal variables across the three health behaviors.

The first significant effect was in the interaction between the type of feedback (Personalized and Normative) and the moderate physical activity variable over time. A small, significant interaction was found $[\mathrm{F}(2,120)=3.93, p=0.02, \mathrm{ES}=0.06$, Obs.Pow $=0.698]$. The normative group showed a slightly bigger change in terms of average days of moderate activity (Personalized 0.23; Normative 0.39). Overall there was not a significant main effect for time $(p=0.314)$, but there was a main effect for the group, which means that there were differences among groups regardless of time effect $[\mathrm{F}(1,60)=4.238, p=0.044, \mathrm{ES}=0.066$, Obs.Pow=0.526]. On average, the Normative group was higher, than the Personalized group on MPA.

In terms of the drinks per day variable, the interaction with the type of feedback, suggests a small, but significant, difference $[\mathrm{F}(2,120)=3.53, p=0.03, \mathrm{ES}=0.058$, Obs.Pow=0.647]. The normative group showed a slightly bigger change, characterized by different directions, in terms of average amount of drinks per day (Personalized -0.14; Normative 0.50). This effect suggests that over time the number of drinks per day decreased in the personalized feedback group, whereas it increased in the normative feedback. Overall, there was not a significant main effect for time $(p=0.238)$ or for group $(p=0.527)$.

Another small interaction effect was found between the level of self-reported likelihood of engaging in healthier drinking and the type of feedback $[\mathrm{F}(2,120)=3.229, p=0.04, \mathrm{ES}=0.058$, Obs.Pow=0.604]. The Personalized group showed a greater change in terms of its likelihood to engage in moderate drinking (Personalized 0.75; Normative -0.43). This effect showed how the individuals in the group receiving the personalized feedback increased their likelihood to drink 
moderately, while the normative feedback group decreased. Overall, there was no significant main effect for time $(p=0.823)$ or for group $(p=0.318)$.

Although not completely significant $(p=0.056)$, the interaction between the importance attributed to moderate drinking and the type of received feedback produced another small effect $[\mathrm{F}(2,120)=2.960, p=0.056, \mathrm{ES}=0.055$, Obs.Pow $=0.565]$. While the personalized feedback maintained the same level of attributed importance, the normative feedback group decreased in this variable $(-0.48)$. Overall, there was not significant main effect for time $(p=0.093)$ or for group ( $p=0.504)$. In all these analyses, due to smaller sample size than expected, the levels of observed power did not reach the needed 0.8 as suggested by Cohen (1988); therefore, the probability of finding a statistically significant difference was lower than desired.

\section{Qualitative Evaluation of the Intervention}

Among the sixty-two individuals who completed the three administrations, 38 commented on what they liked about the intervention, 37 commented on what they did not like, and 36 commented on what they would change. Among the statements related of what they liked, 22 individuals (35.48\%) made positive comments about the feedback and the information provided with it. A couple of examples of these comments were the following statement: "I like the intervention because it opened my eyes to what really goes on" and "forced me to look at my personal lifestyle choices." Those individuals who received the personalized feedback, mostly read it $(77.4 \%)$ and considered it effective (71\%), while those receiving the normative feedback were less likely to have read it (41.9\%), and considered it less useful, with only $38.7 \%$ stating the effective value of the feedback.

Few individuals mentioned that their main motivation to participate in the program and complete the entire study in its three administrations was the promised reward. Other comments 
reflected positively on the fact that the eight weeks intervention "was done over a long period of time." Positive reactions were also found regarding the attention that the intervention raised in comparing the individual's behaviors with those engaged by the general WVU population. One such individual responded by stating "I like that it asked personal questions as well as general questions about WVU students." The easiness of access and the precision of the intervention were also positively considered by individuals in the sample, with participants commenting that it was "simple, to the point, useful" and "very easy to access."

Among the comments about what they disliked about the intervention, seven individuals affirmed to not have disliked anything about it, while ten participants (16\% of the Intervention sub-sample) indicated the length of the survey was too long. Two individuals felt the terms should have been better defined, while one person complained that the survey seemed to make assumptions about alcohol consumption, stating, "I felt like it just assumed that you drink. I don't drink." Two technical comments were made by two individuals who stated that, "It didn't show personalized results based on accumulating data" and that they had trouble submitting the answers. This participant wrote, “There was one time when I filled it out, hit submit, and it didn't go through so I had to redo it. It may have been my computer though."

While nine individuals stated that they would not change anything about the intervention, four suggested a larger variety of questions between administrations. Two individuals suggested that they would shorten the survey and one proposed that two administrations could be sufficient. One subject affirmed the desire of having more health behaviors addressed, whereas another asked for more defined terminology. Interestingly, one individual suggested the potential benefit of having face-to-face interaction in addition to the web-based intervention. 


\section{Intervention Costs}

In terms of the cost of the intervention, the entire delivery cost of the program has been estimated to be around $\$ 2,500$, including the cost of the software and the number of hours used to develop the content of the intervention. The delivery cost per individual, including all of the participants reached by the survey, was around $\$ 2$ per person, while the cost per user, in the intervention sub-sample was calculated as approximately $\$ 40$ per person. 


\section{Discussion}

To gauge the representativeness of both the general sample and the intervention subsample, demographic and health behavior data were compared to the data gathered in 2010 by the American College Health Association (ACHA) at WVU. This survey was conducted via email (using students' MIX accounts) during the 2010 academic year. The general sample and the intervention sub-sample appeared to be demographically slightly different, being constituted by a younger population. Although no major differences were found in other demographics, different results were shown in terms of the main dependent variables (Table 1, 2). Although the ACHA sample showed almost similar results in terms of people meeting the drinking guidelines, the percentages of students reporting to have met the dietary guidelines (4.7\%) and the guidelines for physical activity (moderate $18.6 \%$ and vigorous $29.2 \%$ ) were substantially smaller than those presented by the samples of this study (respectively 79\%, 40.3\%, 69.4\%) (Table 2).

The participants who volunteered in this study were likely to meet the guidelines for physical activity, nutrition, and diet as gathered by the survey, and showed a relatively high likelihood to engage in these behaviors in the future. These characteristics made this sample very similar to the sample considered by Newton at al. (2006) and to the wider sample considered by the ACHA survey in 2010. The healthy characteristics of these samples were confirmed by comparing them to other studies' samples. Only $1.7 \%$ of the intervention sub-sample and $2.9 \%$ of the general sample experienced heavy drinking in the previous month, while a higher percentage $(7.5 \%)$ was noted in the 2008 National Survey on Drug Use and Health (NSDUH; SAMHSA, 2008).

Another comparison concerns the dietary guidelines. The majority of the general $(72.6 \%)$ and intervention sub-sample (79\%) reported meeting the fruit and vegetables guidelines, while 
Racette et al. (2005) found that one third of their college students sample met this guideline and Anding et al. (2001) found that $43 \%$ of college women followed at least one of the dietarian guidelines. This substantially healthier nutrition could also have been influenced by the fact that the sample in this study was mostly living in the residential halls or at least recruited outside of the residential halls cafeteria, where fruit and vegetables are easily accessible. This consideration can be sustained also by the fact that around $50 \%$ of the study sample in all three administration, respectively $48.4 \%, 43.5 \%$, and $51.6 \%$ stated to have meals in the residence hall cafeteria 4 days out of 7 a week. This tendency to be healthier than the general population can be justified by the hypothesis that, generally, healthier or more conscientious individuals are more inclined to join health-related interventions (Buscemi, Murphy, \& Martens, 2010). However, these healthy attitudes and behaviors of the sample may have also created a ceiling effect, rendering both forms of the intervention less effective.

Despite this potential ceiling effect, the self-reported likelihood of these individuals to engage in all health behaviors increased slightly over the eight-week intervention. The greater effects might be due to the influence that online self-testing and the informative aspect of the intervention had on these individuals (Brunsting \& van den Putte, 2006; Soweid, Kak, El, Major, Karam, \& Rouhana, 2003). Regardless of the small size of these changes, all values generally showed a high consideration for the behaviors, inferring that participation might have reinforced their habits.

Comparing the two sub-samples, personalized and normative feedback, no major differences were found over time, thus the tailored intervention did not show the intended effect in most dependent measures. Both sub-samples showed small increases in their level of physical activity, whereas they presented a small decrease in their consumption of fruit and vegetables per 
day, and an increase in both alcoholic drinks per day and in days with at least one alcoholic drink. Differently than the normative feedback sample, the personalized feedback showed a small decrease in both moderate and heavy drinking. Interestingly, the individuals in this sample varied in their readiness to change in some of these behaviors, showing progression in both physical activity and drinking habits. In terms of PA, $62.9 \%$ of the intervention sub-sample stayed in the same stage of change, whereas $12.9 \%$ moved up in terms of stages. With nutrition, $54.8 \%$ stayed in the same stage and $20.9 \%$ moved up, whereas in terms of alcohol, $56.5 \%$ stayed in the same stage and $18.7 \%$ moved up to a different stage. Those individuals who moved up in terms of stages, might have increased their awareness, which brought them to be more inclined to think about the behavior, to prepare for it, or to be more engaged in it (Freemantle et al., 1993), while the higher percentage of people staying in the same stage, might be due to the ceiling effect showed by the intervention sample.

The potential public health effects of this and similar tailored interventions could be positive, even with small effects, when applied on a larger scale (Kroeze et al., 2006). The limited effects obtained could depend on many factors, such as the length and the structure of the intervention, or the nature and form of the offered feedback. This low effect of the intervention could also be due the fact that "only" $60 \%$ of this sample received and read the feedback. Interestingly, those who received the personalized feedback read it (80\%) and considered it useful $(77 \%)$.

Among this group of individuals, only a small percentage $(<10 \%)$ participated in the entire intervention. This small percentage, based on the obtained demographics, might have been the healthiest portion of the recruited sample, and therefore the most interested in a health-based intervention. 
This study confirms the low delivery costs of this type of intervention as sustained by Nigg (2003). Although the final cost per user in this study was approximately $\$ 40$, the low delivery cost for the overall survey sample ( $\$ 2$ per person) suggests that this intervention is an affordable approach with a potential for wide delivery. Despite the small affects incurred, if the intervention encourages participants to adopt more positive attitudes and behaviors, this approach could be easily justified and repeated with future groups of students. Some of the open comments shared by the participants also confirm that a prevention program that specifically addresses college students and is delivered through the Internet could be feasible and efficient, confirming results from previous work in the area (Moore, Soderquist, \& Werch, 2005; ScottSheldon, DeMartini, Carey, \& Carey, 2009).

\section{Limitations}

A major limitation of this study has been represented by the recruitment and by the maintenance of the initial sample. This difficulty is evident in the difference in participation between the fall and the spring administrations. The main difference between the two administrations was the presence of academic incentives; program compliance was found to be higher when the survey accounted for $5 \%$ of the participants' final grade (DeVahl, King, \& Williamson, 2005; Newton et al. 2006). The individuals who piloted the study in Spring 2010 also confirmed the potential beneficial effects of having the survey linked to a class structure, and, consequentially, providing an academic reward. As in Newton et al. (2006), this study was also unable to reach the entire incoming freshmen class, thus limiting external validity. Problems were also created by the self-selecting process of the participating sample, which resulted in a group of individuals already healthy or at least aware or curious about a healthier life style, excluding the part of the population that was initially thought as the target of the intervention. 
Another limitation could be related strictly to the survey in its length and content. The extensive length of the questionnaire could potentially have affected the participation of the students, as reported in some of the gathered open comments. In terms of the covered topics, while physical activity and nutrition are not generally considered "threatening" issues, alcohol use could have been seen as "threatening" because of the average age of the majority of the sample.

Additionally, some participants reported the inability of the feedback to vary according to accumulating gathered data. Some comment targeted the presence of identical questions in every administration and about the numerous repetitions of the survey. Other factors that have limited this study are those related to technical and software-related issues. Many difficulties were experienced during the preparation of the project and during the three administrations of the survey, such as connection or system problems. Due to an extensive delay of the delivery of the complete software, the study was moved from the Fall to the Spring semester. The major adjustment was characterized by the sample recruitment, which from a class-based sample moved to a voluntary sample. This effect can be potentially seen in the different percentage of people completing the survey at least once between the Fall $(64.55 \%)$ and the Spring administration $(16.21 \%)$.

\section{Conclusion and Future Directions}

Future studies may want to look at the possibility of increasing the level of the external validity in this type of intervention. With this goal in mind, future studies trying to address only freshmen and college students might want to consider establishing cooperation with the participating institution (which was attempted in this study, but declined by UNIV 101 coordinators). Future studies could access the email list of the entire freshmen class and provide 
this service as an integral part of the introductory classes, as suggested by DeVahl et al. (2005), or they could create a specific 1-credit class that incorporates the intervention as well as some educational modules focusing on these behaviors. An example of this potential class can be the PE 293 Physical Activity and Wellness for Life class offered at WVU for students who are on academic probation. An initial, introductory face-to-face presentation of the positive personal and academic effects of a healthy life could potentially increase the effectiveness of the intervention as well (Vandelanotte et al., 2007). Although in this study the sample gathered from the "in-class" recruiting process did not have a strong impact on the results, it could potentially have had an impact on the study based on the fact that the selected classes were mostly healthrelated major classes. These students might have had a tendency to be already interested in health or to be already engaged in a healthy lifestyle. This tendency could have created a self -selecting process that could have had some influenced on the results, not showing the expected results and to address a less needed population, while missing to address the originally targeted population.

To decrease the attrition and increase the external validity, these interventions could be provided as a "welcoming" service provided to all incoming freshman students. This strategy is embraced by WVU using AlcoholEdu, which is an alcohol-related confidential service provided online. The need of this sort of structured intervention has been sustained also by Carey, ScottSheldon, Carey, and DeMartini (2007) who found that alcohol risk reduction interventions are effective in influencing healthier drinking behaviors. Similar interventions could also be effective in addressing other heath behaviors. Using bigger incentives, incentives for everyone, leveled incentives, or "first come first serve" incentives (Koo \& Skinner, 2005), could be another strategy to increase the sample size and reduce attrition. Addressing more specifically the high attrition rate, more personalized emails, as recommended by Dillman (2007), might be useful, or 
if possible, having access to the participant phone contact, creating a system of reminders through automatic text messages. Embracing this delivery system could also favorite the increase of the economic benefits of this intervention. If addressing an entire freshman class, which consists of around 5,000 people, and made obligatory, both the delivery cost and the user cost would drop to a few cents per person. Specifically, if this intervention would be repeated with the WVU freshman class, this cost would be around $\$ 0.50$ per person.

Many studies already confirmed the effectiveness of KBS to deliver health behavior change interventions (i.e. Marcus et al., 2006; Spittaels et al., 2007; Vandelanotte et al., 2005), and, for this reason, future studies might want to work on more in depth and more structured feedback with a simpler survey format. These studies could also look at the use of alternative and integrated strategies, such as Experiential Sampling Methods, the use of text messaging, and social networks, to better capture the interest of this population. These studies could explore the effectiveness of the use of multiple contacts at lower "intensity", addressing different behaviors, increasing the interest of the participants and to strengthen the effect of the intervention, maintaining its low cost and wide appeal.

Future practitioners could also consider delivering printable or audio feedback, which may provide more concise feedback. As suggested by Noar et al. (2007), future research could be more careful in developing an even more personalized feedback, strategically addressing the specific level of readiness to change and the demographic characteristics of the individuals to a greater extent.

The preventive aspects of this type of intervention could provide institutions with a useful tool to supply helpful services to their students and, with small changes in the structure of the survey and of the intervention, eventually also to their faculty and staff. The preventive aspects, 
if validated, could also help institutions both limit retention problems caused by alcohol misuse (Thompson, 2007) and address the direct and indirect consequences of unhealthy diet or inactive lifestyle through the delivery of "curative" health services. 


\section{References}

(2002, September 16). Internet essential to college life, study says. Ascribe Newswire: Health, 3-4. Retrieved from Health Source - Consumer Edition database.

Ajzen, I. (1985). From intentions to actions: A theory of planned behavior. In J. Kuhl, \& J. Beckmann (Eds.), Springer series in social psychology (pp. 11-39). Berlin: Springer.

Ajzen, I. (1991). The theory of planned behavior. Organizational Behavior and Human Decision Processes, 50(2), 179-211.

Anderson, K. (2001). Internet use among college students: An exploratory study. Journal of American College Health, 50(1), 21-27.

Anding, J.D., Suminski, R.R., \& Boss, L. (2001). Dietary intake, body mass index, exercise, and alcohol: are college women following the dietary guidelines for Americans? Journal of American College Health, 49(4), 167-171.

Arnett, J.J. (2000). Emerging adulthood: A theory of development from the late teens through the twenties. American Psychologist, 55(5), p. 469-480.

Brunsting, S. \& van den Putte, B. (2006). Web-based computer-tailored feedback on alcohol use: Motivating excessive drinkers to consider their behavior. In: The Internet and health care: Theory, research, and practice. Murero, M. \& Rice, R.E. (Eds.); Mahwah, NJ, US: Lawrence Erlbaum Associates Publishers, 2006. pp. 313-333.

Buscemi, J., Murphy, J.G., Martens, M.P., McDevitt-Murphy, M.E., Dennhardt, A.A., Skidmore, J.R. (2010). Help-seeking for alcohol-related problems in college students: Correlates and preferred resources. Psychology of Addictive Behaviors, 24(4), 571-580

Carey, K.B., Scott-Sheldon, L.A.J., Carey, M.P., \& DeMartini, K.S. (2007). Individual-level interventions to reduce college student drinking: A meta-analytic review. Addictive 
Behaviors, 32(11), 2469-2494

Center for Disease Control and Prevention and Health Promotion. Behavioral Risk Factor Surveillance System (2007). Retrieved from http://apps.nccd.cdc.gov/BRFSS/display.asp?cat=FV\&yr=2007\&qkey=4415\&state=US

Cohen, J. (1988). Statistical power analysis for the behavioral sciences (2nd ed.). Hillsdale, NJ: Lawrence Earlbaum Associates.

DeVahl, J., King, R., Williamson, J.W. (2005). Academic incentives for students can increase participation in and effectiveness of a physical activity program. Journal of American College Health, 53(6), 295-298

Dillman, D.A. (2007). Mail and Internet Surveys. The tailored Design Method. (2 ${ }^{\text {nd }}$ Ed). John Wiley \& Sons, Inc. Hoboken, NJ

Engelmore, R.S. \& Feigenbaum, E. (1993). Expert Systems and artificial intelligence. In: Engelmore, R.S. (Ed.) Knowledge-Based Systems in Japan. Retrieved from: http://www.wtec.org/loyola/kb/toc.htm

Ferrara, C., Laurent, C. St., \& Wilson, T. (2008). The benefits of a weight loss contest in overweight and obese college students. Recreational Sports Journal, 32(1), 45-51.

Ferrara, C.M. (2009). The college experience: physical activity, nutrition, and implications for intervention and future research. Journal of Exercise Physiologyonline, 12(1), 23-35

Freemantle, N., Gill, P., Godfrey, C., Long, A., Richards, C., Sheldon, T.A., Song, F., Webb, J. (1993). Brief interventions and alcohol use. Quality in Health Care, 2, 267-273

Hargittai, E., \& Hinnant, A. (2008). Digital inequality: Differences in young adults' use of the internet. Communication Research, 35(5), 602-621.

Hull, H., Morrow, M., Heesch, K., Dinger, M., Han, J., \& Fields, D. (2007). Effect of the Summer 
Months on Body Weight and Composition in College Women. Journal of Women's Health, 16(10), 1510-1515.

Keating, X.D. Guan, J. Piñero, J.C., \& Bridges, D.M. (2005). A meta-analysis of college students' physical activity behaviors. Journal of American College Health, 54(2), 116-125.

Kelder, S.H., Perry, C.L., Klepp, K.I. \& Lytle, L.L. (1994). Longitudinal tracking of adolescent smoking, physical activity, and food choice behaviors. American Journal of Public Health, 84(7), 1121-1126

Koo, M. \& Skinner, H. (2005). Challenges of Internet recruitment: A case study with disappointing results. Journal of Medical Internet Research, 7(1), e6

Kreuter, M., Strecher, V., \& Glassman, B. (1999). One size does not fit all: The case for tailoring print materials. Annals of Behavioral Medicine, 21(4), 276-283.

Kroeze, W., Werkman, A., Brug, J. (2006). A systematic review of randomized trials on the effectiveness of computer-tailored education on physical activity and dietary behaviors. Annals of Behavioral Medicine, 31(3), 205-223.

Lien, N., Lytle, L.A., \& Klepp, K. (2001). Stability in consumption of fruit, vegetables, and sugary foods in a cohort from age 14 to age 21. Preventive Medicine, 33(3), 217-226

Lowry, R., Galuska, D.A., Fulton, J.E., Wechsler, H., Kann, L., \& Collins, J.L. (2000). Physical activity, food choice, and weight management goals and practices among US college students. American Journal of Preventive Medicine, 18(1), 18-27.

Lustria, M., Cortese, J., Noar, S., \& Glueckauf, R. (2009). Computer-tailored health interventions delivered over the web: Review and analysis of key components. Patient Education and Counseling, 74(2), 156-173. 
Marcus, B.H. Williams, D.M., Dubbert, P.M., Sallis, J.F., King, A.C., Yancey, A.K., Franklin, B.A., Buchner, D., Daniels, S.R., \& Claytor, R.P. (2006). Physical activity intervention studies. What we know and what we need to know. A scientific statement from the American Heart Association Council on Nutrition, Physical Activity, and Metabolism (Subcommittee on Physical Activity); Council on Cardiovascular Disease in the Young; and the Interdisciplinary Working Group on Quality of Care and Outcomes Research. Circulation, 114(24), 2739-2752

McCracken, M., Jiles, R., \& Blanck, H.M. (2007). Health behaviors of the young adult U.S. population: Behavioral Risk Factor Surveillance System, 2003. Preventing Chronic Disease: Public Health Research, Practice and Policy, 4(2), [11/7/09]. Available from: http://www.cdc.gov/pcd/issues/2007/apr/06_0090.htm

Medalie, J. (1981). The college years as a mini-life cycle: developmental tasks and adaptive options. College Health, 30(2), 75-79.

Mihalopoulos, N.L., Auinger, P., \& Klein, J.D. (2008). The Freshman 15: is it real? Journal of American College Health, 56(5), 531-533

Miller, K.H., Ogletree, R.J., \& Welshimer, K. (2002). Impact of activity behaviors on physical activity identity and self-efficacy. American Journal of Health Behavior, 26(5), 323-330

Moore, M.J., Soderquist, J., Werch, C. (2005). Feasibility and efficacy of a binge drinking prevention intervention for college students delivered via the Internet versus postal mail. Journal of American College Health, 54(1), 38-44

National Telecommunications and Information Administration. (2004). A nation online: Entering the broadband age. Washington, DC: U.S. Department of Commerce. 
Nelson, M.C., Story, M., Larson, N.I., Neumark-Sztainer, D., \& Lytle, L.A. (2008). Emerging adulthood and college-aged youth: an overlooked age for weight-related behavior change. Obesity, 16(10), 2205-2011

Newton, F., Kim, E., \& Newton, D. (2006). A program to establish healthy lifestyle behaviors with freshmen students. NASPA Journal (National Association of Student Personnel Administrators, Inc.), 43(3), 497-517.

Nigg, C. (2003). Technology's influence on physical activity and exercise science: The present and the future. Psychology of Sport and Exercise, 4(1), 57-65.

Noar, S.M., Benac, C.N., \& Harris, M.S. (2007). Does tailoring matter? Meta-analytic review of tailored print health behavior change interventions. Psychological Bulletin, 133(4), 673-693.

Park, M.J., Mulye, T.P., Adams, S.H., Brindis, C.D., Irwin, C.E. (2006). The health status of young adults in United States. Journal of Adolescent Health, 39(3), 305-317

Powell, J., Darvell, M., \& Gray, J. (2003). The doctor, the patient and the world-wide web: how the internet is changing healthcare. Journal of The Royal Society Of Medicine, 96(2), 74-76.

Prochaska, J.J., Nigg, C.R., Spring, B.V., Wayne, F., Prochaska, J.O. (2010) The benefits and challenges of multiple health behavior change in research and in practice. Preventive Medicine: An International Journal Devoted to Practice and Theory, 50(1-2), 26-29.

Prochaska, J.O. \& DiClemente, C.C. (1982). Trans-theoretical therapy - toward a more integrative model of change. Psychotherapy: Theory, Research and Practice, 19(3), 276-288.

Prochaska, J.O. \& DiClemente, C.C. (1983). Stages and processes of self-change of smoking: toward an integrative model of change. Journal of Consulting and Clinical Psychology, 51(3), 390395. 
Racette, R.B., Deusinger, S.S., Highstein, G.R., Strube, M.J., \& Deusinger, R.H. (2005). Weight changes, exercise, and dietary patterns during freshman and sophomore years of college. Journal of American College Health, 53(6), 245-251

Schröder, H., Morales-Molina, J.A., Bermejo, S., Barral, D., Mándoli Soler, E., Grau, M., Guxens, M., de Jaime Gil, E., Álvarez, Domínguez, M. Marrugat, J. (2007). Relationship of abdominal obesity with alcohol consumption at population scale. European Journal of Nutrition, 46(7), 369-376

Scott-Sheldon, L.A.J., Demartini, K.S., Carey, K.B., \& Carey, M.P. (2009). Alcohol interventions for college students improves antecedents of behavioral change: Results from a meta-analysis of 34 randomized controlled trials. Journal of Social and Clinical Psychology, 28(7), 799823

Soweid, R.A., Kak, A., El, F., Major, S.C. Karam, D.K., Rouhana, A. (2003). Changes in healthrelated attitude and self-reported behaviour of undergraduate students at the American University of Beirut following a health awareness course. Education for Health: Change in Learning \& Practice, 16(3), 265-278

Spittaels, H., De Bourdeaudhuij, I., Brug, J., \& Vandelanotte, C. (2007). Effectiveness of an online computer-tailored physical activity intervention in a real-life setting. Health Education Research, 22(3), 385-396.

Storer, J. H., Cychosz, C. M., \& Anderson, D. F. (1997). Wellness behaviors, social identities, and health promotion. American Journal of Health Behavior, 21(4), 260-268.

Strecher, V., Shiffman, S., \& West, R. (2005). Randomized controlled trial of a web-based computer-tailored smoking cessation program as a supplement to nicotine patch therapy. Addiction, 100(5), 682-688. 
Thompson, K.M. (2007). Alcohol-related legal infractions and student retention. Journal of Studies on Alcohol and Drugs, 68(5), 689-696

U.S. Department of Health and Human Services (2008). 2008 Physical activity guidelines for Americans. Washington, DC: U.S. Government Printing Office. Available at: http://www.health.gov/PAGuidelines/pdf/paguide.pdf. Accessed 04/15/09.

U.S. Department of Health and Human Services and U.S. Department of Agriculture (2005). Dietary guidelines for Americans, 2005. (6th Ed), Washington, DC: U.S. Government Printing Office, January 2005. Available at: http://www.health.gov/DietaryGuidelines/Accessed 11/21/09.

U.S. Department of Health and Human Services. Substance Abuse \& Mental Health Service Administration (2008). Results from the 2008 National Survey on Drug Use and Health: National Findings. Available at: http://www.oas.samhsa.gov/NSDUH/2k8NSDUH/tabs/TOC.htm Accessed 11/21/09.

Vandelanotte, C., Spathonis, K., Eakin, E., \& Owen, N. (2007). Website-delivered physical activity interventions: A review of the literature. American Journal of Preventive Medicine, 33(1), 54-64.

Weitzel, J., Bernhardt, J., Usdan, S., Mays, D., \& Glanz, K. (2007). Using wireless handheld computers and tailored text messaging to reduce negative consequence of drinking alcohol. Journal of Studies on Alcohol and Drugs, 68(4), 534-537. 
Table 1. Demographics of the three samples

\begin{tabular}{|c|c|c|c|c|c|c|}
\hline & \multicolumn{2}{|c|}{ ACHA $(\mathrm{N}=714)$} & \multicolumn{2}{|c|}{ General $(\mathrm{N}=303)$} & \multicolumn{2}{|c|}{ Intervention $(\mathrm{n}=62)$} \\
\hline & Mean & Std. Dev. & Mean & Std. Dev. & Mean & Std. Dev. \\
\hline Age & 23.38 & 4.80 & 18.97 & 2.05 & 19.39 & 1.71 \\
\hline Height & 67.42 & 4.04 & 67.45 & 7.50 & 66.74 & 4.14 \\
\hline Weight & 164 & 41.32 & 164.94 & 38.56 & 158.91 & 4014 \\
\hline BMI & 25.19 & 5.13 & 24.96 & 4.83 & 24.98 & 5.78 \\
\hline Female & \multicolumn{2}{|c|}{61.3} & \multicolumn{2}{|c|}{53.8} & \multicolumn{2}{|c|}{59.7} \\
\hline \multicolumn{7}{|l|}{ Age } \\
\hline $18-20$ years & \multicolumn{2}{|c|}{6.8} & \multicolumn{2}{|c|}{82.5} & \multicolumn{2}{|c|}{82.3} \\
\hline 21-24 years & \multicolumn{2}{|c|}{75.6} & \multicolumn{2}{|c|}{10.6} & \multicolumn{2}{|c|}{16.1} \\
\hline 25 years or older & \multicolumn{2}{|c|}{17.7} & \multicolumn{2}{|c|}{6.9} & \multicolumn{2}{|c|}{1.6} \\
\hline \multicolumn{7}{|l|}{ Race } \\
\hline White & \multicolumn{2}{|c|}{92.6} & \multicolumn{2}{|c|}{88.2} & \multicolumn{2}{|c|}{95.2} \\
\hline Black & \multicolumn{2}{|c|}{2.1} & \multicolumn{2}{|c|}{3.5} & \multicolumn{2}{|c|}{1.6} \\
\hline Hispanic & \multicolumn{2}{|c|}{1.5} & \multicolumn{2}{|c|}{1} & \multicolumn{2}{|c|}{0} \\
\hline Asian & \multicolumn{2}{|c|}{2.4} & & & & \\
\hline Native Americans & & & & & & \\
\hline Multi \& Other & & & & & & 6 \\
\hline College Class & & & & & & \\
\hline Freshmen & & & & & & \\
\hline Sophomore & & & & & & .6 \\
\hline Junior & & & & & & \\
\hline Senior & & & & & & \\
\hline Living & & & & & & \\
\hline Dorms & & & & & & \\
\hline Off Campus & & & & & & \\
\hline Univ. Prov. & & & & & & \\
\hline At Home & & & & & & \\
\hline BMI & & & & & & \\
\hline Underweight & & & & & & 6 \\
\hline Normal & & & & & & 9 \\
\hline Overweight & & & & & & 3 \\
\hline Obese & & & & & & .4 \\
\hline Life Style & & & & & & \\
\hline Very Healthy & & & & & & .5 \\
\hline Healthy & & & & & & .8 \\
\hline Unhealthy/very & & & & & & 6 \\
\hline
\end{tabular}

Note: The used assessment in the two main samples were not exactly the same and for this reason, the data have been elaborated in order to be as comparable as possible

- Age: the used categories for this table were those used in the ACHA survey, the answers provided by the study sample have been re-grouped in different categories

- Life Style: the options for answering were different. Excellent + Very Good = Very Healthy; Good + Fair = Healthy; Poor = Unhealthy + Very Unhealthy

- In term of the guidelines the answers in the study sample have been regrouped to match the ACHA options 
Table 2. Percentage of Individuals meeting guidelines of health behaviors in the three samples

\begin{tabular}{lccccccccc}
\hline \multicolumn{3}{c}{ ACHA Sample $(\mathrm{N}=714)$} & \multicolumn{3}{c}{ General sample $(\mathrm{N}=303)$} & \multicolumn{3}{c}{$\begin{array}{c}\text { Intervention Sample } \\
(\mathrm{n}=62)\end{array}$} \\
\hline Guidelines & $\begin{array}{c}\text { Male } \\
(\%)\end{array}$ & $\begin{array}{c}\text { Female } \\
(\%)\end{array}$ & $\begin{array}{c}\text { Total } \\
(\%)\end{array}$ & $\begin{array}{c}\text { Male } \\
(\%)\end{array}$ & $\begin{array}{c}\text { Female } \\
(\%)\end{array}$ & $\begin{array}{c}\text { Total } \\
(\%)\end{array}$ & $\begin{array}{c}\text { Male } \\
(\%)\end{array}$ & $\begin{array}{c}\text { Female } \\
(\%)\end{array}$ & $\begin{array}{c}\text { Total } \\
(\%)\end{array}$ \\
\hline Moderate PA & 23 & 16.1 & 18.6 & 50.3 & 51.1 & 50.5 & 36 & 43.2 & 40.3 \\
Vigorous PA & 31.2 & 28.4 & 29.2 & 63.2 & 59 & 61.1 & 68 & 70.3 & 69.4 \\
Combined PA & 53.4 & 45 & 47.8 & 88.9 & 83.9 & 86.3 & 76 & 83.8 & 80.6 \\
Servings F\&V & 7.2 & 3 & 4.7 & 71.2 & 74.8 & 72.6 & 80 & 78.4 & 79 \\
Al Moderate & N/A & N/A & N/A & 56.4 & 66.2 & 61.1 & 84 & 67.6 & 74.2 \\
Al Heavy & 72.4 & 83.5 & 79.4 & 68.1 & 83.5 & 75.2 & 92 & 97.3 & 95.2 \\
\hline
\end{tabular}

Variables:

- Moderate PA: Moderate Physical Activity

- Vigorous PA: Vigorous Physical Activity

- Combined PA: Sum of Moderate and Vigorous Physical Activity

- Serving F \& V: Servings of Fruit and Vegetables

- Al Moderate: Moderate Drinking

- Al Heavy: Heavy Drinking 
Table 3. Descriptive data related to the Theory of Planned Behavior, the TTM, and the health behavioral habits of the WVU samples, divided by General, Spring and Intervention Sample

\begin{tabular}{|c|c|c|c|c|c|c|}
\hline & \multicolumn{2}{|c|}{ General $(n=303)$} & \multicolumn{2}{|c|}{ Spring $(n=149)$} & \multicolumn{2}{|c|}{ Intervention $(\mathrm{n}=62)$} \\
\hline Female & \multicolumn{2}{|c|}{53.8} & \multicolumn{2}{|c|}{61.3} & \multicolumn{2}{|c|}{59.7} \\
\hline Moderate PA & \multicolumn{2}{|c|}{50.5} & \multicolumn{2}{|c|}{47.7} & \multicolumn{2}{|c|}{40.3} \\
\hline Vigorous PA & \multicolumn{2}{|c|}{61.1} & \multicolumn{2}{|c|}{58.4} & \multicolumn{2}{|c|}{69.4} \\
\hline Servings F\&V & \multicolumn{2}{|c|}{72.6} & \multicolumn{2}{|c|}{69.1} & \multicolumn{2}{|c|}{80.6} \\
\hline Al Moderate & \multicolumn{2}{|c|}{61.1} & \multicolumn{2}{|c|}{59.1} & \multicolumn{2}{|c|}{74.2} \\
\hline \multirow[t]{3}{*}{ Al Heavy } & & & & & & \\
\hline & \multicolumn{2}{|c|}{ General $(n=303)$} & \multicolumn{2}{|c|}{ Once Spring $(n=149)$} & \multicolumn{2}{|c|}{ Intervention $(\mathrm{n}=62)$} \\
\hline & Mean & $\underline{\mathrm{SD}}$ & Mean & $\underline{\mathrm{SD}}$ & Mean & $\underline{\mathrm{SD}}$ \\
\hline \multicolumn{7}{|l|}{$\mathrm{PA}$} \\
\hline Likelihood & 4.89 & 1.34 & 4.84 & 1.29 & 4.84 & 1.57 \\
\hline Importance & 5.18 & 1.03 & 5.17 & 0.97 & 5.24 & 0.84 \\
\hline \multicolumn{7}{|l|}{ NU } \\
\hline Likelihood & 4.60 & 1.29 & 4.67 & 1.22 & 4.77 & 1.12 \\
\hline Importance & 4.60 & 1.22 & 4.97 & 1.10 & 5.08 & 0.99 \\
\hline \multicolumn{7}{|l|}{$\mathrm{Al}$} \\
\hline Likelihood & 3.71 & 1.91 & 3.82 & 1.85 & 4.13 & 1.91 \\
\hline \multirow[t]{3}{*}{ Importance } & 3.78 & 1.98 & 4.01 & 1.84 & 4.46 & 1.48 \\
\hline & \multicolumn{2}{|c|}{ General $(n=303)$} & No Tre & $=149)$ & Interve & $n=62)$ \\
\hline & & & & & & \\
\hline PA & & & & & & \\
\hline Precontemplation & & & & & & \\
\hline Contemplation & & & & & & \\
\hline Preparation & & & & & & \\
\hline Action & & & & & & \\
\hline Maintenance & & & & & & \\
\hline NU & & & & & & \\
\hline Precontemplation & & & & & & \\
\hline Contemplation & & & & & & \\
\hline Preparation & & & & & & \\
\hline Action & & & & & & \\
\hline Maintenance & & & & & & \\
\hline $\mathrm{Al}$ & & & & & & \\
\hline Precontemplation & & & & & & \\
\hline Contemplation & & & & & & \\
\hline Preparation & & & & & & \\
\hline Action & & & & & & \\
\hline Maintenance & & & & & & \\
\hline
\end{tabular}

Variables:

- PA, NU, AL: Physical Activity, Nutrition, Alcohol

- Moderate PA \& Vigorous PA: Moderate Physical Activity \& Vigorous Physical Activity

- Serving F \& V: Servings of Fruit and Vegetables

- Al Moderate \& Al Heavy: Moderate Drinking \& Heavy Drinking

- Likelihood and Importance: Likelihood to engage in the behaviors and importance attributed to the behavior 
Table 4. Comparison of health behaviors and TBP variables reports between the two feedbacks, along the 3 administrations

\begin{tabular}{|c|c|c|c|c|c|c|c|c|c|c|c|c|}
\hline & \multicolumn{4}{|c|}{ Time 1} & \multicolumn{4}{|c|}{ Time 2} & \multicolumn{4}{|c|}{ Time 3} \\
\hline & \multicolumn{2}{|c|}{ Personalized } & \multicolumn{2}{|c|}{ Normative } & \multicolumn{2}{|c|}{ Personalized } & \multicolumn{2}{|c|}{ Normative } & \multicolumn{2}{|c|}{ Personalized } & \multicolumn{2}{|c|}{ Normative } \\
\hline & Mean & $\underline{\mathrm{SD}}$ & Mean & $\underline{\mathrm{SD}}$ & $\underline{\text { Mean }}$ & $\underline{\mathrm{SD}}$ & $\underline{\text { Mean }}$ & $\underline{\mathrm{SD}}$ & Mean & $\underline{\mathrm{SD}}$ & $\underline{\text { Mean }}$ & $\underline{\mathrm{SD}}$ \\
\hline Moderate PA & 3.87 & 1.84 & 4.26 & 1.89 & 3.39 & 1.94 & 4.87 & 1.86 & 4.10 & 1.58 & 4.65 & 1.76 \\
\hline Vigorous PA & 2.74 & 1.71 & 3.71 & 2.12 & 2.94 & 1.93 & 4.03 & 1.89 & 2.81 & 1.56 & 3.81 & 1.74 \\
\hline Servings F\&V & 7.42 & 3.63 & 7.39 & 2.98 & 6.97 & 4.63 & 7.81 & 3.24 & 6.90 & 3.42 & 7.06 & 3.14 \\
\hline Days w/1 drink & 0.97 & 1.19 & 0.9 & 1.35 & 1.19 & 1.28 & 0.97 & 1.33 & 1.10 & 1.37 & 1.19 & 1.38 \\
\hline Drinks per day & 0.70 & 1.05 & 0.7 & 1.15 & 1.03 & 1.47 & 0.84 & 1.42 & 0.77 & 1.38 & 0.84 & 1.32 \\
\hline Binge /Week & 0.57 & 0.97 & 0.63 & 1.13 & 0.68 & 1.08 & 0.55 & 1.06 & 0.55 & 1.26 & 0.84 & 1.32 \\
\hline \multirow[t]{4}{*}{ Binge /Month } & 0.90 & 1.12 & 0.77 & 0.97 & 1 & 1.03 & 0.84 & 1.13 & 0.65 & 0.71 & 0.87 & 0.99 \\
\hline & \multicolumn{4}{|c|}{ Time 1} & \multicolumn{4}{|c|}{ Time 2} & \multicolumn{4}{|c|}{ Time 3} \\
\hline & \multicolumn{2}{|c|}{ Personalized } & \multicolumn{2}{|c|}{ Normative } & \multicolumn{2}{|c|}{ Personalized } & \multicolumn{2}{|c|}{ Normative } & \multicolumn{2}{|c|}{ Personalized } & \multicolumn{2}{|c|}{ Normative } \\
\hline & $\underline{\text { Mean }}$ & $\underline{\mathrm{SD}}$ & $\underline{\text { Mean }}$ & $\underline{\mathrm{SD}}$ & $\underline{\text { Mean }}$ & $\underline{\mathrm{SD}}$ & $\underline{\text { Mean }}$ & $\underline{\mathrm{SD}}$ & $\underline{\text { Mean }}$ & $\underline{\mathrm{SD}}$ & $\underline{\text { Mean }}$ & $\underline{\mathrm{SD}}$ \\
\hline \multicolumn{13}{|l|}{ Physical Activity } \\
\hline Likelihood & 4.48 & 1.69 & 5.19 & 1.38 & 4.61 & 1.45 & 5.19 & 1.47 & 4.84 & 1.241 & 5.03 & 1.43 \\
\hline Importance & 5.10 & 0.83 & 5.39 & 0.84 & 5.10 & 0.91 & 5.35 & 0.88 & 5.06 & 0.964 & 5.26 & 1.15 \\
\hline \multicolumn{13}{|l|}{ Nutrition } \\
\hline Likelihood & 4.71 & 0.94 & 4.84 & 1.29 & 4.84 & 1.09 & 4.97 & 1.17 & 4.74 & 0.965 & 4.94 & 1.26 \\
\hline Importance & 5.06 & 0.89 & 5.10 & 1.11 & 5.13 & 0.81 & 5.29 & 1.01 & 5.06 & 0.929 & 5.23 & 0.99 \\
\hline \multicolumn{13}{|l|}{ Alcohol } \\
\hline Likelihood & 4.14 & 1.9 & 4.12 & 1.97 & 4.17 & 1.93 & 4.07 & 2.03 & 4.93 & 1.580 & 3.80 & 2.07 \\
\hline Importance & 4.64 & 1.52 & 4.27 & 1.84 & 3.89 & 1.97 & 4.07 & 1.87 & 4.69 & 1.514 & 4.03 & 1.90 \\
\hline
\end{tabular}

Variables:

- Moderate PA \& Vigorous PA: Moderate Physical Activity \& Vigorous Physical Activity

- Serving F \& V: Servings of Fruit and Vegetables

- Days w/1 drink \& Drinks per Day: Days with at least 1 drink \& the Number of drinks in a day

- Binge /Week and Binge /Month: Number of binge drinking episodes in a week or in a month

- Likelihood and Importance: Likelihood to engage in the behaviors and importance attributed to the behavior 
Table 5. Comparison of the data related to the health behaviors, the TPB of the complete sample participating in the study, looking at the time points.

\begin{tabular}{|c|c|c|c|c|c|c|}
\hline & \multicolumn{2}{|c|}{ Time 1} & \multicolumn{2}{|c|}{ Time 2} & \multicolumn{2}{|c|}{ Time 3} \\
\hline & Mean & $\underline{\mathrm{SD}}$ & Mean & $\underline{\mathrm{SD}}$ & Mean & $\underline{\mathrm{SD}}$ \\
\hline Moderate PA & 4.06 & 1.863 & 4.13 & 2.028 & 4.37 & 1.681 \\
\hline Vigorous PA & 3.23 & 1.970 & 3.48 & 1.973 & 3.31 & 1.714 \\
\hline Servings F\&V & 7.40 & 3.396 & 7.39 & 3.423 & 6.98 & 3.257 \\
\hline Days w/1 drink & 0.93 & 1.260 & 1.08 & 1.297 & 1.15 & 1.365 \\
\hline Drinks per day & 0.70 & 1.094 & 0.93 & 1.436 & 1.00 & 1.0547 \\
\hline Binge /Week & 0.60 & 1.045 & 0.61 & 1.061 & 0.69 & 1.288 \\
\hline \multirow[t]{3}{*}{ Binge /Month } & 0.83 & 1.044 & 0.92 & 1.076 & 0.76 & 0.862 \\
\hline & \multicolumn{2}{|c|}{ Time 1} & \multicolumn{2}{|c|}{ Time 2} & \multicolumn{2}{|c|}{ Time 3} \\
\hline & Mean & $\underline{\mathrm{SD}}$ & Mean & $\underline{\mathrm{SD}}$ & Mean & $\underline{\mathrm{SD}}$ \\
\hline PA & & & & & & \\
\hline Likelihood & 4.84 & 1.570 & 4.90 & 1.479 & 4.94 & 1.329 \\
\hline $\begin{array}{l}\text { Importance } \\
\text { NU }\end{array}$ & 5.24 & 0.843 & 5.23 & 0.895 & 5.16 & 1.059 \\
\hline Likelihood & 4.77 & 1.122 & 4.90 & 1.127 & 4.84 & 1.119 \\
\hline $\begin{array}{l}\text { Importance } \\
\mathrm{Al}\end{array}$ & 5.08 & 0.997 & 5.21 & 0.908 & 5.15 & 0.956 \\
\hline Likelihood & 4.13 & 1.914 & 4.12 & 1.966 & 4.36 & 1.919 \\
\hline Importance & 4.46 & 1.679 & 3.98 & 1.904 & 4.36 & 1.740 \\
\hline
\end{tabular}

Variables:

- Moderate PA \& Vigorous PA: Moderate Physical Activity \& Vigorous Physical Activity

- Serving F \& V: Servings of Fruit and Vegetables

- Days w/1 drink \& Drinks per Day: Days with at least 1 drink \& the Number of drinks in a day

- Binge /Week and Binge /Month: Number of binge drinking episodes in a week or in a month

- Likelihood and Importance: Likelihood to engage in the behaviors and importance attributed to the behavior 
Table 6. Comparison of the data related to the health behavior and the TTM of the complete sample participating in the study, looking at the time points.

\begin{tabular}{lccc}
\hline & Time 1 & Time 2 & Time 3 \\
\hline PA & $\%$ & $\%$ & $\%$ \\
Precontemplation & 3.2 & & \\
Contemplation & 8.1 & 1.6 & 4.8 \\
Preparation & 24.2 & 6.5 & 4.8 \\
Action & 19.4 & 22.6 & 30.6 \\
Maintenance & 45.2 & 45.2 & 17.7 \\
NU & & 45.2 & 41.9 \\
Precontemplation & 1.6 & 0 & 0 \\
Contemplation & 14.8 & 11.3 & 12.9 \\
Preparation & 52.5 & 45.2 & 48.4 \\
Action & 11.5 & 19.4 & 9.7 \\
Maintenance & 19.7 & 24.2 & 29 \\
Al & & & \\
Precontemplation & 14 & 18.6 & 21.3 \\
Contemplation & 7 & 6.8 & 4.9 \\
Preparation & 24.6 & 23.7 & 23 \\
Action & 5.3 & 5.1 & 1.6 \\
Maintenance & 49 & 45.8 & 49.2 \\
\hline
\end{tabular}

Variables:

- Moderate PA \& Vigorous PA: Moderate Physical Activity \& Vigorous Physical Activity

- Serving F \& V: Servings of Fruit and Vegetables

- Days w/1 drink \& Drinks per Day: Days with at least 1 drink \& the Number of drinks in a day

- Binge /Week and Binge /Month: Number of binge drinking episodes in a week or in a month

- Likelihood and Importance: Likelihood to engage in the behaviors and importance attributed to the behavior 
Figure1: Sampling/Recruitment Flow Chart

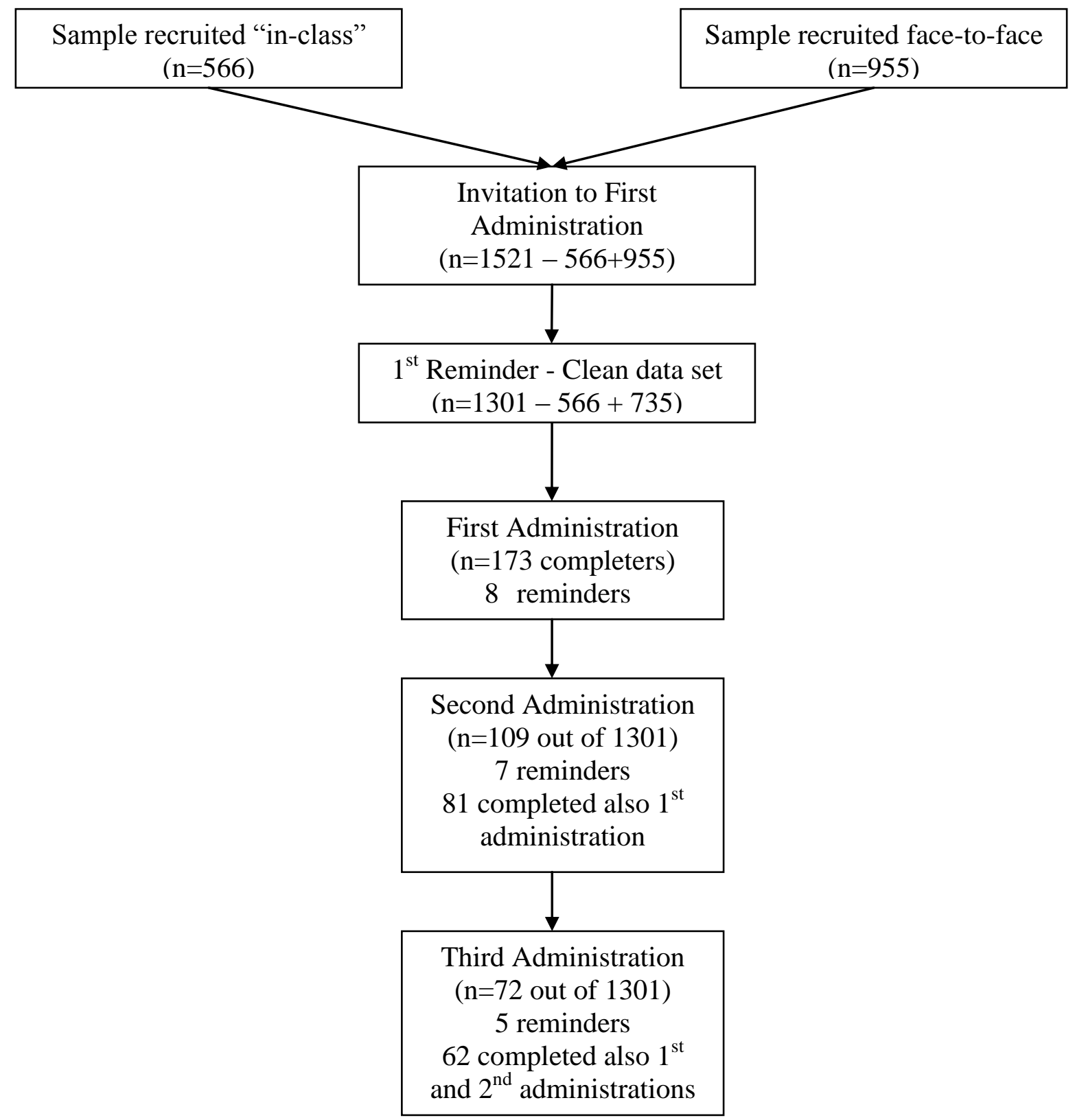


APPENDIX A: Review of Literature

\section{Introduction}

The intent of this review of literature is to explore the health behaviors and current health related situation of college students, who, once considered a healthy and low risk population, have recently been found to not be immune to unhealthy conditions. This population showed tht it is not invulnerable from weight gain, obesity, and from the development of many other unhealthy behaviors (i.e. physical inactivity, poor diet, and alcohol misuse) that can have, beside the direct immediate effects, implications on the their long-term life (Lien, Lytle, Klepp, 2001; Kvaavik, Andersen, \& Klepp, 2005; Sparling \& Snow, 2002). Recently, this population became the target of studies that have weight gain, obesity, and lack of healthy behaviors as their major goal. Many of these studies tried to develop and maintain interventions intended to increase these health behaviors and to diminish the detrimental effects of those unhealthy conditions affecting the well-being of the college student population.

Gordon-Larsen, Adair, Nelson, and Popkin (2004) found that most of the individuals that are obese in adolescence and young adulthood remained obese in adulthood, concluding that this period of life is potentially dangerous in terms of obesity development. While studies showed the stability of health related behaviors, such as some nutritional behaviors and habits, during the transition from adolescence to young adulthood and adulthood (i.e. Lien et al., 2001; Kvaavik et al., 2005), Sallis, Prochaska, and Taylor (2000) described how a decline in physical activity engagement and an increase in the levels of obesity and overweight were potential characteristics of this transition. Many studies found some of these developmental changes in the quantity and quality of some healthy behaviors, such as the decrease of the engagement in physical activity in adulthood (i.e. Zick, Smith, Brown, Fan, \& Kowaleski-Jones, 2007; Caspersen, Pereira, \& 
Curran, 2000). The decrease of the quality of the daily nutrition, such as the increase of fast food consumption and breakfast skipping (Niemeier, Raynor, Lloyd-Richardson, Rogers, \& Wing, 2006) was found as due to many different factors such as time and family issues (i.e. Caspersen et al.; Demory-Luce, Morales, Nicklas, Baranowski, Zacheri, \& Barenson, 2004). It has also previously shown how alcohol use is present across different age and with a natural increase, due to the age-law, toward adulthood.

McCracken, Jiles, and Blanck, (2007) sustained that health-risks are generally established during adolescence and young adulthood, and then maintained during adulthood. McTigue, Garrett, and Popkin (2002) addressed the necessity of an early intervention, noticing in their national sample, constituted by 9179 , how $80 \%$ of the obese adults developed this health condition during early adulthood. Based on similar findings, many other authors (i.e. Caspersent et al., 2000; Ferreira, Twisk, van Mechelen, Kemper, \& Stehouwer, 2005) highlighted the necessity of early interventions (e.g., during the transition from adolescence to adulthood) as a preventive tool against chronic diseases, promoting better nutrition, and increasing physical activity in order to control the individuals' weight.

This review is organized into the following different sections: a) the concept of health, b) the definition of the general problem, c) the emerging adulthood, d) the college life, e) the relationship between the college life and an healthy lifestyle, f) Intervention targeting healthy behaviors, g) mediated communication and its use in promoting healthy behaviors, h) the need of further research.

\section{The Concept of Health}

According to Dugdill, Crone, and Murphy (2009) health is a multidisciplinary concept. The World Health Organization (1948) defines health as "a state of complete physical, mental 
and social well-being and not merely the absence of disease or infirmity". These different aspects that constituted the concept of health allow looking at this concept from a holistic perspective, not limiting human beings to just one aspect, and all of them are strongly interrelated to each other. Physical and mental health are the most discussed aspects of health, but emotional, social and spiritual dimensions are relevant parts of the general health status as well (Dugdill et al., 2009).

Physical activity, diet, and alcohol use/misuse are three of the main behaviors/factors characterizing the individual lifestyle (it could be possible to add many others, including smoking, unsafe sexual behaviors, use of recreational drugs, etc), with potential consequences related to the quality of life for the adult population. Paying attention to these three behaviors and trying to improve and correct them can be considered an effective method to develop a healthy lifestyle. These three behaviors, however, are often misunderstood and can be perceived as difficult to integrate into a normal daily life.

\section{The Definition of the General Problem}

Lack of a healthy life style can easily lead to the development of unhealthy conditions such as obesity and being overweight. These are topics of global interest since their existence is a phenomenon that goes across countries (Wang, Monteiro, \& Popkin, 2002; Silventoinen, Sans, Tolonen, Monterde, Kuulasmaa, Kesteloot, \& Tuomilehto, 2004). With numbers rising to epidemic proportions, over a billion adults overweight, among whom more than 300 million are clinically obese (WHO, 2003).

Excess body weight is considered by the World Health Organization - Europe to be responsible for more than 1 million deaths and the loss of 12 million disability-adjusted lifeyears (DALYs) in Europe every year (WHO-Europe, 2007). According to the World Health 
Organization, similar situations are present all around the globe where the range of health-related consequences due to overweight and obesity goes from the increased possibility of premature mortality to a significant reduction of the quality of life due to the presence of serious chronic conditions and disability (WHO, 2003). Being obese and overweight has been found directly and indirectly associated to many different physical health issues, such as cardiovascular disease, type II diabetes, functional disability, musculo-skeletal disorders, hypertension and stroke, and certain forms of cancer (Anderson, Quinn, Glanz, Ramirez, Kahwati, Johnson, et al., 2009; Lewis, Jacobs, McCreath, Kiefe, Schreiner, Smith, \& Williams, 2000; Mokdad, Serdula, Dietz, Bowan, Marks, \& Koplan, 1999; Silventoinen et al., 2004; WHO, 2003), as well as some psychological and emotional distress, such as decreases in self-esteem and quality of life. In the US, it has been estimated that obesity-related health situations are the cause of 300,000 adult deaths and diabetes is the sixth leading cause of death; consequentially it has been found that obesity and diabetes, together, generate immense health care costs (Mokdad, Ford, Bowman, Dietz, Vinicor, Bales, et al., 2003; Mokdad, Marks, Stroup, \& Gerberding, 2004). Researchers have found that many of the leading causes of death in the US population are directly related to poor nutrition (Abood, Black, \& Feral, 2003) and that dietary factors can limit the development of illnesses and premature deaths (Plotnikoff, McCargar, Wilson, \& Loucaides, 2005). The great increase in the obesity level is clearly shown by the data representing how, according to the CDC data (2005), the level of obesity reached the $15 \%$ only in four states. In 2000 , only one state did not reach the level of $15 \%$ obesity or greater with 22 states reporting more than $20 \%$ of this prevalence. According to Ogden, Carroll, Curtin, Lamb, \& Flegal (2010) more than $34.2 \%$ of the children and adolescents (between 6 and 19 years old) are obese (over the 85 th percentile of BMI) and the $33.9 \%$ of the over 20 adult population is overweight or obese 
(Flegal, Carroll, Ogden, \& Curtin, 2010). Many recent estimates showed how most of the US states have experienced an increase in their level of overweight and obesity within their populations (Trust for America's Health, 2008; 2009), making the entire US population being characterized by the fact that, based on body mass index, more than $50 \%$ of the adult (18 or over) is overweight or obese, respectively $35 \%$ and $27 \%$ (CDC, 2008). This public health condition is present across different ages, genders (Wang, Beydoun, Liang, Caballero, \& Kumnyika, 2008; Odgen et al., 2006; McCracken, Jiles, \& Blanck, 2007), and ethnic groups (Gordon-Larsen, Adair, Nelson, \& Popkin, 2004). According to the CDC data report (2009), overweight men were more than women ( $42 \%$ vs. $28 \%$ ), whereas obesity levels were similar between men and women (29\% vs. $31 \%)$.

These consequences of being obese and overweight are not only limited to the single individual's life (i.e. shorter life expectancy), but they also have a major impact on the entire society. It is due to the fact that this condition increases the direct and indirect health related costs, such as diagnostic-related and costs related to morbidity and mortality. According to their data, these authors made some projections about a dramatic increase of the level of obesity within the U.S. population by 2030 characterized by an increase of obesity and overweight in adults $(0.77)$, and by women increasing faster than men (0.91 vs. 0.65$)$. More recent data, however, shows a more optimistic view based on the fact that the prevalence of obesity among women hit a plateau during the period from 1999 through 2008, while men stopped this increase between 2003 and 2008 (Neighmond, 2010). Despite these more optimistic projections, the public costs of obesity are still increasingly becoming public concern.

The development of many different behaviors has led to a significant increase in obesity rates (WHO, 2003; Anderson et al., 2009). Among these many behaviors, some have been 
recognized as primarily involved in sustaining the development of this unhealthy condition and the most relevant in terms of obesity development. Examples of these behaviors are the increased of the total daily energy intake (Silventoinen et al., 2004) and more specifically the increased consumption of more energy-dense, nutrient-poor foods, saturated fats (WHO, 2003), highsweetened food (Kvaavik, Andersen, \& Klepp, 2005) and of the use of alcohol (Nelson, Lust, Story, \& Ehlinger, 2009). Other important factors to be considered are the reduced amount physical activity and the increased amount of sedentary behaviors (Gordon-Larsen, Nelson, \& Popkin, 2004). These behaviors could be easily summarized in physical activity, nutrition, and drinking behaviors sustained by Croezen, Visscher, ter Bogt, Veling, and Haveman-Nies (2009) to be strongly linked to obesity development. They will be the targeted behaviors in the development of this review of literature. This review will not address the specifics of the physiological aspects of these behaviors or their consequences. It will focus on why these behaviors need to be modified in order to reach a better healthy lifestyle and how this change could be helped by interventions provided by institutions already in existence, such as academic institutions. It also focuses on how these behaviors could be maintained with the goal of implementing the general health during adulthood of this college student population.

Being overweight or obese is the result of a lack of balance between calories consumed through food and drinks, and calories used in the daily life. Starting form this concept, Sanchez, Norman, Sallis, Calfas, Cella, and Patrick (2007) sustained that improving behaviors currently present and associated with morbidity and mortality (such as physical activity, sedentary, diet behaviors, and alcohol use) in all-age populations, is a national health priority to improve the health of the current and future U.S. population (CDC, 2007; 2009; Wang et al., 2008). 


\section{The Current Situation Characterizing the U.S. Population Regarding Physical Activity,} Nutrition, and Alcohol Use

According to the CDC data (2009), the current U.S. population is characterized by $36 \%$ of inactive adult population, $31 \%$ with some moderate physical activity, and $33 \%$ engaged in moderate physical activity three or more times per week. More than half (59\%) of the adults population, 18 years of age and over has never engaged in any vigorous physical activity lasting 10 minutes or more per week, and only $26 \%$ did it three or more times per week as recommend by the U.S. Department of Health and Human Services (HHS, 2008). According to the CDC data (2009), women are more inactive than men; men instead engaged in moderate physical activity on a regular basis more often. More than half of the men, and more than $60 \%$ of women, within the adult population, have never engaged in vigorous physical activity lasting 10 minutes or more per week. Men seem to be slightly more consistent in engaging in Physically Activity three or more times a week than women, respectively $29 \%$ and $23 \%$.

The CDC data (2009) also showed how $50 \%$ of the U.S. adult population was characterized by regular drinkers (1-2 units/day without exceeding 14 units/week for men and 1 unit/day without exceeding 7units/week - as suggested by the Dietary Guidelines for Americans, 2005). This population was also characterized by $14 \%$ of infrequent drinkers, by $6 \%$ of former regular drinkers, by $9 \%$ of former infrequent drinkers, and by $21 \%$ of people who have never consumed alcohol. This population was also characterized by the higher percentage of regular male drinkers and former regular drinkers than female, which instead tended to be populated by more current or former infrequent drinkers or lifetime abstainers.

In terms of nutrition, according to the data recollected by the CDC (2007), only $24.3 \%$ of the entire U.S. adult population eats five or more servings of fruits or vegetables per day as 
recommended by the Dietary Guidelines for Americans published by the US Department of Health and Human Services (HHS) and the Department of Agriculture (USDA). Along with these negative aspects of the diet of the American population, time constriction, convenience, lifestyle, fast food, and other unhealthy nutritional habits, become a progressively more and more important part of the diet of the U.S. population (Paereatakul, Ferdinand, Champagne, Ryan, \& Bray, 2003).

\section{A Brief Picture of the Three Targeted Behaviors}

\section{Physical activity \& exercise}

Physical Activity (PA) is a complex multi-dimensional behavior describable in terms of frequency, duration, intensity and mode (Miles, 2007). This behavior has been defined by many different authors and in many different ways, but in terms of this literature review it will be considered the definition offered by the Department of Health and Human Services. The "2008 Physical Activity Guidelines for Americans", define physical activity more generally as any "bodily movement that enhances health".

All the potential positive consequences of PA can be associated not only to the health of the individual, but also to the health of the entire population (Haskell, Lee, Pate, Powell, Blair, Franklin, et al., 2007). The various dimensions of PA may have different health outcomes, and it is not very clear which of the different dimensions of PA has the main effect (Miles, 2007). In 2008, the U.S. Department of Health and Human Services published updated physical activity guidelines. According to these guidelines for substantial health benefits, adults should do at least 2 hours and 30 minutes a week of moderate-intensity aerobic physical activity, 1 hour and 15 minutes a week of vigorous-intensity aerobic physical activity or an equivalent combination of the two, preferably spread throughout the week and performed in episodes of at least 10-15 
minutes. Instead for more extensive health benefits, they should increase their aerobic PA to 5 hours a week of moderate intensity, or 2 and half hours a week of vigorous intensity aerobic physical activity, or an equivalent combination. Muscle-strengthening activities in a moderate or high intensity of all major muscle groups, 2 or more days a week, provide additional health benefits (USDHHS, 2008). What characterized these guidelines is the fact that they stressed that these suggestions need not to be executed all in one session, but each individual can follow them executing those behaviors in bouts of ten to fifteen minutes at a time, making the possibility of involving PA in a daily life much easier.

Another misunderstanding regarding the need of physical activity in the daily life of each individual is the idea of what moderately and vigorously intense PA really mean. According to the National Heart, Lung, and Blood Institute (NHLBI) (2009) a vigorously intense activity is definable in an activity that substantially increases the heart rate of the individuals and the level of sweating, and when a person can't say more than a few words without stopping for a breath during PA. A moderately-intense physical activity refers to any activity that allows an individual to work hard enough to raise their heart rate and break a sweat, but at the same time being still able to carry on a conversation (Haskell, Lee, Pate, Powell, Blair, Franklin, Macera et al., 2007). A physically active lifestyle has been demonstrated to have similar health promotion and weight loss benefits that a structured and planned exercise program (Pratt, 1999; Braunstein, 2004). This lifestyle advantage to be easier to perform, not requiring expensive equipment, and being easily integrated in the normal daily life of an individual (Fahey, Insel, \& Roth, 2003). The positive effects of physical activity are present also in obese populations because an active lifestyle can ameliorate the hazards of obesity (Lee, Sui, Blair, 2008). Many authors in the last two decades have shown how a consistent active life can have a positive effect on the 
psychological well being of individuals and how being inactive instead is detrimental to it (Leith, 1994; LaPerriere, Ironson, Antoni, Schneiderman, Klimas, \& Fletcher, 1994; Miles, 2007).

Physical activity and fitness have been associated with academic achievement with a correlation between vigorous activity and higher grades, moderated by fitness in females but not in males (Kwak, Kremers, Bergman, Ruiz, Rizzo, \& Sjöström, 2009). Kristjánsson, Sigfúsdóttir, Allegrante, and Helgason (2009) found, studying their sample of 6346 students (respectively $51.4 \%$ girls $48.6 \%$ boys) all between 14 and 15 years old, that the health behavior indicators they analyzed were positively and strongly related to greater levels of school contentment. This effect has been found as possibly caused by healthy lifestyle, at least in some part, and strongly related with academic achievement $(\beta=.21, \mathrm{t}>1.96)$. These authors also found that the three indicators analyzed in their study were also associated with better academic achievement. This study also showed how BMI had little negative and direct relationship with school contentment $(ß=-.03, \mathrm{t}$ $>$ 1.96) and a similar, but little stronger, relationship with academic achievement for this indicator $(B=-.05, \mathrm{t}>1.96)$, with only a little part of the latter relationship due to mediation through contentment with school. Although both of these relationships are statistically significant $(\mathrm{P}=.01)$, neither one of those can be defined as strong. The second indicator, sedentary lifestyle, has been found directly and negatively related with both contentment with school $(\beta=-.05, \mathrm{t}>$ 1.96) and academic achievement $(B=-.11, \mathrm{t}>1.96)$, with the latter relationship due to mediation through school contentment only for a $10 \%$. The last indicator, physical activity, instead has been found positively related with both school contentment $(B=.11, t>1.96)$ and with academic achievement $(B=.08, t>1.96)$, with $20 \%$ of this last relationship accounted for by the mediation with school contentment. 
The relationship between PA and academic achievement has been shown by Stevens, Stevenson, and Lochbaum (2008) in their work with young children, showing the significant and positive relationship between PA and both mathematics and reading achievement. Similar results have also been found by Fox, Barr-Anderson, Neumark-Sztainer, and Wall (2010) looking at middle and high school students and at their participation in either physical activity or sports or both. Besides the many positive behaviors associated with PA, a correlation was found in relation to the increase of unhealthy behaviors, such as smoking habits, marijuana use, and television viewing (Pate, Heath, Dowda, \& Trost, 1996). Pate et al., (1996) also found an association between a low level of PA and the development of inappropriate dietary behaviors. High levels of PA was interestingly associated with some increase in alcohol consumption but only among female students, while instead it was unrelated to the drinking habits of male students. These authors also suggested analyzing these results with caution because in their study, due to the methodological structure of it, it was not possible to determine whether the relationship found between PA and the other health behaviors was a cause-effect relationship or not (Pate et al.).

\section{Nutrition and dietary behaviors}

Along with an active lifestyle, nutritionally balanced diets are the basics for a healthy life and a decrease of related diseases (USDHHS, 2000; 2005; 2008), whereas poor diet and physical inactivity are becoming main characteristics of the U.S. population (Driskell, Kim, \& Goebel, 2005). The World Health Organization (WHO) defined these two as interactive behaviors (WHO, 2002).

Although the Dietary Guidelines for Americans (USDHHS, 2005) provide guidelines for a healthy diet and nutritional behaviors, most U.S. adults do not follow such guidelines (Anding, 
Suminski, \& Boss, 2001). Jabs and Devine (2006) have sustained that time scarcity and the feeling of not having enough time have helped change in the way people consume food and develop nutritional patterns associated with a less healthy diet. According to these authors, these patterns are associated with less healthy diets are potentially becoming part of the origins of obesity and related health problems. One of the reasons for this phenomenon is, according to Brownell and Battle Horgen (2004), due to the economy, which is making more inexpensive and more easily findable "unhealthy food" characterized by its being tasty, cheap, fast, appealing and packed, while making the "healthy" food much harder to find and more expensive. Additionally, healthier food is less convenient because it is generally fresh and ready only after some cooking time. Popkin (as cited by Brownell \& Battle Horgen, 2004) has defined five nutrition patterns characterizing different countries in different times. Among them, the one which seems to better represent the situation in the U.S. is the so called "Nutrition-related Non-communicable Disease," characterized by diet high in fat and sugar and low in fiber, with a consequential increase of the obesity levels.

According to Kant and Graubard (2006), in the last three decades it has been found that an increase in energy intake has not been followed by an increase in the energy expenditure, with the consequence of a lack of balance, which can also be one of the reasons for the increased presence of obesity in the U.S. population. These authors also noticed that many behaviors related to food consumption have played a role in the energy intake. Some of those behaviors are the size of portions, skipping breakfast, snacking, the frequency of meals, the habit of eating outside, the decrease of homemade prepared food, meal time with family, and the increase of the utilization of convenience or ready-prepared foods. Other authors noticed sweetened-soft drinks were responsible for this increase across ages (Nielsen, Barry, \& Popkin, 2004; Kvaavik, 
Andersen, \& Klepp, 2005). It was also found that non-homemade foods, included college provided meal plans, generally contain more energy than needed and are less nutritional than the one prepared at home (Guthries, Lin, and Frazao, 2002). Although many researches confirmed these findings and added facts such as that late intake of food is detrimental either for the control of the total diary intake (de Castro, 2004) or for the control of weight (Eng et al., 2009). The dietary habit of having late meals and snacks is still part of the U.S. adult population nutritional rhythm, and overall for the college population.

The dietary guidelines for Americans (USDHHS, 2005) clearly suggest consuming at least 5 servings of fruit and vegetables per day. Although this suggestion is widely commonly known, analyzing data derived from the 18,359 respondents of 2003 Behavioral Risk Factor Surveillance System (BRFSS) for adults aged 18 to 24 years, McCracken et al. (2007) found that $78.4 \%$ of their sample consumed fewer than the suggested amount of fruits and vegetables per day. Although the majority of respondents within this national representative sample were classified as normal weight $(60.3 \%)$, the prevalence of being overweight and obese were respectively $26.1 \%$ and $13.6 \%$. Along with the decrease of fruit and vegetable intake, the U.S. population is also being represented by an increase in fast food consumption. Studies have confirmed the generalized idea that fast food consumption lead to weight gain and poor dietary intake (Bauer, Larson, Nelson, Story, \& Neumark-Sztainer, 2009).

With the goal of examining determinants of dietary intake and weight status, Larson, Neumark-Sztainer, Story, Wall, Harnack, and Eisenberg (2008) analyzed data coming from the project EAT - "Eating Among Teens” (Neumark-Sztainer, Wall, Guo, Story, Haines, \& Eisenberg, 2006a; 2006b). In their sample constituted by 1,686 high school students, respectively 935 females and 751 males, surveyed along a period of five years $(1998-1999 ;$ mean age $=15.9$ 
years and 2003-2004; mean age $=20.5$ years). These authors found that fast food consumption has also been seen increasing in the transition from adolescence to young adulthood. Specifically, they found that the proportion of males reporting frequent fast food intake went from $23.6 \%$ to $33.0 \%$ (change $=9.4 \%, \mathrm{t}=4.56, \mathrm{p}<.001$ ), whereas the proportion of females did not significantly increase (change $=2.3 \%, \mathrm{t}=1.41, \mathrm{p}=.16$ ), going from $20.5 \%$ to $22.8 \%$. Driskell, Kim, and Goebel, (2005) found in their sample that $95.1 \%$ of lower-class and $91.9 \%$ of upper-class students were typically eating at fast-food restaurants six to eight times weekly. Sneed and Holdt (1991) found that the most influencing factors that lead student eating patterns in their study were convenience,53.4\%; taste, 42.9\%; cost, 40.3\%; health, 31.9\%; weight control (gain, loss, maintenance), 23.5\%; and family/friends, 5.7\%. These authors also found a relationship between factors such as menu choices, cost, and convenience and the number of meals eaten in fast-food restaurants.

Along with the findings of Sneed and Holdt (1991), Brownell and Battle Horgen (2004) explained this constant increase in fast food consumption with the idea that fast food is perceived by the general population as answering to many of their needs, such as taste, work constriction, need of more leisure time, and consequentially less available cooking time. Fast food chains also answer many of the society needs creating jobs and helping the local economy.

Along with this nutritional path, the relevance that snacks are starting to have in the U.S. population eating habits is evident in the constant increase of the percentage of the population reporting eating three or more snacks per day (Cleveland, Goldman, \& Moshfegh, 2005).

Although the possible positive effect that snacking can have on the daily nutrition of individuals, researcher have also noticed that they can increase the total amount of food/calories intake, and potentially influence the development of obesity (Marmonier, Chapelot, Fantino, \& 
Louis-Sylvestre, 2002); therefore it is necessary that, instead of limiting the snacking itself, it is important to improve the quality of those snacks in terms of nutritional factors (Sebastian, Cleveland, \& Goldman, 2008; Cleveland et al., 2005). This necessity is also de to the fact that, as sustained by Brownell and Battle Horgen (2004), the food eaten between meals represents a continuing growing part of the total caloric intake of the general population.

Along with these nutritional behaviors, de Castro (2004) also noticed that the intake of food by individuals vary along the day, showing an increase in the amount of food ingested around the evening. Starting from a definition of high and low density food based on the amount of calories $(\mathrm{kj})$ per total weight $(\mathrm{g})$ of the specific food. Examples of high density foods are fat and proteins. de Castro (2004), in her study, showed that early morning intake of less dense food is particularly satiating and facilitates the limitation of the general intake, whereas late evening intake seems to not be particularly satiating, with the consequence of an increase in quantity, and indirectly of the total daily intake. De Castro (2004) stated that based on these findings, improving larger low-density intake in the morning, and reducing high-density intake in the evening could help to fight obesity.

\section{Use/misuse/abuse of alcohol and its products}

The consumption of alcohol is a characteristic behavior that predominating the lifestyle of the western society's population (Schröder, Morales-Molina, Bermejo, Barral, Mándoli Soler, Grau, et al., 2007). The consumer of alcohol is not necessarily clinical or negative, overall because a moderate use of it is also included in the Dietary Guidelines for Americans (2005), which states that the use of alcohol can have a beneficial or harmful effect depending on the amount consumed. Many epidemiological studies have anticipated the predominance of alcohol use and related disorders among the U.S. adult population (Schulte, Ramo, \& Brown, 2009), 
even more possible with an early-life use of alcohol and other drugs. The Substance Abuse and Mental Health Services Administration (SAMHSA)'s National Survey on Drug Use and Health (NSDUH; SAMHSA, 2008) reported a very high use of alcohol among the U.S. adult population. The 2008 National Survey on Drug Use and Health showed how $70 \%$ of the 18 year olds or older in the U.S. population had used alcoholic beverages in the previous year, with a little increase from the 2007 survey (69.7\%). Men seem to drink more than women (respectively $74.8 \%$ and $65.5 \%$ in 2008). This survey defines as "current drinkers" (i.e., reported drinking at least one alcoholic beverage in the past month) $55.7 \%$ of the US adult population with a peak in the $18-25$ years old population, which founds $62.2 \%$ of this population being "current drinkers". Along with the definitions of binge drinking as five or more drinks (or four for women) per sitting and of heavy drinking as five or more drinks (or four for women) at least five times in a month, the 2008 National Survey on Drug Use and Health showed how $24.9 \%$ of the 18 years old or older U.S. adult population experienced binge drinking in the previous month and how 7.5 of the same population experienced heavy drinking.

Based on this data, according to Shulte et al. (2009) among the adult population, drinking patterns differ by age and gender, but the total presence of alcohol use/misuse is heavily present in both genders and it is seen as increasing within the total general population. These authors also report, as the National Comorbidity Survey (NCS) estimates, that about $12.5 \%$ of men and $6.4 \%$ of women during their lives will meet diagnostic criteria for alcohol abuse. The high presence of alcohol within the college population, linked to the idea that "college behavior" can protract during the adulthood, make relevant the necessity to address this behavior with interventions targeted to a more moderate consumption as recommended by the Dietary Guidelines for Americans (2005). 
Schröder et al. (2007) in their sample based on 3054 Spanish people, respectively 1491 men and 1563 women between the 25 and the 74 years old found that the consumption of alcohol was directly associated with the length of the waist circumference and with a higher risk of abdominal obesity in male portion of their sample. In their sample these authors found that $19.3 \%$ of men and $2.3 \%$ of women consumed alcohol with more than 3 drinks per day. This consumption of alcohol was found directly associated with total energy intake in men $(\mathrm{P}<0.001)$ and women $(\mathrm{P}=0.001)$. These authors found that alcohol consumption significantly increased the risk of exceeding recommended energy intake in males. Along with these findings in their multiple logistic regression analysis they also confirmed that alcohol, and specifically more than 3 drinks of alcohol (>30 $\mathrm{g}$ ethanol), in addition to other factors such as, smoking, educational level, leisure- time physical activity, energy, and diet quality, increased the risk of abdominal obesity. Alcohol use on the young population has also been related to the development of behaviors that could be potentially detrimental to a positive academic experience, such as skipping school and acting inappropriate behaviors in class, affecting grades, and many others (Richardson \& Barrow, 2000; Greenblatt, 2000; Singleton \& Wolfson, 2009).

\section{Emerging Adulthood: Developmental Considerations}

According to Anderson, Shapiro, and Lundgren (2003), based on the idea that obesity and weight maintenance in general have become important public health issues, it is necessary to strategize ways to intervene, identifying key periods of weight gain during the life time. Based on this idea, in addition to the theory sustaining the persistency of some of the "college behaviors" in adulthood (Kelder, Perry, Klepp, \& Lytle, 1994; Sparling \& Snow, 2002), it has been considered the "college time" as a key period of the development and growth of these young individuals. Some authors described transitional moments, such as the passage from high 
school to college, times of personal social and environmental changes (Cullen, Kohely, Anderson, Baranowski, Prokhorov, Basen-Engquist, et al., 1999; Racette et al., 2005). According to Arnett (2000), the U.S. young population between the ages of 18 and 25 years old does not consider itself either adolescents or adults. This particular stage of the developmental process of each individual has been labeled by Erikson (1950) as "young adulthood". This period of life goes from 20 to 40 years old, whereas adolescence characterizes people between 13 and 19 years old. In order to better identify the specific step in life experienced by young population between 18 and 25 years old, Arnett (2000) defined this stage of the young population as "emerging adulthood", a middle stage between adolescence and the full adulthood, which Arnett considers to be a period of profound change and importance in the life of an individual. During this period young individuals experience many different possibilities in love, work, relationships, and worldviews. These new aspects of this developmental stage find their origins in the fact that during this period of life these individuals are relatively independent, having left the childhood and adolescence dependence behind themselves, and they are also not yet involved in the adult responsibilities and determination which leave the future undetermined and open to new experiences (Arnett, 2000). Unfolding this new theoretical framework, Arnett describes how this developmental stage is characterized by a difficulty in predicting the demographic status of this population. Many of the individuals going through this period of their lives experience one or more changes in their personal demographics, such as their residential status, their relationship status, and their career status. These changes are expressed by their moving out of parental home to go to college, by the fact that almost two thirds of the individuals in this stage experience a period of cohabitation with a love partner, and by experimenting school and work world, often concurrently. This instability and diversity in demographic status is 
already a characterizing aspect of this developmental stage. In their self-representation, these individuals do not perceive themselves as either adolescents or adults, living some ambiguity in defining their own roles toward themselves and society. During this period, there is the tendency to experience some identity explorations that becomes the third characterizing aspect of this developmental stage. Individuals are characterized by the tendency to look for an established role in the three main aspects of their lives: love, work, and worldviews. Love starts to get a serious role in the individual's life, characterized by exploration, experience, and commitment; work starts to be a fundamental part of both as source of economical sustain and as preparation for future. During this life stage, individuals are also characterized by the engagement in risk behaviors, such as alcohol misuse and smoking (Kelder, Perry, Klepp, \& Leslie, 1994; Ravert, 2009).

Another characteristic of this period is the fact that during this developmental stage, the individual experience changes in support systems and shifts in their interpersonal influences. With growing independence, family and social network and support start to assume different roles and their value start to weight differently in the individuals' lives (Nelson et al., 2008).

Although this life stage has been addressed and targeted using different nomenclature by many authors (e.g. Medalie, 1981), all of them considered this stage of growth as fundamental in the individuals' development. During emerging adulthood, individuals work toward the development of their self-identity while also exploring new ideologies and behaviors, which will help them to express this self-identity and individuality (Nelson et al., 2008). Authors have shown that the self-identity developed during the emerging adulthood stage, which also include healthy lifestyle values and behaviors, can be seen as a major precursor of a life lasting behavioral change (Storer, Cychosz, and Anderson, 1997; Miller, Ogletree, \& Welshimer, 2002). 
Gordon-Larsen et al. (2004) noticed that this period characterizes an enormous shift in the adolescents' lives during their transition to young adulthood, or emerging adulthood. Hogan (1978) described how the patterns developed in this period have more influence than the family background on the way young adults order key events during their life and during the life cycles they will go through. Understanding these motivations and ideologies characterizing individuals involved in the emerging adulthood developmental stage could be imperative to develop and enforce health-promoting intervention, which could be promoting specifically weight-related health behavior.

In defining the characteristics of the emerging adulthood, Arnett (2004) identified five characteristics typical of this developmental stage: a) identity exploration (IE), characterized by trying out possibilities overall in the realm of love and work; b) Instability (I), potentially due to IE; c) self-focused (SS); d) feeling in-between (FiB), transitional moment between adolescence and adulthood; e) age of possibilities (AoP), where hopes and dreams flourish, when people face occasions to change their lives. According to Larson (2008) these characteristics could also have some implications for nutritional behaviors during this developmental stage. Based on findings that show how health related behaviors developed in college would likely also influence the post college behaviors of individuals (Sparling \& Snow, 2002). This hypothesis can also be widened up to the other health behaviors that will be considered in this study.

\section{Identity Exploration.}

In emerging adulthood the individuals deeply explore personal values and their desires from life in the love and work realm (Arnett, 2004). In both of these realms there is a passage from short-terms to long-term relationships, with loves and job lasting longer and becoming more relevant in the life experience of the individuals. During this period, individuals set their 
own set of values and beliefs trying many different possibilities in these realms (Arnett, 2004).

While facing this process, also nutritional behaviors and habits needs to be modified based on the new and current life circumstances, eventually revaluating attitudes and behavior related to health nutrition based on the new-faced experience (Larson, 2008). Other behaviors could be part of the same re-evaluating process, with a change in the individuals' attitudes and in their habits related to a physically active lifestyle and alcohol-related behaviors. The use of alcohol could be affected by the developmentally common increase of the exposure to experimental behaviors seen as useful for growing and detrimental at the same time by different authors (Arnett, 2000; Dworkin, 2005; Park et al., 2006; Ravert, 2009; Schulenberg \& Zarrett, 2006). The attitude toward to physical activity could instead be influenced by the new identity and beliefs, which could also modify the attention paid to health and health behaviors.

\section{Instability.}

The exploration process of the individuals during this developmental life stage can also have as main result a constant modification of the life plan in terms of values, attitudes, locations, habits, and etcetera (Arnett, 2004). Individuals currently stay longer in their parents' house, also before getting married, and before being totally independent and out from the household. This prolonged period with parents can, during this stage, also influence the nutritional habits, shape attitudes toward physical activity as meaningful and purposeful part of life, and profile and incline these young individuals' view of alcohol use.

Every time these individuals modify the environmental situation by moving and changing homes, the modification of the cost and typology of food, location of restaurants, supermarket, and gym characterize the new situation as well. The landscape and the environmental opportunities to be active change and at the same time the alternatives to alcohol use. All of these 
modifications can also modify and influence the future and the present of all these health behaviors (Larsen, 2008), and eventually help these people to survive the transition to work.

\section{Self-focused.}

Emerging adults are more self-centered than adults or adolescents (Arnett, 2004).

Whereas during younger ages, these individuals needed to follow parental rule and those established by teachers, educators, bosses, and others, during this stage emerging adults will start to be able to develop and build their own life style, based on specific habits and rules. Among those habits, nutrition, physically active lifestyle, and alcohol use are considered among the most relevant (Anding et al., 2001; Nelson et al., 2009).

\section{Feeling in-between.}

This is a typical characteristic of this transitional developmental stage because individuals facing this moment of their life feel they have left adolescence, but have not completely reached adulthood (Arnett, 2001; 2004; Nelson et al., 2005). They are characterized by all series of uncertainties due to the difficulties that define their roles in society and in their lives and in their own families/group of belonging. Larson (2008) reported that having the perception of having reached some goals, such as self-responsibility, independent decision making, and financial independence, sign of an adult life stage (Arnett, 1997; Nelson at el.), can reduce the tendency toward risk-taking behaviors while experiencing the emerging adulthood life time (Nelson et al.). This sense of self-responsibility, according to Larson (2008) can also positively influence the health behaviors embraced by individuals in terms of nutrition. This influence can be easily enlarged to other health behaviors such as alcohol consumption and physical activity.

\section{Possibilities, hopes and dreams for the future.}

The fifth main characteristic of this developmental stage (Arnett, 2004) bring these 
individuals to perceive their ability and possibility to reach, one day, their goals and dreams, looking for a better life than their parents' (Larson, 2008). In terms of lifestyle, this period is a moment of improvement of the life style they have been previously experienced. This new life style can be developed also building behaviors, health related or not, that can be brought and experienced along all life, in all the three targeted realms, nutrition, physical activity, and alcohol consumption.

In agreement with Arnett's ideas (2000; 2001; 2004), Gordon-Larsen et al. (2004) considered the transitional period between adolescence and young adulthood as a period of major life event changes, mainly related to the move from the parental home to the complete residential independence. Medalie (1981) affirmed that people between 17 and 22 years old are normally involved in a transitional period of their lives. This transition is particularly evident in the "Western life cycle" (p.75), where this young men and women need to leave their childhood and experience and create a "new life structure suitable for adulthood" (p.75). According to Medalie (1981) the "undergraduate years provide a moratorium on final identity commitments and a bridge to adulthood" (p.75). Arnett (2000) sustains that a central task of this period of those young men and women is the exploration of their identity. It s also manifested, according to some authors, as a high level of experimentation, often merging in reckless or deeply risky behaviors, such as smoking and substance use (Arnett, 2000; Park, Mulye, Adams, Brindis, \& Irwin, 2006; Ravert, 2009).

Arnett $(2000 ; 2004)$ justifies this behavioral experimentation with two possible explanations not necessarily mutually exclusive. The two possible explanations offered by Arnett (2000; 2004) see experimentation as either part of the path through the developmental tasks faced by this young population to discover their own identity, or as a way to not lose the 
opportunity to do something that in adulthood won't be seen as an acceptable and possible option. Park et al., (2006) also see this period of the life span characterized by two more factors, one the transitions to the adult role and the other the detachment from the safety net that supported them when adolescents and younger children. Ravert (2009) found that most of the college population participating in his study considered an important aspect of their current life stage the "exploration in terms of pursuing new and novel physical, social, and intrapersonal experiences" (p.386).

Despite the fact that during this age, the young population's health is represented by much worse health indicators and that most of the risk behaviors have potential repercussions on the future healthy condition of this population, according to Park et al., (2006) emerging adulthood healthy conditions have received far less attention than adolescents' health conditions. Between adolescence and young/emerging adulthood, mortality rates doubled, with peaks in the early 20s, and being obese and overweight are seen as the second leading causes of death. Supporting this data, there is an observation of Lewis et al., (2000) who found that the biggest weight gain in their population happened in the early 20 s, compared to the one happening in the thirties.

Researches based on national surveys have identified how one of the periods with the highest risk of excess weight gain is the transition period between adolescence and adulthood. Although one of the reasons, as showed by Gordon-Larsen et al. (2004), is represented by the fact that within this population, the majority of people does not meet the recommendations in terms of physical activity, only little research among young adults has been done regarding diet and physical activity patterns (Nelson et al., 2008). Along with other factors, obesity and substance abuse, also with higher rates of injuries, homicides, and mental health, are considered 
among the most relevant leading path towards to lack of health among this cohort of people (Park et al., 2006). These factors are also sustained by the tendency of this aged group people to experiment at-risk behaviors.

During this period of experimentation (Arnett, 2000 Park et al., 2006; Ravert, 2009), the level of alcohol use is high both in college and non-college population. This phenomenon has related consequential associated risk behaviors, such as driving a motor vehicle after drinking, that pose risk to themselves and others. Hingson et al., (2005) found that among 18 and 24 year old young adults, the number of alcohol-related death in U.S. between 1998 and 2001 overcomes the age group appropriate population increase and that the unintentional alcohol related injuries deaths increased by $9 \%$. All these factors make this period of life an intense and extremely relevant period of the individual development.

\section{Transition to College: the Emerging Adult Becomes a College Freshman}

The first semester of the freshman year of college is considered a critical period in young adults' lives because they are required to make personal choices and initiate behaviors that can impact their health and well-being (Newton et al., 2006). According to Crissey (2008), based on the data recollected by the U.S. Census Bureau, $27 \%$ of the adults 25 years old or older have a bachelor's degree or more education and the $10 \%$ reported an advanced degree. With these high percentages of emerging and young adults enrolled and who have been enrolled in college, as sustained by Zimmerman (2002), it is possible to consider higher education institutions as potential bridges to reach emerging adults in order to develop, increase, and improve their health behavioral habits. Among the emerging adult population between 18 and 24 years old, a large proportion is enrolled in postsecondary institutions, being part of the college student population. According to the Department of Education, in 2008, 18.4 million students enrolled in 
"postsecondary degree-granting institutions", over 3 million students more than in 2000 (Snyder, Dillow, \& Hoffman, 2009). According to Mellen (2007) the number of high school students that directly enrolled in college right after their graduation continues to increase.

Medalie (1981) defined the change between high school and college as the transition from a structured environment to an environment characterized by a mix of people and by a diverse type of expectations; transition also characterized by the process of leaving childhood and starting to be responsible for their decision making and for caring about their own physical and psychological needs. In her still contemporary developmental college structural growing path, Medalie defines the characteristic tasks of freshman year as "disinvestment and investment". She sees the freshman living the new experience of separation from the parents and from their house getting more and more involved into the college community. This new investment in the college community could be the origins of an increased importance of peers in the development of new behaviors with the goals of better fit-in and to be better accepted. Because most universities make their incoming freshmen students live in the residence halls where they will spend the majority of their freshmen year living, socializing, studying, sleeping, and eating (Kasparek, Corwin, Valois, Sargent, \& Morris, 2008), these structures assume a relevant part in the developmental path of these emerging adults, and consequentially in their process to fit in and being accepted.

Because of the high relevance of health and health behaviors development during this period of life, many authors (i.e. Ferrara et al., 2008; Nelson et al., 2008) considered the college experience and the campus setting itself potentially becoming relevant factors to consider in developing health behavior interventions. These health-promotion interventions could find a 
good setting within college campuses for health-promotion interventions, as a starting point for the development of current and also future health behaviors.

A study in Canada addressed these health behaviors on incoming freshman following their entire 108 male sample from the summer previous to the beginning of the freshman year to the entire academic year (Pullman, Masters, Zalot, Carde, Saraiva, Dam, et al., 2009). It was found that between the summer prior to and the end of the first year, the participants experienced a significant weight gain (about 13 pounds) with significant increases in BMI, body fat, and other anthropometrics. These changes seem to be mainly explained by a strong increase of physical inactivity and by an increase in alcohol consumption. Anderson et al. (2003) found a statistically significant weight gain in their freshmen participants that tended to occur at the beginning of the year right after the beginning of their college careers. Additionally the number of people that fit the definition of overweight and obese, toward to the end of the Spring semester, doubled.

To better understand how to support these students during this transition, with the final goal of building long lasting healthy life style, it might be useful to look at their freshmen year and at the health behaviors developed during this step of their life with the goal of addressing the weight gain experienced by these young people. It has been found that this population gain weight 6.7 times more than the general U.S population (Kaspareket al., 2008).

\section{First Year Students' Health Behaviors}

According to Racette et al. (2005), students, during the first year of college, see weight gain as a problem. Although this has been reported in many studies, many more show how more and more students eat less and less fruit and vegetables, engage in less and less physical activity, and drink more and more alcohol (i.e. Racette et al., 2005; Hingson et al., 2005; Keating et al., 
2005). Even though many studies have supported with findings the weight gain that is normally experienced by students during this transitional year (i.e. Cullen et al., 1999; Kasparek et al., 2008), a controversy about the "Freshman Fifteen" phenomenon is still alive, with who sustains that it is a real weight gain occurrence (Levitsky, Halbmaier, \& Mrdejenovic, 2004) and who instead consider it just a myth (Graham \& Jones, 2002).

Associated with weight gain, besides a poor nutrition as reported by many different authors, there were alcohol use and lack of physical activity (Andreson et al., 2003; Kasparek et al., 2008; Pullman et al., 2009; Butler, Black, Blue, \& Gretebeck, 2004). During this transitional period, physical activity, alcohol use, and nutritional habits change and, according to Cullen et al., (1999), they change differently among males and females. These authors also sustain that college freshmen gain 5.5 times more weight than the general population.

This legendary weight gain process is commonly named "Freshmen Fifteen", and it is perceived and experienced by many first year college students (Mihalopoulos, Auinger, \& Klein, 2008). This phenomenon, however, still lacks a strong body of scientific evidence about this amount of gained weight (Mihalopoulos et al.).

According to Hoffman, Policastro, Quick, and Lee (2006), potential origins of this phenomenon are the behaviors developed during the first college year characterized by the increase in energy intake and the decreased level of physical activity. These authors found in their sample, constituted by 67 freshmen ( 32 males and 35 females), that the weight gain was respectively $1.32 \mathrm{~kg}( \pm 5.04)$ and $1.28( \pm 2.74)$ for men and women, and only a third of this sample (33\%) was characterized by a weight gain of fifteen pounds or so.

Looking at freshmen and at the relationship between alcohol use, nutrition, and physical activity, Pullman et al. (2009) decided to monitor this population along the entire first year, with 
three study points: the summer prior to the beginning of the academic year, during the fall, and during the winter. Their sample was a convenient sample of 108 , from an original sample of 128 , students, who need to fit in the including criteria represented by being healthy male students between 17-20 years of age, accepted to the university, and with the intention to live away from home (Pullman et al.). Although these authors did not find statistically significant differences in terms of energy intake in their study, they found statistical differences in terms of food servings consumed, including the amount of alcoholic drinks consumed. Among the surveyed students, some differences have been found between the summer prior to and the end of the freshmen year, during which these individuals experienced a significant weight gain of $3.0 \mathrm{~kg}$ accompanied by an increase in some anthropometric measurements, such as BMI, body fat, and waist circumference.

The study did not show differences, statistically significant, in the amount of alcohol consumers during the freshman year. Instead, among those students who reported alcohol consumption $(\mathrm{n}=85)$, they found a statistically significant difference in the number of drinks had per sitting between summer and winter study visits, but not between the fall and winter (summer, $7.33 \pm 1.0 ;$ Fall, $10.2 \pm 1.18(\mathrm{p}<0.0001)$; and winter, $9.38 \pm 1.0 ;(\mathrm{p}<0.0001))$. Still statistically significant were the differences found in the frequencies of drinking and in binge drinking, as found previously by Pliner and Sanders (2008). The frequency of binge drinking, thought as the number of times per week (means $\pm \mathrm{SE}$ ), increased between the summer and winter, but did not significantly change between fall and winter (Summer, $1.00 \pm 0.09$; Fall, 1.38 $\pm 0.10(\mathrm{p}<0.0001)$; and Winter, $1.27 \pm 0.09(\mathrm{p}<0.0001))$. Although no significant differences were found in intake of energy or nutrients between any of the study visits, the results of the 
study seem to show a tendency of better nutritional habits held by these students previous to their college experience.

In addition to these findings, in their study, Pullman et al. also found out that the increases in the anthropometric measurements in their 108 males were accompanied by statistically significant $(\mathrm{p}<0.0001)$ changes in sedentary behaviors, with more computer and studying and less physical activity. According to several authors, many environmental and social influences guide students to behave in particular ways, overall in terms of food, drinking and exercise habits and choices (Lowry, Galuska, Fulton, Wechsler, Kann, \& Collins, 2000; Matvienko, Lewis, \& Schafer, 2001). These behaviors can also have long lasting consequences on weight and health (Hoffman, Policastro, Quick, \& Lee, 2006).

\section{Health Behaviors in College Students}

Sparling and Snow (2002), in their study about physical activity patterns in college alumni, graduated between 1988 and 1996, surveyed a total of 367 recent alumni, 30\% of who were women. About half of these respondents $(50.7 \%)$ had taken the health education course, $37.9 \%$ the fitness concepts course, and $4.6 \%$ had completed both courses whereas $6.8 \%$ neither course. Among their sample, these authors found no differences between alumni who took the fitness course and the health based course, as well as no differences were found in age, height, weight, and exercise patterns as a college senior, current PA patterns, or attitudes toward exercise. This data supports the idea that there is little long-term impact of classroom based health or fitness education courses, which are not tailored to individual needs.

The study by Sparling and Snow (2002) originated two relevant findings: the fact that $84.7 \%$ of the regular exercisers as college seniors were as active or more active at the time of the survey, and that $81.3 \%$ of the non-exercisers were about the same or even less active at the time 
of the survey. Consequentially to these two findings, these authors also found that the individuals reporting to be regularly active in college, were likely to participate in recommended levels of PA, regardless the level of it, in the years after graduation. They also found that those who reported to be "more active now" or "about the same" had reported also significantly less weight gain than those who reported to be "less active now" $(\mathrm{p}<.001)$.

According to Healthy Campus 2010, whose main goals were to increase quality and years of healthy life and eliminate health disparities within the campus life, the six priority areas that need to be the goal of the improvement in health related college issues are: injuries and violence; tobacco use; alcohol and other drugs; sexual behaviors; unhealthy diet; physical inactivity. These behaviors are also interrelated among each other (Eaton, Kann, Okoro, \& Collins, 2007). Racette et al., (2005) analyzed 764 college students (53\% women, $47 \%$ men) including 274 freshmen in 1999 and 490 freshmen in 2000 , with a mean $( \pm$ SD) age of $18.1 \pm 0.3$ years. In their sample these authors found an inverse correlation between fruit and vegetables intake and fast-food consumption $(r=-0.43, p<.001)$ and with fried food $(r=-0.12, p=.009)$. At the end of sophomore year, only 290 of the original sample returned for follow-up assessments, and within this sample, the authors found higher weight and BMI. The body weight increased in $70 \%$ of the sample, whereas decreased in $26 \%$ of the students and stayed the same in $3 \%$. For those students who gained weight, the mean increase was $4.1 \pm 3.6 \mathrm{~kg}(p<.001)$.

With this statistically significant increase in body weight and Body Max Index (BMI), Racette et al., (2005) showed also the necessity of intervention. More than $55 \%$ of participants, for example, were in the precontemplation stage in terms of adopting healthier eating behaviors, according to the dietary guidelines (USDHHS, 2005) at both the beginning of freshman year and at the end of sophomore year. 
Nelson et al. (2008) in their meta-analytic work, among many studies, found some decreases in moderate to vigorous physical activity in girls (from 5.1 to $3.5 \mathrm{~h} /$ week aged from 16 to 20 years), and increases in leisure time computer use in boys (from 10.4 to $14.2 \mathrm{~h} /$ week aged from 16 to 20 years). In boys, the decline of PA only started after high school graduation. These changes in PA started during the transition between the last two years of high school and the first years in post graduation.

Keating, Guan, Castro Piñero, \& Bridges (2005) showed that among the college population, only $50 \%$ of it was engaged in physical activity. This data shows a big level of inactivity among this young population overall since the fact that no more than $36 \%$ of these students were meeting the adequate amount of PA suggested by the ACSM and CDC guidelines. This data definitely shows a strong need for interventions able to help increase the engagement and involvement of the college population in PA. Another two relevant findings in this study were that college students were involved mainly in activities in which they already feel competent and that walking, as form of physical activity, was not too common between these emerging adults. Keating et al. also found that for this population, in order to maintain and increase physically active behavior, it was fundamental the presence of social support. The type of this support depended on the gender, with peers having a major influence on the male population and family playing a bigger influence on the female counterpart.

In terms of meeting the dietary recommended guidelines, Anding et al. (2001) found that only $43 \%$ of college women included in their study followed at least one of these guidelines, whereas $32 \%$ and $10 \%$ of this population followed respectively two or three of these. Racette et al. (2005) found that only one third of their college students sample was consuming the recommended serving of fruits and vegetables. 
Driskell, Kim, and Goebel (2005) analyzed a sample constituted by 261 students to find potential differences between lower level and upper level students in terms of "typical eating" and "physical activity habits". This sample was characterized by being constituted by 114 lowerlevel and 147 upper-level students, with a gender distribution between groups significantly different $(\mathrm{P}<.05)$ where men represented $21.1 \%$ of the lower-level and $40.8 \%$ of the upper-level students. These authors found some differences between lower-level and upper-level class college students related to their nutritional and physical activity habits showing the likelihood of few changes in these habits throughout the college experience. The most significant difference was represented by a higher $(\mathrm{P}<.05)$ percentage of upper- level than lower-level $(59.9 \%$ vs 47.4\%) students typically snacking in the afternoon. Another statistically significant difference $(\mathrm{P}<.01)$ was found between groups in terms of number of meals had in university cafeterias, with respectively $74.8 \%$ lower-level and $84.4 \%$ upper-level. In terms of physical activity the most significant differences $(\mathrm{P}<.05)$ were observed among the participants in terms on the length of time they typically walked on the frequency of performing aerobic activities. Among the many reasons listed by these students as main reason for their engagement in physical activity, two were the most mentioned, "health" and "weight loss". Along with these two, "having fun", was the third of the three factors found to be characterized by statistically significant $(\mathrm{P}<.05)$ differences between the two samples. Specifically, around $60 \%$ of the lower class students listed "health" as the main reason, versus the $40 \%$ of the upper level class, who instead mentioned mostly "losing weight" ( $\approx 45 \%$ vs $\approx 25 \%$ of lower level). Lower level students $(\approx 20 \%)$ seem to prefer, compared to upper class $(\approx 10 \%)$, "fun" as main reason for their physical activity. 


\section{A Short Analysis of Different Types of Intervention Develop to Address the Problem}

Along with this recognized need of intervening on this public health issue, both for individual health and for the society as a whole, it has been sustained that the need of targeting the increase of physical activity, the improvement of diet quality, and the decrease of alcohol as main tools against this problem and toward a healthy lifestyle (Butler et al., 2004).

According to Blue and Black (2005) physical activity and healthy dietary habits are relevant targets for controlling chronic diseases. According to them, being able to modify these behaviors would realistically make it possible to manage and diminish body weight. Many interventions have been developed with the goal of addressing this issue and to improve these behaviors in different sections of the general population and using different tools. Many have been the interventions that have singularly addressed these behaviors, while much fewer are the number of the developed interventions that have addressed them from a multi behavioral perspective. In the last few years a growing body of this multi-behavior intervention is appearing in scientific literature.

\section{Physical Activity and Exercise}

Kahn, Ramsey, Brownson, Heath, Howze, Powell, et al. (2002), in their review of literature concerning the effectiveness of the interventions targeted to increase the level of PA, besides described the diverse typology of intervention that have been developed, also offered a compendium of those that have been proved to be the more effective. I $t$ also provides possible suggestion about the best option to embrace while developing a new intervention. Although few of the studies analyzed by Kahn et al. (2002) were addressing also other behavioral risk factors, besides physical inactivity, these authors based their suggestions and analysis only on the physical activity aspect. As a first distinction, these authors divided the intervention in three 
main families: informational approaches; behavioral and social approaches; and environmental and policy approaches. For each of these main groups they defined sub-families of interventions defining for each of them the effectiveness, the applicability, positive and negative effects, the economic aspect, and the barriers to the implementation of them.

"Informational approaches" are interventions designed to change behaviors of the individuals by offering information, using educational approaches, motivating people to change their behaviors and maintaining the new one over time, focusing mainly on cognitive factors, which precede the behavior itself. This educational approach is based on the provision of knowledge about the benefits of PA, about ways to overcome barriers and negative attitudes, and more. Among these types of interventions the authors listed the "point-of-decision", the community-wide education campaigns, mass media campaigns, and classroom based health education. The second main family proposed by Kahn et al., is defined "behavioral and social approaches", which are focused on teaching widely applicable behavioral management skills and by structuring the social environment in order to offer support to those individuals trying to change or maintain a certain behavior. Among these interventions, there are the so called schoolbased physical education (PE), the college-based health education and PE, the classroom-based health education focused on reducing TV and video games use, family-based social support interventions, social support interventions in community settings, and the individual-adapted health behavior change program. The third and last family is the "environmental and policy approach based", characterized by the provision of environmental support, opportunities, and cues to help individuals developing their behaviors based on the correlational findings that relate the increase of physical activity, and the availability/proximity of exercise setting/equipment. 
According to their findings, Kahn et al. identified few among these interventions that would help to develop new interventions targeted to reach the Healthy People 2010 Objectives for Physical Activity and Fitness (USDHHS, 2000). They strongly recommend a communitywide health education campaign, a school-based PE, and social support in community settings. These settings stress the role that can be played by multisite, multi-component interventions, and individual-adapted health behavior change intervention increasing physical activity. In their recommendations these authors also stressed the functionality that individual-adapted health behavior change and the creation and the enhancement of physical activity opportunity could have when combined with informational outreaching approaches.

Dishman and Buckworth (1996) based their meta-analytic review of studies on 127 studies and 445 effects they retrieved, showing a nearly normal distribution of effects among these studies. These authors found that the effect size was not different between men and women in their studies, age groups and white-not white individuals They didfind that race-based group tailored intervention were showing smaller effects than general population based studies. Intervention using mediated approaches showed larger effects (.91) than face-to-face interventions (.16); community-based (.82) and group-based interventions (.75) reported bigger effects than school-based interventions (.21) and individual-based (.16). Behavioral interventions were found having effect sizes larger (.92) than other interventions (from .10 to .21). Interventions characterized by mediated approaches and community or group delivery showed the larger effects. Differences were found between unsupervised PA and leisure-based activities (.78) compared to supervised physical activity and exercise programs (.23). Low to moderate PA based programs also show larger effects that vigorous PA based programs. No differences were found between programs of different lengths. Differences have been described using moderating 
variables not weighted by sample size, such as between intervention addressing one or more than one race; not in terms of delivery system, of the intensity of PA, or of the social delivery setting. No difference between supervised and unsupervised PA or between programmed and leisure PA were found. Less encouraging results came from the longevity of the effects of these interventions implying that all of them constantly needed to be implemented in order to maintain the effects of them. Among these interventions those characterized by behavioral modification approaches showed bigger effect size overall when combined with reinforcement and stimulus control (Dishman \& Buckworth, 1996).

Marcus et al., (2006) in their review looked at 149 different studies written between 1995 and 2005 that focused on interventions targeting the increase of physical activity behaviors in different populations, in different settings, and using different approaches. In terms of age groups, these authors showed evidences of how short-term PA increase among older adults, when the intervention goes beyond educational approaches. Instead, they found that most of the interventions targeted for children and adolescents were school-based. These interventions have shown potentialities to increase physical activity. These intervention had smaller impact than those targeting community based and family based programs and characterized by multilevel approaches. Among the analyzed studies, those addressing specific populations, such as underrepresented population, have been able to overcome many of their characteristic methodological problems that show some positive potentially useful results in terms of an increase in PA. These authors also offered a short description of the "status quo" of the research in physical activity related to work-place based interventions, related to interventions delivered with the use of the so called "mediated" approaches, and to interventions based on environmental approaches. In terms of interventions structured in the work place, these authors state that they 
generally show good results overall using individual tailored theory-based and/or environmental prompts, with the limitation of a scarce external validity. The authors considered mediated interventions, those delivered through means other than face-to-face delivery system such as by print material, via internet, via text, and via phone. These interventions showed moderate efficacy in increasing physical activity behavior regarding short-term effect. Although printbased interventions have been studied more extensively, many are the evidence of potential positive effects also of the other types of intervention. It is possible that these interventions could also produce positive effects on the long-term maintenance. The environment-based interventions showed some weaknesses, with space for growth in terms of their potential results, and in terms of their methodological structure. Marcus et al. also described how many of their studies showed positive results in terms of adoption of physical activity stressing the need for more research addressing the maintenance of these behaviors.

Keating et al. (2005) defined higher education as one of the best environments to improve the physical activity behaviors of people. Starting from this point of view, they review studies focused on interventions based on physical activity behavioral change addressing college students. In their review, these authors sustained that a strong limitation of these studies was due to the many different ways to measure PA, which did not allow having a consistent analysis of such a common dependent variable among the studies. In their study these authors looked at which stage of readiness to change their sampled college population was, stating that knowing where this population placed itself, would have been relevant in terms of the development of the intervention addressing, with different strategies, different levels of readiness. They found that higher amounts of these students were included in the maintenance stage (20 to 30\%), but that according to other previous studies the majority of college students were not involved directly in 
PA. They might have been thinking about it, but were not involved yet, being found mostly between precontemplation (10 to $15 \%$ ), contemplation (25 to $30 \%$ ), and preparation (15 to $20 \%$ ). These authors found studies mostly based on curriculum models with sample sizes based on hundreds students, but with different time frames and not all of them characterized by follow up strategies or free opportunities to increase PA behavior. What has been found, was a moderate effect on the short term, with a not clear effect on the long term in two studies out of three, since the third was not concluded yet by the time of their study. Along with these findings, Marcus, Dubbert, Forsyth, McKenzie, Stone, Dunn, and Blair (2000) showed the importance of understanding how the maintenance of PA could be increased and the importance of finding a common way to measure PA in order to compare the findings of the numerous studies focusing on PA. These authors also addressed the necessity of targeting different ages in specific ways. They also showed how short-term and long-term behavioral change interventions face different barriers creating interventions targeting physical activity. In conclusion, they argued that the socalled “one-size-fits-all” programs are rarely as effective as the targeted interventions, which focus on a specific population approaching it in a specific way.

Previous it has been demonstrated that people who normally focus on the "here-and-now" is negatively associated with health behaviors. Focusing on short term consequences of their actions, these people are more likely engaged in risky and unhealthy behaviors. Instead people with more attention to their future in a more "long-term" perspective have been found to be more likely engaged in healthier and safer behaviors (Strathman, Gleicher, Boninger, \& Edwards, 1994; Keough, Zimbardo, \& Boyd, 1999; Zimbardo \& Boyd, 1999). Hall and Fong (2003) started from the premise that a lacking part of the previous studies, that instead would be fundamental in increasing the potential long-term effect of PA intervention, is the connection 
between current behaviors and future consequences within the realm of physical activity and exercise. With the goal of fighting the lack of long-term effects very commonly presented in PA interventions, they started to look at the determinants of this type of effects. These authors started from the idea that stronger would be the connection between current behavior and future consequences, higher would be the long-term effects of behavioral change interventions. In their two studies, they demonstrated how the efficacy of PA maintenance intervention could be improved adding to these interventions a time perspective component, represented by three, 30min long weekly classroom sessions during the 10-week fitness class. The content of these classes focused on helping participants, through education and activities, to become more conscious of the long-term consequences of their current actions. Concurrently they would also become more responsive to them, keeping these cognitions active during their participation in, or decision-making around PA (Hall and Fong, 2003).

In both studies, their samples respectively 18 and 81 participants, were predominantly female college students (17 and 77) with an average age of 21 years old $(M=21.3 ; \mathrm{SD}=1.9)$ and both showed a great retention rate $(100 \%$ and $97 \%)$. In their studies they evaluated three different conditions: one addressing the time perspective, one addressing goal setting, and the other without any time references. In the pilot study, these authors found a statistically significant group $X$ time interaction $[F(2,15)=5.73, p=0.014]$, with individuals in the time perspective group exercised $10.92 \mathrm{~h}$ longer than the other two groups. In the follow up study, these individuals showed to have developed an increase in different areas, such as their long term thinking about exercise and in their engagement in vigorous PA. Although there were innovative findings, this study has the limitation represented by the small sample size and by the predominance of females. 
The second study maintained the same structure with a bigger sample. Although no significant interaction group $\mathrm{X}$ time was found between pre and post intervention. A nearly significant effect was found at the six month follow up $[\mathrm{F}(2,53)=2.84, \mathrm{p}=0.067]$, with the individuals who had received the time perspective intervention exercising longer than the other two groups. A strong effect was found in terms of engagement in high intensity PA $[F(2,66)=5.70, p=0.005]$ with the time perspective participants exercising harder than the others. Similar type of effect was found for strength and flexibility engagement $[\mathrm{F}(2,51)=4.044$, $\mathrm{p}=0.023$ ] in favor of time perspective individuals. This study provided an important applied innovation showing how PA interventions could be improved and made more effective adding this perspective with the goal of increasing the promotion of PA and also its maintenance.

\section{Nutrition}

Simons-Morton, Obarzanek, and Cutler (2006) in their work sustained the relevance with high-risk patients in clinical practice of medication, diet, and life style interventions. They also sustained the necessities of more research to study the effectiveness of diet and lifestyle interventions delivered in practice and, at the same time, the need of verifying the long-term effectiveness of medication-based interventions. Multiple strategies have been used trying to reach effective weight loss, with the specific goal of limiting the energy intake. Wu, Gao, Chen, and van Dam (2009) sustained the existence of different types of diets, and, although some study would seem to lead toward low-carbohydrate dietary pattern as the most effective, there is a lack of evidence to determine which is the most effective dietary pattern to follow in order to lose weight.

Many authors are convinced that the college student population is the most subject to weight gain and body fat (e.g. Anderson et al., 2003; Butler et al., 2004; Graham \& Jones, 2002; 
Hoffman et al., 2006; etc). This phenomenon would be because of the many influencing factors that characterized the life of these individuals in the college, such as the cafeterias "all-you-caneat" style, the lack of knowledge about nutrition, the increase of alcohol, and the decrease in PA (Hoffman et al., 2006; Holm-Denoma, Joiner, Vohs, \& Heatherton, 2008; Hull, Morrow, Dinger, Han, \& Fields, 2007; Levitsky, Halbmaier, \& Mrdjenovid, 2004). Many authors sustained the idea that colleges need to intervene, develop, and implement programs focusing on teaching students how to manage their weight while addressing PA and nutrition (e.g. Jozkowski, 2007; Lowry et al., 2000; Nelson et al., 2008; 2009; Racette et al., 2008; etc). Along with this idea Jozkowski (2007) also sustain that college students are the population who can be influenced the most by interventions addressing physical activity and nutrition behaviors.

In their meta-analytic work, Dansinger, Tatsioni, Wong, Chung, and Balk (2007) revised forty-six studies that focused on dietary counseling. These studies were randomized trials that reported data on the effects of the advice to change dietary patterns aimed to change weight or BMI (Body Mass Index), compared with the effects of control interventions, characterized by usual care or minimal intervention. These authors did not consider all the studies where exercise was the only interventions among which weight loss was not the main goal of the study. Four of these 46 studies included: 63 treatment groups, data from 136 time points, 6386 individuals who were exposed to dietary counseling, and 5,467 individual who, instead, received usual care. Specifically, the studies in this group were differentiated in 26 treatment groups where diet was the only strategy and 37 where diet was accompanied by exercise. In 42 of these treatment groups, exercise was promoted in addition to diet. The goal of all these studies was weight loss by limiting fat and/or calories intake, specifically 17 of these were targeting to limit general calorie intake, whereas 25 were targeting to limit fat intake. Among these studies, 18 used group 
meetings, 13 used individual meetings, 11 used a combination of the two, and 3 used Internet. Two of these 46 studies did not specify the way in which the intervention was delivered. These authors found that weight loss based on dietary counseling produced modest net weight loss if compared to the weight loss obtained with usual care. They also noticed the reduction of these effects with longer interventions. Although the observed results were small and based on the short term, these authors sustained the potential importance of their clinical effects. In the analysis of these studies, the authors found that individuals during the active phases lost weight between months 3 and 12, whereas during maintenance stage they were going back to their baseline weight within 5.5 years.

Contento, Randall, Med, and Basch (2002) analyzed 265 studies whose main intervention was based on nutrition education published between 1980 and 1999, targeting different populations. These authors found fewer education interventions, defined as "any set of learning experiences designed to facilitate the voluntary adoption of eating and other nutrition-related behaviors conductive to health and well-being" (Contento et al., 2007, p.3), targeting younger and older adults than children (35\% vs. $85 \%)$. According to these authors, studies targeting this population have mostly been behaviorally focused with the goal to decrease the risk of chronic disease and to promote healthy eating patterns. In order to measure these behaviors, these studies have used a variety of systems ranging from a 24-hour recall to a 7-day food record. These authors noticed the need of evaluation measures appropriate to the purpose, duration, and power of the interventions. They also noticed the need for adequate validity and reliability of the measures in relation to both the outcomes and characteristics of the target audience. Physiological measures are often preferred because they are most directly related to health outcomes, but they have been found to not always be adequate to the typology of intervention 
they were used in. Based on the amount of evidence of the effectiveness of behavioral based nutrition education, changes in the dietary behavior became the most used outcomes, regardless the lack of standardization of the concept "behavior" related to food consumption (Contento et al., 2002).

Wu et al. (2008) review a series of eighteen studies from a meta-analytic perspective. The criteria to include these studies in their work were: the fact that they were randomized trials comparing a composed intervention of diet plus exercise and an only diet-based intervention. These studies need to be without any specifics in terms of the type of intervention, administered simultaneously, with an identical dietary program. In conclusion their duration had to be at least 6 months. Among these studies, 5 were based on less than 1 year long weight loss intervention, 11 on 1-1.9 years long weight loss intervention, and 2 on a 2 (or more) years long intervention. The mean age of the samples of these studies ranged from 36 to 55 years. Ten studies considered both men and women, whereas three included only women, and four only men. The dropout rates for these interventions were all but one lower than $30 \%$, without showing major differences between studies whose intervention was based on exercise and diet and those only focusing on diet. In their study these authors found that intervention integrating exercise and diet produced greater long-term weight loss overall in interventions longer than one year. Reaching long-term effects in interventions is not always easy and often researchers have found that the main reason for it is the lack of adherence. It has also been found that longer the intervention, for example using frequent follow-ups, reminders, etc, longer will be the effect. It was also found that, although both types of interventions created effects in weight loss, adding exercise to diet produced bigger effects, beside also producing other health effects. Similar results have been found by Curioni and Lourenço (2005) in their review of literature of randomized clinical trials 
looking at the effectiveness of combined exercise and dietary interventions. These studies focused on starting weight loss and their maintenance on the long term among overweight and obese people. This meta-analytic work included only six studies that directly compared intervention exclusively based on diet and interventions structured on a combination of diet and exercise. Samples of these studies had a mean age that ranged from 21 to 65 years and whose size ranged from 40 to 127 individuals. Three of these studies were looking only at women while one only based on men and the last two looking at both genders. The including criteria of the studies analyzed by these two authors were that the participants of the studies were older than 18 , with a BMI higher than 25. Studies were considered if they included a follow up of at least one year, and they did not include any specifics for diet or exercise typologies as well as no prescribed weight loss after intervention was established. All of these studies had lengths of the intervention that vary from 10 to 52 weeks, and had a follow up period that ranged from 12 to 24 months. Among the single sample of these studies the dropout rate was lower than $20 \%$ of the sample, but one study that reached $24 \%$. These authors found that the intervention with the combination of diet and exercise were obtaining weight loss $20 \%$ greater than those including only diets $(-13.0 \pm 10.4 \mathrm{~kg}$ vs. $-9.9 \pm 9.6 \mathrm{~kg}, \mathrm{p}<0.063)$. In conclusion, this meta-analytic work found that the addition of exercise to diet in the intervention targeting weigh loss created bigger effects. In addition, it also found that in both groups of intervention many were the additional clinical beneficial effects obtained along with the weigh loss. No differences between the two types of studies were found in terms of weight regain on a long-term period. This result showed how an initial greater weight loss was hard to maintain in the long term, also because, although individuals changed their lifestyles at the beginning, soon they came back to their older habits. 


\section{Alcohol Use}

Because of the pervasiveness of alcohol use and misuse within the young adult population, colleges have put into operation many alcohol-related interventions to address many of the relevant factors related to college student alcohol consumption (Scott-Sheldon, DeMartini, Carey, \& Carey, 2009). In their study, Wechsler, Seibring, Liu, and Ahl (2004) surveying 747 institutions, which constituted a national representative sample of schools in terms of geographical location, competitiveness, size of school, and geographical distribution between urban and rural areas. In their results, only $15 \%$ and $17 \%$ percent of the schools considered alcohol as a major or minor problem, whereas more schools perceived alcohol as a problem in their institution (66\%), and only $3 \%$ of these schools did not consider alcohol as a problem. Bigger schools (more than 10,000 students) were more likely to consider it as a major problem $(26 \% ; \mathrm{p}<.01)$ than smaller $(<5,000$ students; $12 \%)$ or medium size schools $(5,000-10,000$ students; 22\%). Bigger schools, besides showing more concerns, were also more likely to offer alcohol education and to offer other services related to this problem, but were also less likely to ban alcohol $(16 \%, \mathrm{p}<.01)$, to restrict its consumption at specific on-campus events $(21 \%, \mathrm{p}<.01)$, to create alcohol free campuses $(15.9 \% ; \mathrm{p}<.001)$, and to provide alcohol free residence halls $(26.8 \% ; \mathrm{p}<.01)$. Bigger and public schools were more likely to receive funding by public institutions for their alcohol educational programs, and they had bigger investments to provide alcohol related services to students. Among the public schools 35\% received public funding, $12 \%$ received private funding, and $21 \%$ received funding from the alcohol industry.

Bigger schools, which considered alcohol as a major problem, were also less likely to consider their interventions and prevention programs successful $(72 \%)$ than those considering it a minor problem $(83 \% ; \mathrm{p}=.005)$. In conclusion, Wechsler et al. (2004) found that 4-year 
universities are continuing their efforts to respond to heavy drinking and binge drinking, even though the perception of this problem seems to have gotten smaller. Many studies tried to provide interventions targeting the reduction of alcohol use in this typology of population with a variety of results. Qualitatively, the analysis of individual based interview brought some mixed results supporting some of these interventions, but not others (Larimer \& Cronce, 2002). It has also been found that the most effective of these interventions are those addressing individual motives for change and/or modifying exaggerated normative perceptions (Scott-Sheldon, DeMartini, Carey, \& Carey, 2009).

Scott-Sheldon et al. (2009) in their meta-analytic work analyzed 34 randomized control trials published between 1980 and 2007, constituted by a sample of 54 different interventions and 8,569 subjects participating in the studies, with a average age of 19,78 y/o ( $\mathrm{SD}=1.79)$. All these studies used interventions within the 33 to 45 minutes including alcohol education, normative comparisons, and moderation strategies looking only at the first assessment. These authors found that alcohol interventions had a variety of results on the participants compared to their control. Some of these were related to alcohol related knowledge with an increase due to the intervention $(d+=0.25$, CI.95 $=0.16,0.34)$. Some of these results showed improvement of attitudes toward drinking $(\mathrm{d}+=0.19, \mathrm{CI} .95=0.10,0.28)$ and of the normative beliefs regarding local students $(d+=0.35$, CI.95 $=0.26,0.45)$, and national college students $(d+=0.31$, CI.95 $=$ $0.19,0.43)$. Other results instead were related to the intentions to reduce alcohol consumption $\left(d+=0.09, C_{.95}=0.02,0.15\right)$. The effect sizes of the impact of these interventions were all small to medium according to Cohen (1988).

These authors found that the most effective interventions in increasing the alcohol-related knowledge were those specifically tailored to the individual and/or group $(\beta=0.35 ; p=.02)$ and 
including values clarification $(\beta=0.54 ; \mathrm{p}<.001)$ or moderation strategies $(\beta=0.52 ; \mathrm{p}<.001)$, but not focusing on high risk situations $(\beta=-0.44 ; \mathrm{p}<.01)$. These authors also found that those interventions sampling more men were found more successful at improving intentions to reduce alcohol consumption $(\beta=-0.44 ; \mathrm{p}<.01)$. Besides these positive results, according to ScottSheldon et al. (2009), it is noticeable the lack of findings supporting the increase of self-efficacy. This result could be due to the absence of specific factors addressing the improvement of it, such as successful experiences related to the interventions.

In conclusion, these authors have found that individual tailored interventions, as other health behaviors-related tailored interventions, induce to change because they are more likely to be read, remembered, considered credible, understood and perceived as interesting, and for this reason are more effective in leading to increased intentions to reduce alcohol consumption. Individual interventions (face-to-face or via computer or email) are more effective in improving alcohol-related attitudes, and they are also more effective in including values clarification component. In this latter case, knowledge and attitudes can be improved eventually through values clarification exercises, which can help the individuals to reassess their attitudes. ScottSheldon et al. (2009) also found out how intervention stressing the negative/high risk situations potentially produced by alcohol were less effective, and hypothesized that it was due to the fact that college students were already aware of these, whereas those including moderation strategies seem to be more effective for this population. Interestingly, these authors found out that the most effective intervention were those addressing mostly men, and hypothesized that it could be due to the fact that since men are more likely to experience these type of problems, they might have more reasons to develop strategies to limit and improve their attitude toward to alcohol use. 
In their meta-analysis Carey, Scott-Sheldon, Carey, and DeMartini (2007) looked at 62 studies, published between 1985 and 2007, including 98 interventions and sampling 13,750 college student participants with a retention rate at follow-up of $75 \%$. The sample of almost equally distributed between men and women (respectively $53 \%$ and 47\%), mostly White (81\%) with an average age of 20.37 years $(S D=1.98$, range $=18$ to 26$)$. Based on the 39 studies reporting the class, the sample was made on $66 \%$ freshmen, and $17 \%$ sophomores. The majority of these studies was held in the U.S. (85\%), and was conducted in public universities (72\%) of large size $(>10,000 ; 89 \%)$. They included in their analysis only studies that were designed to decrease alcohol use or its consequences in college students. These studies had to be characterized by groups randomly assigned to the conditions of the interventions. They also had to include a control group and to report behavioral outcomes related to alcohol use or related problems. These studies were characterized by an average of 1.87 intervention conditions each $(\mathrm{SD}=1.05$; range $=1$ to 5$)$, mostly involving face-to-face intervention delivered by a facilitator to an individual or group (70\%), and a minority delivered by computer (22\%) or in a combination (7\%). Forty-four percent of these interventions were delivered to groups, whereas $30 \%$ was delivered to individuals, and most of them were informed by theories (82\%) and guided by manuals (61\%; respectively present in $66 \%$ of the face-to-face interventions, $46 \%$ in the computer delivered interventions, and $57 \%$ of the combined delivered). Few of the factors characterizing these interventions were alcohol/BAC education (73\%), normative comparisons (56\%), and feedback on consumption (49\%). Some of these interventions also included moderation strategies (43\%), feedback on problems (37\%), goal setting (35\%), feedback on expectancies and/or motives (34\%). These authors identified two categories of outcomes: alcohol consumption outcomes and alcohol-related problems. The first category, the alcohol 
consumption outcomes, included (a) quantity of alcohol consumed over a period of time, or in a specific occasion, and per drinking day; (b) maximum quantity consumed in a setting; (c) frequency of heavy drinking and (d) frequencies of drinking days; (e) peak and/or typical blood alcohol concentration (BAC); and (f) composite alcohol consumption. The second category instead was operationalized with the use of a multiple item scales.

Based on the length of the follow up, the authors found a significant variety of results in many of the described categories. At first, at the immediate follow up, it has been observed that a reduction of the participants' quantity of drinking $\left(\mathrm{d}+=0.19, \mathrm{CI}_{95}=0.07,0.32\right)$, frequency of heavy drinking $\left(\mathrm{d}+=0.17, \mathrm{CI}_{95}=0.03,0.31\right)$, and peak BAC $\left(\mathrm{d}+=0.41, \mathrm{CI}_{95}=0.26,0.57\right)$ compared to those in the control groups. These individuals, at the short-term follow-up (based on 4-13 weeks post-intervention), showed a reduction in both the quantity of drinking $(d+=0.13$, $\left.\mathrm{CI}_{95}=0.06,0.19\right)$, in the quantity for specific time intervals/drinking days $(\mathrm{d}+=0.13, \mathrm{CI} 95=0.05$, $0.21)$, in the frequency of heavy drinking $(d+=0.18$, CI95 $=0.10,0.26)$, in the peak BAC $(d+=0.13$, CI95 $=0.04,0.21)$, and in the alcohol-related problems $(d+=0.15$, CI95 $=0.08,0.21)$. Similar results, although a little smaller, have been obtained at the intermediate follow-up (14-26 weeks post-intervention), and even smaller results were found at the long-term follow-up (27195 weeks post-intervention). At the middle-term follow-up, the intervention participants decreased their quantity of both alcohol consumed $(d+=0.11$, CI95 $=0.02,0.20)$ and alcohol for specific time intervals/drinking days $(\mathrm{d}+=0.19, \mathrm{CI} 95=0.08,0.31)$, and frequency of heavy drinking $(d+=0.11$, CI95 $=0.01,0.22)$. They also experienced a reduction of the amount of alcohol-related problems $(\mathrm{d}+=0.22, \mathrm{CI} 95=0.12,0.32)$ compared to participants constituting the control groups. At long-term follow-up (27-195 weeks post-intervention), instead, only the 
frequency of drinking days $(\mathrm{d}+=0.16, \mathrm{CI} 95=0.03,0.30)$ and alcohol-related problems were reduced $(d+=0.14$, CI95 $=0.06,0.22)$.

This meta-analytic work showed how interventions were more successful at reducing alcohol-related problems at short-term follow-up when the sample contained more women ( $\beta$ $=0.56, p=.01)$ and when the interventions were delivered individually $(\beta=0.28, p<.001)$ or inperson $(\beta=0.17, p=.01)$. In terms of content of the intervention, it shown how the most successful interventions were those in which motivational interviewing techniques were used ( $\beta$ $=0.21, \mathrm{p}<.01)$. Other successful interventions were those whose provided normative feedback $(\beta$ $=0.17, p=.01)$, feedback on expectancies and/or motives for drinking $(\beta=0.27, p<.001)$, or a decisional balance exercise $(\beta=0.17, \mathrm{p}=.05)$. The intervention targeting heavy drinkers $(\beta$ $=-0.34, \mathrm{p}<.001)$ or any other at-risk group $(\beta=-0.14, \mathrm{p}=.05)$ were found less successful. The same results also occurred for those interventions delivered via computer/mailing $(\beta=-0.22$, $\mathrm{p}<.01)$ and for those whose content included an expectancy challenge exercise $(\beta=-0.20, \mathrm{p}<.01)$ or some sort of skills training $(\beta=-0.28, \mathrm{p}<.001)$.

In summery, this meta-analysis produced three major findings: (a) individual intervention for college drinkers reduced alcohol use; (b) they also reduced alcohol-related problems with a range of reduction depending on the sample and the type of intervention used; (c) the differences between the intervention and the control participants diminished overtime. Based on the first finding, it seems that those students who received the educational intervention engaged successively in less extreme drinking behaviors. These findings also show how alcohol risk reduction interventions are effective in reducing quantity and frequency of drinking, as well as in reducing the alcohol related problems that extreme drinking behaviors carry with them. These results were found characterized by more effectiveness when more women were involved in the 
sample, who normally experience less alcohol-related problems than men, while they were less effective when targeting heavy drinkers or a high-risk group of individuals. This study also shows how the magnitude of the effect of these interventions diminishes over time from the short-term to the middle and to the long-term follow-up.

Moore, Soderquist, and Werch (2005) showed how a binge drinking prevention program specifically addressing college students, and delivered through the Internet, was feasible and efficient. These authors showed how there were no significant differences between the results found in studies addressing this type of program using more traditional delivery system and those using the Internet. In their study, the authors found that students were very comfortable in using the Internet, and this use was facilitated by the tools, such as an email account and internet access provided by the university. In terms of the study, the authors found out that the intervention was much more easily implementable and it was much faster to deliver information to the participants. Participants rated the internet intervention the same as the print intervention and based on the advantages offered by the internet intervention, it is possible to sustain the use of this delivery tool.

Agostinelli, Brown, and Miller (1995) found out that a simple no-face-contacted-based feedback, based on social norms, could be beneficial for heavy drinkers. In their sample they compared heavy drinkers receiving the feedback and those who did not receive it, and they found that feedback participants had greater reduction in weekly consumption and typical intoxication levels than the control group individuals.

\section{Multi-Behavioral Intervention}

Marcus et al. (2006) found out that many studies included multiple behaviors change intervention, mostly addressing PA and diet/nutrition, although other health behaviors have been 
included. Besides the positive short-term results of these studies, more research is needed to clarify the aspects of these multi behavioral studies.

In their reviews of studies focusing on PA, nutrition, or both, Wilcox, Parra-Medina, Thompson-Robinson, and Will (2001) found that intervention effects are not "diluted" when addressing more than one behavior concurrently. These authors found that combined interventions, focusing PA and nutrition, had similar effects than those addressing single behaviors.

Although there is still the need for further study, Michie, Abraham, Whittington, and McAteer (2009), in their meta-analysis, focusing also on multi behavioral change interventions, found that self-monitoring behaviors was a very useful technique for increasing the effectiveness of intervention targeting healthy eating and PA. The effectiveness of this technique was even more if it was involved in a system of techniques, prompting intention formation, specific goal setting, the review of behavioral goals, and providing feedback on performance. These authors also found that the number of behavior change techniques used did not enhance the effectiveness of the intervention, hypothesizing that the quality and fidelity of the intervention could be compromised by the use of many different techniques. According to Keating et al. (2005) PA and dietary intake are the two main factors supporting health. These behaviors are those that are normally addressed by the majority of the multi-behavioral interventions in focusing on weight loss/gain and its potential negative consequences. There are many studies and meta-analytic works proving the effectiveness of multi-behavioral interventions, mainly based on weight loss, but useful to prove the usefulness of a multi-behavioral approach of this type of strategy.

Curioni and Lourenço (2005) in their meta-analytic work found that interventions coupling up diet and PA/exercise were more effective than the one only using diet as strategy to 
fight weight gain. In addition they confirmed that engaging in PA as addition to a diet can also produce the already described long series of clinical benefits related to obesity and inactive living. Along with the same type of findings, Miller, Koceja, and Hamilton (1997) analyzed a sample of 493 studies (out of the original 700) published between 1969 and 1994. The including criteria of this work were mainly the fact that the participants were overweight $(120 \%$ of their ideal body weight, $\mathrm{BMI} \geq 27$, body fat $\geq 30 \%$ ); that they were 18 years old or older, and that outcomes, in terms of weight change, had to be expressed in numerical values. The sample size of these studies ranged from 3 participants to 2,869 , with an average age ranging from 18 to 68 years old. Twenty six percent of these studies focused on men, $46 \%$ on women, and $28 \%$ were targeting both genders. In terms of Effect Size, these authors found that the combination of PA/exercise (E) and diet (D), compared to those including only diet or PA, was the most effective in both weight loss (respectively effect sizes are 5.5 $\pm 0.7 \mathrm{ED} ; 5.1 \pm 0.5 \mathrm{D} ; 2.1 \pm 0.5 \mathrm{E}$ ) and fat loss (respectively effect sizes 7.9 $\pm 1.6 \mathrm{ED} ; 5.5 \pm 1.2 \mathrm{D} ; 2.1 \pm 0.7 \mathrm{E}$ ). These authors also noticed that although the differences in weight loss among the different interventions, all of these strategies offered approximately the same results in terms of one year maintenance. Specifically, $70 \%$ of the weight loss in interventions addressing only exercise were maintained versus the $73 \%$ of the weight loss maintained by the combined interventions and those only including diet. An interesting finding was the fact that interventions targeting both behaviors produced greater change in body composition, with a decrease in both percentage of body fat (7.4 $\pm 2.5 \mathrm{ED}$; 4.8 $\pm 0.8 \mathrm{D} ; 3.0 \pm 1.1 \mathrm{E})$ and in BMI $(3.7 \pm 2.0 \mathrm{ED} ; 2.2 \pm 0.5 \mathrm{D} ; 0.7 \pm 0.9 \mathrm{E})$.

Sustaining the usefulness of a multi-behavioral approach, Kelly and Melnyk (2008) reported how structured program focusing on PA, nutrition, and other behavioral skills seems to be effective in weight loss and in reducing cardiovascular risks. In their review, these authors 
looked at 17 studies that fitted in their inclusion criteria. Their sample was between 12 and 20 years old, not characterized by strong diversity, and mostly focusing on female participants. All the considered interventions provided information about nutrition and behavioral skills, and about half of them were focused also on PA. The major strength of this study is the fact that all the used studies were randomized controlled trials (RCT) which helped in establishing a causeeffect relationship between the outcomes and the interventions.

In their "Healthy PAC-CATS" project (Healthy Personal Assessment Challenge to Choose Activities for Today's Success), Newton, Kim, and Newton (2006) analyzed 168 freshmen students divided in two different conditions. These two conditions differed in the fact that one was characterized by a multi-behavioral intensive direct intervention and the other only by an educational intervention. These authors noticed an improvement in many of the targeted health behaviors, even though some exceptions were found in those students receiving only the educational intervention. Specifically these authors found that those individuals within the intensive change intervention improved more than the other students in all health behaviors, whereas the educational intervention students experienced a little increase in alcohol use. The statistically significant difference between the two groups have been found in "strength training" $(p=.044)$, alcohol beverage $(p=.010)$, positive thinking $(p=.009)$, creative problem solving $(p=.036)$, and stress impact $(p=.035)$. The intensive change intervention instead showed statistically significant changes between pre and post interventions in many health behaviors including: fruit and vegetables consumption $(p<.001)$, whole grain $(p=.033)$, low-fat of fat-free dairy $(p=.039)$, caffeine beverage $(p=.002)$, regular pop/soda $(p=.003)$, sweetened beverage $(p=.042)$, alcoholic beverage $(p=.019)$, relaxation techniques $(p=.078)$, positive thinking $(p=.001)$, creative problem solving $(p=.021)$, and stress impact $(p=.035)$. Besides the 
effectiveness of a multi-behavioral change intervention, this study offers many results that help to provide evidence of the effectiveness of targeting freshman college students showing how effective the intervention has been in changing behaviors such as the intake of fruit and vegetables, alcohol use, and the attitudes of these people toward those behaviors.

\section{Mediated Intervention}

According to Jenkins, Christensen, Walker, \& Dear (2009), there has recently been a growth of more accessible and low-cost interventions of this sort characterized by new and innovative delivering mean such as print, internet, telephone, and media.

Technological advances have and will impact many aspects of PA/exercise behavior changes science and application (Nigg, 2003). This is because also dietary (i.e. Kristal, Curry Shattuck, Feng, \& Li, 2000) and alcohol behavioral change (i.e. Cunningham, Wild, Cordingley, van Mierlo, \& Humphreys, 2009; Kypri, Saunders, Williams, McGee, Langley, Cashell-Smith, et al., 2004) have seen the birth of many applications influenced and/or led by new technologies. The use of new technologies has permitted to deliver these interventions to different populations, regardless of their location and with many other advantages. Jenkins, Christensen, Walker, and Dear (2009) sustain that these types of interventions have many advantages over the traditional interventions commonly implemented by health professionals. Some of these are the increased accessibility (Marcus et al., 2006), the potential cost effectiveness (van den Berg, Vliet Vlieland, \& Schoones, 2007; Napolitano \& Marcus, 2002), and the possibility of improved maintenance of gains at future follow-ups (i.e. Dishman \& Buckworth, 1996; Napolitano \& Marcus, 2002). According to Jenkins et al. (2009) these interventions could become extremely relevant in delivering population-wide interventions. Nigg (2003), based on his review of the literature in the PA realm, sustained that the link between technology and tailored interventions, besides 
increasing the amount of people to deliver the interventions to, was also useful in increasing the cost effectiveness of these interventions. This linkage also allowed for individualized feedback, which had been shown to be effective in PA promotion (Marcus, Emmons, Simkin-Silverman, Linnan, Taylor, Bock, et al., 1998).

Some studies have been looking at telephone based interventions (i.e. Albright, 2005;

Beers, Hohman, Slack, Sisneros, \& Woodruff, 2009; Green, 2002;), some were based on printed feedback (Marcus, Napolitano, King, Lewis, Whiteley, Albrecht, et al., 2007), and some others were internet-based (i.e. Harvey-Berino, Pintauro, Buzzell, DiGuilio, Gold, Moldovan, et al., 2002; Kosma, Cardinal, \& McCubbin, 2005; Plotnikoff, McCargar, Wilson, \& Loucaides, 2005). A brief review showing examples from each type is provided below.

\section{Tailored Interventions}

Within the behavioral change literature, it has been shown how those interventions addressing health behavior changes are more effective when providing individual and specific feedback than when structured on the "one-fits-all" mindset (Marcus, Nigg, Riebe, \& Forsyth, 2000). Often time, within the general literature about mediated and feedback proving interventions, the two terms "targeted" and "tailored", have been used alternatively to define the intervention, although they are characterized by different factors (Napolitano \& Marcus, 2002).

According to Kreuter et al. (1999), "targeting” refers to those interventions which define a population group, based on one general trait, and that for this reason can be influenced by the same message. The term "tailoring" origins from the idea that individuals, although part of the same population, are characterized by specific characteristics and motivations. Starting from this conceptual differentiation, it is possible to state that a given message targeted to a specific 
population may not be necessarily appropriate for a specific individual within it. They might not be able to offer and provide information about and for the individual specific characteristics.

An intervention is tailored when the participants are reached individually with an individual message, based on individual characteristics (Kreuter et al., 1999; Kreuter, Farrel, Olevitch, \& Brennan, 2000). Tailored interventions are customized to match select characteristics of specific individuals. They are generally consisting of a few main components: (a) the theory based assessment of the peculiarities of the individuals; (b) the preparation of the content, to match these characteristics and prepared in small segments, that often are coded and stored in a message library, ready to be used; (c) the preparation of the decision algorithm that provides the logic matching a particular message to the person's characteristics; (d) creating individualized messages, based on the specific union of the short segments, and (e) selection and preparation of appropriate strategies and channel of delivery for the message and delivery of it in an appropriate way such as via print, computer, telephone, and one-to-one (Lustria, Cortese, Noar, \& Glueckauf, 2009; Ryan \& Lauver, 2002).

\begin{tabular}{lcc}
\hline & Targeted & Tailored \\
\hline $\begin{array}{l}\text { Level of assessment needed } \\
\text { Personalization }\end{array}$ & Low/moderate & High \\
$\begin{array}{l}\text { Participant time/burden } \\
\text { Reachability }\end{array}$ & Moderate & High \\
$\begin{array}{l}\text { Level of uniqueness and } \\
\text { individualization }\end{array}$ & Low & Moderate/high \\
$\begin{array}{l}\text { Assumption that individuals who } \\
\text { share a characteristic are }\end{array}$ & High & Moderate/high \\
homogenous on other factors & Low & Moderate \\
$\begin{array}{l}\text { Cost (implementation) } \\
\text { Cost (development) }\end{array}$ & High & Low/moderate \\
\hline Notes: This table has been modified from Napolitano and Marcus (2002) & Low \\
& Low & Moderate \\
\hline
\end{tabular}

An example of the effectiveness of these interventions has been offered by Marcus,

Owen, Forsyth, Cavill, and Fridinger (1998) in their randomized work with 1,559 individuals. This study compared a self-help intervention tailored to the individual's stage of motivational 
readiness for exercise and a typical self-help exercise promotion intervention with randomized groups. The authors found that those individuals, among the intervention completers $(\mathrm{n}=903)$, who received the tailored intervention were significantly more likely to show increases (37\% vs. $27 \%)$ and less likely to show either no change (52\% vs. $58 \%)$ or regression (11\% vs. $15 \%)$ in stage of readiness. The limit of this study is the fact that, although the authors talked about tailored intervention, they developed five manuals for each of the five stages of the Transtheoretical Model (TTM). They distributed these manuals to the participants according to their scores at the baseline, make this a targeted intervention. They assumed that all individuals with similar readiness to change were characterized by similar needs, barriers, and understanding.

An example of a completely computer-tailored intervention is the study carried on by De Bourdeaudhuij, Stevens, Vandelanotte, and Brug (2007) addressing the decrease of fat intake, which delivered the intervention within a workplace. They structured the study with a quasiexperimental design randomly assigning 6 companies, with a sample of 337 individuals, to three different conditions: (a) the computer-tailored intervention, (b) the generic intervention, and (c) a no-intervention control. After having completed a validated baseline and posttest questionnaires, these individuals immediately received the personal feedback through the company's intranet. In a period of six months, the authors found the tailored intervention more effective in decreasing fat intake than the other two conditions $[\mathrm{F}=23.5, \mathrm{p}<.001$, generic intervention; $\mathrm{F}=28.1, \mathrm{p}<.001$, no intervention].

Ryan and Lauver (2002), in a literature review, compared tailored interventions and standard informational interventions. They found that among their sample, the tailored 
interventions were perceived more personal and relevant. These interventions were also better understood, read and remembered; and overall they were more effective.

Based on the fact that tailoring interventions need to recollect and use a very big amount of information, often, some technological aid, such as some electronic devises or software, are required to manage this information. Software programs can be used to store the message library and to execute the decision algorithm, characteristic factors of a tailored interventions (Ryan \& Lauver, 2002). In conclusion, as suggested by Lustria et al. (2009), tailoring is a communication strategy characterized by its being multi-dimensional and aimed at increasing the perceived personal relevance of health messages. Computer-tailoring, based on the collection and assessment of the participants' characteristics and on the use of evidence-based decision rules, enhances the creation and development of strategic individualized health feedback (Lustria et al., 2009). Always based on the assessment of the individuals, a feedback is oftentimes part of the intervention and can be implicit or explicit. Explicit feedback summarized the results of the assessment, as a sort of diagnosis, and the interventions are structured on the shared assessment of the individuals' characteristics. An implicit feedback, instead, is constituted by the intervening tailored message, without the statement on the detected characteristics (Ryan \& Lauver, 2002).

Lustria et al. (2009) conducted an extensive search for computer-tailored online behavioral health intervention studies published in English from 1996 to February 2007. Among the originally found 503 studies, a total of 30 online behavioral interventions, clearly reporting computer-tailoring methods, were selected for this review. Mainly these studies covered few of the most relevant health areas: nutrition and diet $(n=10)$, PA $(n=7)$, alcoholism $(n=3)$, and smoking cessation $(n=7)$. On top of these, there were three other studies, one about encopresis, on about eating disorders, and one focusing on general risk behaviors. The majority of these 
studies focused on risk prevention (e.g., obesity, cardiovascular disease), cessation (e.g., alcohol abuse and smoking), and health maintenance (e.g., nutrition, diet and exercise), plus one dealing with disease management and treatment (e.g., encopresis). In this study, these authors found four different ways commonly used to customize messages' content: health behaviors, stages of change, risk factors, and information needs. Among these the most used were health behaviors and stages of changes.

They found that online computer-tailored behavioral interventions were placed in a continuum in terms of implementation and level of sophistication, and they went from computerassisted risk/health assessments to computer-tailored content/customized health programs. The first end is characterized by typically short-term interventions with individualized feedback immediately provided online or sent by email later. The automation of these systems allows an anonymous and quick assessment facilitating the provision of non-intrusive, but individualized, feedback and potentially increasing the attention paid to these messages. It has been found preliminary evidence of the efficacy of short-term tailored interventions for health behaviors. At the opposite end, there are computer-tailored content and customized health programs, more complex and generally characterized by longer strategies. This type of interventions allows patients to access the program multiple times, better targeting those behaviors that may require longer interventions to overcome barriers to change, as in case of multiple behaviors.

Lustria et al. (2009) found that computer-tailored health interventions were typically delivered via four major channels: print, CD-ROM-based multimedia applications, computer, or the Internet. Among these, web-based delivered interventions offer the most diverse group communicating means, such as e-mail, print material, online newsletter, audio narration, video 
quizzes and games, and chat. These authors sustained that there are three main strategies to develop customized and individualized messages: personalization, feedback, and adaptation.

Personalization refers to the specific information gathered during the assessment, which can help in increasing the meaningfulness of the message, offering the idea that the message has been specifically developed for the person. Feedback, instead, includes recommendations based on an expert assessment of the individuals' needs and characteristics related to the behavior of interest. Adaptation, also called content matching, creates pertinent content packages for the individuals based on the assessment and on the targeted behavior norms. It needs to analyze the individual responses and understand what type of message would be more effective for a specific participant while matching consequentially the right content. These tailoring mechanisms are normally used in combinations, and the use of feedbacks is the most commonly used in the majority of the studies (Lustria et al., 2009).

Dijkstra and DeVries (1999) distinguished five different types of adaptation of the tailored message with different effects. These different types are; less redundant information, more relevant information, avoiding defensiveness which enhance attention, more information which creates more persuasive argument, and personal goal-setting which enhance selfregulation. These authors also distinguished five different types of feedback with each one having a different effect. They listed: (a) feedback on cognitive behavioral states; (b) ipsative feedback, which tend to enhance self monitoring; (c) normative feedback, which enhance social comparison, (d) positive feedback, which reinforce the desire states, and (e) feedback based in personal characteristics, which enhance curiosity and involvement. The latter type of feedback emphasizes personal tone and empathy, which enhance commitment.

\section{Telephone based interventions}


Eakin, Lawler, Vandelanotte, and Owen (2007) included in their review studies characterized by (only or mainly) interventions aimed at improving PA and/or dietary behavior in adults and delivered by telephone. They needed to present PA and/or dietary behavior outcomes. Randomized controlled trials were given priority for inclusion, but also quasiexperimental studies were considered. Among the original 879 studies, the total number of the studies fitting the including criteria was 26 . Sixteen of these studies focused on PA, 6 on dietary behaviors, and 4 looked at both behaviors. All these studies included a sample constituted by 9,237 individuals. Fourteen of these studies included healthy adults (primary prevention), while the other 12 were characterized by a variety of chronic conditions (secondary prevention).

Among those studies that focused on PA, 69\% showed effect sizes (calculated from eight studies) in a range between 0.24 and 1.19. Six of these studies reported on short-term $(<3$ months) outcomes, with positive results in two of them. Nine of them presented medium-term (3-6 months) outcomes, showing positive results in five of them. Six showed instead long-term (> 6 months) outcomes, with all of them showing significant improvements. Within those studies focusing on dietary behaviors instead, $83 \%$ of them reported significant improvements in dietary behavior with effect sizes (calculated from four studies) ranging from 0.40 to 1.10 . Two of these showed medium-term results, with both producing positive outcomes. Among the four of them structured on long-term results, three showed significant improvements. Only four studies presented a combination of the two behaviors. Three of them (75\%) reporting positive outcomes, two with significant improvements in PA and diet, and the last one instead only in diet. The effect size (calculated from one study) was 0.86 . Only eight of the 26 studies looked at the maintenance of treatment effects, and among them only four reported lasting effects. Across these studies the use of the phone-based intervention compared to another type of intervention 
was a common situation and only three of these studies presented an intervention only based on telephone. Among the "only phone" studies, only one showed positive outcomes. A combination of different interventions resulted in being more effective: $66 \%$ of those using phone plus print or phone plus face-to-face plus print, $75 \%$ of automated phone, $86 \%$ of multiple component, and $100 \%$ of phone plus face-to-face (Eakin et al., 2007). In conclusion these authors found that telephone based behavioral change interventions can be an effective tool, with the best results when used concurrently with another delivery system.

Ball, Salmon, Leslie, Owen, \& King (2005) showed positive results in using a telephonemediated intervention among mid-life and older Australian adults in addition to the print based intervention. In their study, the increased measures of self-reported global physical activity, moderate-vigorous intensity activity, and walking were found for both intervention groups: only print and print-plus-telephone. Within their sample, these authors found that participants in the print-plus-telephone group showed slightly higher levels of global reported activity and walking.

Kim, Pike, Adams, Cross, Doyle, and Foreyt, (2010), found that the addition of the telephone based counseling was beneficial in increasing fruit and vegetable consumption. These authors addressed a sample of 2,470 participants, randomized between self-help $(\mathrm{n}=1191)$ or self-help plus telephone counseling $(\mathrm{n}=1279)$ group. With their study they concluded that targeting multiple behaviors, using a theory-based behavioral change counseling technique, delivered through telephone-based system was valuable in promoting healthy diet and weight management.

\section{Interventions based on printed feedback}

Napolitano and Marcus (2002) focused their work on intervention mainly delivered through printed material. These authors showed some of the potential advantages that these types 
of interventions delivery provided (i.e. stimulation of self-initiated change, relatively low cost, large accessibility, reduction of time barriers, possibility to be reused and kept as a reference). They also showed how some of these could also be applicable to other informational technologies (i.e., computer expert system reports, e-mail, Internet). Normally "printed-based health education materials" refers to all those intervention structured on the use of massproduced brochures, booklets, newspapers, pamphlets, books, magazines, and personalized print or pamphlets as well (Kreuter, Strecher, \& Glassman, 1999; Napolitano \& Marcus, 2002).

Marcus et al. (2007) found that printed, as well as telephone, interventions enhanced the adoption of PA among adults. These authors also showed how those interventions relying on printed material could be effective also in maintaining this PA -related behavioral change for longer terms. These authors studied 239 previously sedentary, healthy men and women predominantly Caucasian (90.3\%), female ( $82.0 \%)$, and middle-aged ( $\mathrm{M}=44.5$ years). These individuals fit the including criteria by being healthy, between $18-65$ years old, and underactive (i.e., participating in moderate or vigorous physical activity for $90 \mathrm{~min}$ or less per week). These individuals agreed to be randomized in different groups. After the orientation session, the individuals have attended two sessions during which they were assigned to one of the three groups (Phone, Print, or control). The participants within the treatment groups received individually tailored messages, for a total of 14 contacts over during 12 months, and they were told that their goal would have been to increase their PA with the goal to meet or exceed the $\mathrm{CDC} / \mathrm{ACSM}$ recommendations. The primary outcomes of the interventions were collected at three time points: baseline, 6 months, and 12 months.

No statistically significant differences were found between the two groups on any of the variables at baseline. The authors found significant differences between the groups at 6 months 
$(\mathrm{F}=3.73, \mathrm{p}=.0254)$, with both the telephone group $(\mathrm{M}=123.32, \mathrm{SD}=97.64)$ and the print group $(\mathrm{M}=129.49, \mathrm{SD}=156.46)$. These authors also found much larger increase in minutes of PA per week in participants compared to the contact-control group $(M=77.67, S D=101.79)$. At the 12 month checkpoint, statistically significant differences have been found between print and telephone groups, favoring the print group, in terms of PA minutes per week however $(\mathrm{t}=2.72$, $\mathrm{p}=.0071$ ). Participants of the print group, compared to those constituting the telephone group, were not much better at six months $\left(\chi^{2}=1.18, \mathrm{CI}_{95}=0.62-2.24\right)$ in reaching 150 minutes of PA. Instead, they were better at the 12 months checkpoint $\left(\chi^{2}=3.55, \mathrm{CI}_{95}=1.76-7.16\right)$. These groups were constantly better than the control group. Besides showing the potential general effectiveness of the printed interventions, these authors also showed how this type of intervention can produce longer effects on the participants in their behavioral change.

\section{Internet-based interventions}

In terms of effectiveness of the use of internet in delivering behavioral change interventions, Brunsting and van den Putte (2006) suggested that online self testing and single piece of advice, tailored or not, can succeed in making precontemplators aware about their alcohol problem, motivating them to become contemplators. This idea goes along with the suggestion of the fact that screening in itself can persuade some individuals to think about a possible behavioral change (Freemantle, Gill, Godfrey, Long, Richards, Sheldon, et al., 1993). Although no similar findings have been found related to the other health behaviors targeted in this literature review, it is also plausible to consider the potentiality of similar effects also in delivering PA and diet internet-based feedback.

Kroeze, Werkman, and Brug (2006) reviewed intervention studies published from 1965 to 2004 . These studies were characterized by the fact that they were all focusing on computer- 
tailored intervention, defined as "if the tailored advice was generated through a computerized process" (p.206), and easily detectable from other potential interventions considered in the study. Another characteristic of these studies was the fact that the information provided with the feedback had to be delivered in a "non-personal" way (printed format, direct interaction with computer, or other media device). These studies needed to address PA or nutrition behaviors, as primary prevention, defined as "the initiation of lifestyle or behavioral changes to prevent the onset of chronic diseases in apparently healthy participants" (p. 206). These studies needed to be only randomized controlled trials with pre-test and post-test, and published only in English. Out of the 693 originally found studies only 30 fit these criteria: 11 focusing on promoting PA, and 26 on dietary behaviors. Two publications in both groups addressed the same study and in several studies these two behaviors were addressed together with others. All the PA studies addressed the promotion of PA, whereas 20 among the nutrition studies looked at decreasing fat intake, five studies at fiber intake, 1 study at calcium intake, and 2 studies at weight loss. All interventions gave tailored feedback on the current behavior to the respondents. In some cases there were also feedbacks related to other variables (i.e. awareness of performance and selfefficacy). Mainly, these interventions were delivered by means of computer-tailored letters, pamphlets, or brochures. The majority of the interventions used single contacts, including one tailored feedback. Five of these study addressed PA and thirteen addressed dietary-change interventions providing multiple feedbacks. These authors sustained the relevance and effectiveness of computer-tailored nutrition-education interventions was relevant, regardless the small ESs obtained. The effectiveness of these strategies was sustain also besides the fact that these studies also provided the effects of the intervention based only on short and medium terms, with more findings relevant to dietary behaviors than PA. They also sustained that computer 
tailored interventions can be considered as promising means to promote healthy diets and possibly PA. This suggestion was based on the idea that, even if small, when some effects can be reached in the population at large, from a public health perspective the potential effects, it can be significant.

Vandelanotte, Spathonis, Eakin, and Owen (2007), in their systematic review, found modest evidence of the effectiveness of website-delivered PA interventions. They found around a half of their targeted studies reporting significant positive changes, with small EFs. These studies provided effects produced by the intervention only reliable on the short terms, with a clear decrease in efficacy with longer terms, when follow-ups increased. Although these findings seem to not be too positive, some of their studies instead showed some positive evidence in terms of maintenance. These authors analyzed 15 studies, starting from 512 originally found publications, characterized by sample sizes ranging from 13 to 2121 . Two thirds of these sample were women, and they have an attrition rate ranged from $7 \%$ to $69 \%$. The inclusion criteria characterized the studies by them being focused on website-delivered intervention to improve PA. This also needed to be one of the outcomes, but did not have to be the only or primary study outcome.

Some of these studies clearly structured their intervention on one or more theories, mainly the Social Cognitive Theory, the Transtheoretical Model, and the Theory of Planned Behavior. Eight of the fifteen websites delivered found positive behavioral changes, and in five of them it was possible to calculate the ESs that resulted in a small mean of ES of 0.44. Six out of the ten studies reported short-term outcomes, four of the eight reporting medium term, and two of the five reporting long term outcomes found positive changes. Eight of the fifteen studies also had an initial face-to-face phase to explain the use of the website delivering the intervention. 
Four of these studies had positive PA outcomes compared to the seven that lacked of this initial phase. The findings indicate that adding a face-to-face contact previous to the beginning of the intervention could potentially increase the effectiveness of the intervention. In concluding their work, these authors reported to have found consistency in the recommendations provided in these studies. They supported the increase of interactivity of these interventions in order to enhance engagement and retention of participants, even though their own results did not strongly support the effectiveness of this delivering mean. Along with this suggestion, the authors also reported the presence of numerous suggestions indicating the usefulness of computer tailored interventions set in the website delivered intervention. Their scope would be to increase the interactivity and consequentially enhance participant retention and outcomes (Vandelanotte et al., 2007).

\section{The Use of Internet within the College Population}

The use of Internet affects the daily life of the U.S. population and the entire U.S. economy (U.S. Department of Commerce, 2004). This effect increases in importance overall in the last few years with the beginning of the broadband technology. This recent expansion of the Internet and its use is also evident also in the new ways these tools have been used in communicating, in seeking for information, for entertainment, and as transactional tool. In this report, it is evident how the communication aspect of this technology still represents the most common task performed with it. This new tool is commonly used by people of all ages and both genders, with only few little difference in duration, type of use, location, scopes, and etcetera (Thayer \& Ray, 2006). The U.S. Department of Commerce report shows that $70.7 \%$ of the general population between 18 and 24 years old uses the Internet, and that among them the $86.7 \%$ of those in school were Internet users. Although this population mostly uses the Internet 
as a communication tool, it is also used as a source of information. Hargittai and Hinnant (2008) found that the people seeking health information online were younger than those looking for that information off line.

According to Subrahmanyam and Lin (2007), the Internet has become extremely important in the daily life of adolescents for instrumental purposes (i.e. school, information gathering, etc). This growing importance and popularity also made the Internet become one of the main communication means for adolescents. It also assumed a very relevant role in the social context of their development. These authors, in their 192 participants sample did not find any differences in terms of gender or ethnicity regarding the possibility to have access to Internet. Instead Hargittai and Shafer (2006) found differences of gender in terms of frequency of use and perceived internet skills noticing how men were higher in both perspectives than women. Odell, Korgen, Schummacher, \& Delucchi (2000) found that males and females both were characterized by an extensive use of the internet with only a little difference in terms of time spent on the Internet. This finding confuted the gender gap sustained by older literature regarding this aspect of the internet use, but finding at the same time differences in the way internet is used by the two genders. Weiser (2000) found that men have the tendency to use the Internet more commonly for leisure and entertainment, and women tend to use it more often as interpersonal communication tools and for gathering educational and academic information.

Gordon, Juang, and Syed (2007) have noticed the increased presence of the Internet in people's life, noticing that college students are deeply involved in its use. Jones (2002) sustains that by the age of 18 , almost everybody has been online, and found that $86 \%$ of the college population has gone online. Jones also found that $85 \%$ of these students own a personal computer with $66 \%$ of them having at least 2 email addresses. This information brought to 
consider the Internet as a fundamental part of their academic and personal lives (Ascribe Newswire, 2002). In his study on the 2,054 students, sample comparable to a representation of the national college population, Jones (2002) offered a description of the college population in terms of its use of Internet. This study found that about $75 \%$ of his sample used the Internet for four or more hours per week and that $12 \%$ uses it for more than 12 hours per week. All these results show how relevant and present computers and their functions are within this population of emerging adults living the college experience.

Gordon et al. (2007) studied the presence of the Internet in colleges and universities, finding that almost all college students are already online when starting college. They showed how most of the U.S. colleges and universities offer internet-accessible computer laboratory constantly available day and night to their students, sustaining once again the considered relevance of both computer and the Internet in the college life. Although Odell et al. (2000) found that students living on campus spend an extensive amount of time using the Internet. They also provided a novel result showing that there is not detrimental effect of the Internet in terms of time spent studying, possibly because it is also used for academic purposes. Thayer and Ray (2006) found a strong increase of the use of the Internet in the academic life of the young adults. These authors also speculate that this necessity in the academic life has permitted these younger individuals to better integrate this instrument in their personal and social life.

Jones (2002), with his sample, found out that the use of the Internet mainly as a social tool to make friends and communicate with them is still the preferred use of it for the young adults. About one-fifth of the sample uses the Internet as a friendship-making tool, about onethird uses it to maintain and develop friendships started with people met on campus. The study is dated 2002, a year before the spread of the use of Internet social networks, which increased the 
typology of computer mediated social interactions. With the advent of these new tools the amount of time spent on the Internet could increase also because of the increase amount of potential applications involved in these systems. The study itself is limited to the use of emails and instant messaging which are definitely a useful tool to maintain social relationships, but that are also characterized by limitation, overcome by the Internet based social networks.

\section{The Expert Systems}

According to Nigg (2003), one method to deliver this individualized feedback is represented by the Expert Systems. They are software systems that mimic the reasoning of a human expert and provide computer generated progress reports built on intervallic assessments (Nigg, Riebe, Rossi, Velicer, \& Prochaska, 1999).

Expert Systems, as their name states, is software able to transform the computer in an expert in a certain field. Expert Systems is computer software derived from the branch of computer science research named Artificial Intelligence (AI). The scientific goal of the AI is to understand intelligence by developing software able display an intelligent behavior (Engelmore\& Feigenbaum, 1993). According to Engelmore and Feigenbaum (1993), with the term intelligence it is possible to refer to many different cognitive skills. These authors also sustained that the AI, although generally addressing all of these skills, has shown the biggest progress in the area of problem solving. Every software achieving expert-level competence in solving problems, using a specific knowledge related to a particular task, is labeled knowledge-based or expert systems. Often the two terms, expert systems (ES) and knowledge-based systems (KBS), are used together to represent the most extensive application of AI. Task domain is the area of human reasoning that the ES needs to emulate. With "task" it is represented some goal-oriented, problem-solving activity (i.e. diagnosis, planning, scheduling, etc.), whereas with the word 
"domain" it is represented the area within which the task is being performed (health, law, transportation, etc.).

ES is software where an experience is memorized allowing an interaction between individual and machine. The knowledge, on which the ES is based, can be produced by the experience of an expert or by the analysis of previous data or by the integration of both these sources of knowledge. The starting knowledge on which the ES begins can be improved and enlarged using the new data insert by new users. This data could update the previous information in the decision making process, evolving the entire process. The KBS does not necessarily rely on the presence of previous data, but relies on theoretical constructs and expertise. When the system is started to be used, it can produce a data-bank to use in the future to improve the system or as comparison (Bandini, personal communication, December, 2009).

The branch of science whose main goal is to develop an ES is known as knowledge engineering. The main task of a knowledge engineer is to be sure that the computer has all the knowledge necessary to face and solve the proposed problem. The engineer also needs to ensure that the computer will be able to use it efficiently by selecting the appropriate reasoning methods (Engelmore\& Feigenbaum, 1993).

The ES found its knowledge on previous performance, on theoretical knowledge or on both. The interactivity of the ES is provided by the fact that, based on the answers provided by the individuals to certain questions, and the already stored information, it will provide a specific feedback to the task. This feedback will be tailored on the individuals' specifics and characteristics. ES are characterized by a variety of different way to disseminate the information produced by the interaction individual-machine. These different ways are the results of the 
assessment and the processing role of the decision making process, structured previously by the researchers (Nigg, 2003).

According to Nigg et al. (1999) these delivering systems have some advantages and disadvantages characterizing their use to deliver health behaviors interventions. These disadvantages are summarized by these authors with few concepts that include the potential participants' apprehension toward the use of computers, the required high technical expertise, the higher developmental and instrumentation cost, and the need of an explicit model of decisionmaking and already present data. Knowledge based systems can avoid this last need, being built on theoretical constructs and expertise, therefore not needing pre-existing data. KBS, instead, produce, through the interaction individuals-machine, a data-bank to use in the future to improve the system or as comparison. The list of potential advantages is divided in "theoretical" and "practical" advantages. The "theoretical" advantages were represented by the fact that all these systems use relevant information and pay attention on the individual characteristics. These systems count on a consistent group of decision rules, which allows also the possibility to replicate the results. These systems are also characterized by their being able of integrating the behavior assessment and the developed intervention, being cost affective, once already built up and structured. In terms of "practical" advantages they are flexible in terms of produced feedback and are cost effective. Their cost effectiveness is based on their needing low quantity and quality maintenance, their providing immediate feedback, avoiding delayed intervention and the use of other delivery systems (Nigg et al., 1999).

\section{Technical Characteristics.}

According to Engelmore and Feigenbaum (1993), an ES consists in two main parts: the knowledge base and the reasoning, or inference, engine. The first one includes two types of 
knowledge, the so-called factual and heuristic knowledge. The first is the knowledge of the specific task domain, which is usually largely shared, easily reachable on textbooks or journals, and commonly agreed upon by the "experts' in that particular area. The latter, the so-called heuristic knowledge, is much less rigorous, and more experiential; it is rarely discussed, and is largely individualistic. It is the one based on the well practice, on the good judgment, and on the plausible reasoning within the targeted area.

The so-called knowledge representation formalizes and systematizes the knowledge. This process is accomplished in different potential ways, such as the rule-based systems and the unitbased systems (Engelmore \& Feigenbaum, 1993). The rule-based systems are developed on a series of rules, which consist on an IF-THEN structure (also called a condition-action structure). The IF factor sets a list of conditions in some logical combination and the rule is relevant to reasoning developed if the IF part of the rule is satisfied. Only when this condition is satisfied the THEN factor can be concluded, or its problem-solving action taken. ES, whose knowledge is represented in rule form, are called rule-based systems. The unit-based systems are built upon the so-called unit (known as well as frame, schema, or list structure), based on a more passive view of knowledge. The unit is a collection of connected symbolic knowledge related to the entity that needs to be represented. Usually, a unit consists of a list of properties of the entity and associated values for those properties. These properties can be used to specify relationship among the many entities generally constituting a task (Engelmore \& Feigenbaum, 1993).

The reasoning engine includes the problem-solving model, and manipulates and uses the knowledge with the scope of forming a line of reasoning. The problem-solving model organizes and controls the process followed to solve the problem. One common problem-solving model involves chaining the IF-THEN rules to form a line of reasoning. This line of reasoning can go 
forward or backwards. This depends if starting from a set of conditions to move toward some conclusions, "forward chaining", or if starting from a known conclusion and move to find out the followed path, “backward chaining”. Based on the idea that more experience, and consequentially knowledge, an expert has, larger is the store of knowledge to look at in order to interpret and elaborate information. Along with this knowledge, also the amount of information used to elaborate appropriate solution to the targeted task (i.e. diagnosis, design, and analysis) increases. Based on these premises, it is possible to state that the most important aspect of any expert system is the initial knowledge and its ability to store it. The power of ES resides in their specific, high-quality knowledge related to the task domains (Engelmore \& Feigenbaum, 1993).

The final product of the ES looks like a chain of different modules starting from the knowledge provided by the expert and concluding with the information provided to the user. As in the image below, the expert provides the knowledge that enters in the Expert System Shell (general software to develop the ES). This knowledge will be acquired through a knowledge acquisition system, and passed through both the knowledge based facts and heuristics and the inference mechanism reasoning with uncertainty. Once elaborated the information, it goes into the explanation systems where the outcome is prepared to go to the user interface. This interface is able to present the answer to the problem to the user in a accessible manner (Nii, 1993).

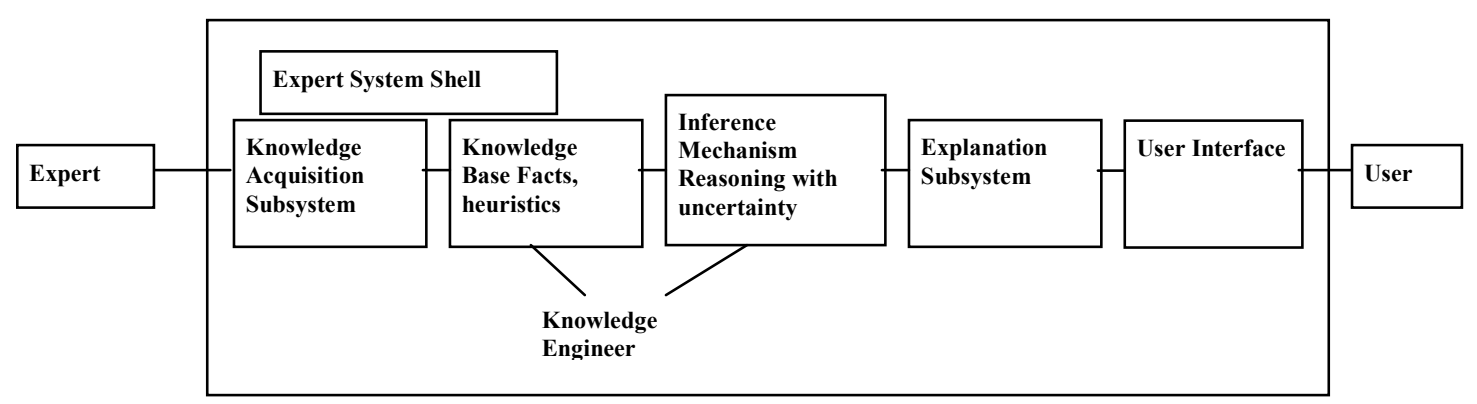

Note: Image modified from Nii, 1993 


\section{Expert Systems and Web-Based Computer-Tailored Health Behavior: Effectiveness of ES in Changing Health Behaviors}

According to Carron, Hausenblas, and Estabrooks (2003), ES, if delivered and used through the Internet, would allow participants to complete them from home or a community's computer, allowing them to obtain the results of their assessment immediately. It would also substantially cut down many of the barriers, such as time and accessibility to professionals. One of the strengths of this system is also the fact that, going along the development of new technologies, they can be delivered also through other devices.

The use of ES in delivering health behavioral change interventions has been proved to be successfully to promote physical activity (Marcus et al., 2006) and nutrition (Vandelanotte, De Bourdeaudhuij, Sallis, Spittaels, \& Brug, 2005). Vandelanotte et al. (2005) sustain that, although computer-tailored PA and nutrition interventions have been showed as generally effective, only recently these intervention reached total interactivity between participants and system. In previous works using this technology, the feedback was offered only after a few weeks, when the feedback was elaborated in laboratory and sent to the individuals. More recently a second generation of computer-tailored intervention was born, which permit a really simultaneous interactive computer-tailored intervention trend. These authors found out that interactive computer-tailored interventions were effective in modifying behaviors and that just one exposure to feedback was sufficient in creating this change. In their study, both interventions, simultaneous and sequential, created more improvement compared to no intervention.

Statistically significant differences $(p<.001)$ were found only in the decrease of fat intake with the simultaneous revealing to be more effective, whereas no significant differences were found in the level of PA. 
In terms of PA interventions, similar results have been found by Spittaels, De Bourdeaudhuij, Brug, and Vandelanotte (2007) in their research developed in six different worksites. Their sample was constituted by 526 individuals between 22 and 55 years old, and they were randomly grouped in three different conditions: (i) no intervention (177); (ii) onlinetailored PA advice with stage based reinforcement emails (174); (iii) online-tailored PA advice only (175). After a six month post-baseline questionnaire, no evidence of the higher effectiveness of computer-tailored intervention compared to an online standard advice was found. Although this finding, the authors found that this type of intervention was more appreciated and better used by the participants. Therefore, these authors stressed the fact that these interventions would have the possibility to be implemented with great benefit.

According to Neville, O'Hara, and Milat (2009), based on the findings of their narrative (no-data based) review of literature, studies based on computer-tailored dietary behavioral interventions showed positive outcomes. These authors also noticed that the efficacy of these types of interventions also depended on many other factors such as intervention quality, duration, exposure, intensity, use of theory, method of tailoring, and mode of delivery. These authors also recommended to use a combination of validated self-reports with more objective measures of behavior change. According to these authors the applicability of these interventions to real life, depends on the setting, the characteristics and representativeness of population sample that the intervention wants to target, and also on the methods used to recruit this population sample.

Strecher, Shiffman, and West (2005) studied the effectiveness of a web tailored intervention over a non-tailored intervention among nicotine patch users. They found that the first one was more effective than the second and that this type of intervention may offer a fairly 
higher-reach, higher-efficacy, lower-cost approach to health behavioral change. Based on this result, the authors sustained effectiveness of web-based tailored intervention.

In their review work, Lustria et al. (2009) concluded their study sustaining that the delivery through the Web of tailored programs to larger audiences, besides facilitating the implementation of these interventions, can also effectively enhance the access to the related benefits regardless of geographical and temporal limits. The use of Web environments also permits the developer of the intervention to include a richer variety of interactive components with the goal of enhancing the experiences of the participants. This situation would also support the achievements of the health behavioral goals for which the intervention was built. According to these authors, the use of specified tailoring criteria linked and coupled with a Web-delivered format could increase the level of acceptance of health information among the participants.

\section{TTM and TPB in Tailored Internet-based Health Behavior Interventions}

Some authors argue that the Transtheoretical Model [TTM] (Prochascka \& DiClemente, 1982; 1983; Prochascka, Norcross, \& DiClemente, 2006) is arguably the most commonly used theoretical framework in interventions focusing on health behaviors (i.e. Armitage, 2009). More specifically, other authors sustain that it is the most commonly used tailoring criteria in interventions focusing on nutrition, diet and exercise, and in those focusing only on PA (Lustria et al., 2009). They also found that TTM was often used to create tailored messages. TTM is very appropriate for interventions whose goals are behavioral changes depending on the stages of motivational readiness characterizing the individuals. This model has been used both in prevention and cessation programs (e.g., smoking cessation programs; programs that aim to improve diet, increase physical activity, and discourage alcohol use) (Lustria et al., 2009). 
The TTM can be considered as an integrative model of behavioral change (Velicer, Prochaska, Fava, Norman, \& Redding, 1998) created as an attempt to unify in only one model the many different theories and behavior change techniques (Armitage, 2009). It is constituted of different components summarized by Prochaska, Redding, Harlow, Rossi, and Velicer (1994a) in three categories: (i) stages of changes (precontemplation, contemplation, preparation, action, maintenance); (ii) dependent variables (decisional balance and self efficacy/temptation); and (iii) independent variables (ten processes of changes). This model was proposed as a framework to understand behavioral change characterized by the transition of the individuals through the five stages, characterized by different peculiarities (Prochaska \& DiClemente, 1983).

Precontemplative are those individuals who are not thinking about changing behaviors and are not aware of the consequences of these behaviors in terms of health. Contemplative are those who started to see the potential benefits of changing, but still engaging in the behavior. In preparation, instead, are those individuals who are preparing themselves and their social environment to change the addressed behavior. In action are those who actually changed their behavior. Only after maintaining the new behavior for over six months, these individuals can be considered being experiencing the maintenance stage (Armitage, 2009). Then there are the processes of change, strategies that people use to change their behavior or to protect their current behavior from relapse (Armitage, 2009). The processes of change are divided in two main groups: experiential processes and behavioral processes (Lox, Martin Ginis, \& Petruzzello, 2006). These two groups include each five of the ten processes of changes, which are consciousness raising, self-reevaluation, environmental reevaluation, dramatic relief, and social liberation. The behavioral process, instead, include self-liberation, counterconditioning, stimulus control, reinforcement management, and helping relationships. Each of those, conceptualized as 
independent variables, represents a different step toward a behavioral change and their usefulness is higher or lower depending on the stage it is experienced (Prochaska, Norcross, \& DiClemente, 2002)

The dependent variables are, according to Prochaska et al. (1994a), decisional balance and self-efficacy/temptations. Decision balance is the individual personal evaluation of pros and cons of the behavioral change. Instead, self-efficacy refers to the situation-based confidence that the individual experiences in terms of his or her ability to cope potential relapse-eliciting. Temptation is defined as the "intensity of urges to engage in a specific habit when in the midst of difficult situations" (p.40). This includes: negative affect or emotional distress; positive social occasions; and cravings situations (Prochaska \& Velicer, 1997).

Although this model has been used extensively within interventions addressing many different behaviors, such as diet, substance abuses, and PA (Hutchinson, Breckon, \& Johnston, 2008), some authors have started to discuss the real effectiveness of this model. In contradiction to the critics to this model, Hutchinson et al. (2008), in reviewing a theoretical model applied to practice, sustained that it is necessary to evaluate how faithfully this model has been applied in intervention-based studies, and they found that only in very few studies it has applied in all its facets consequentially losing its characterizing multidimensional aspect, and its total potentiality.

Some authors sustained that the stages of change are only a partial aspects of the model, an organizational construct, not providing any explanation of the behavioral change. They also add that it is only adding and considering all the facets of the model, in its entireness and completeness, that this model can assume an explanatory role (Bridle, Riesman, Pattenden, Sowden, Mather, Watt, \& al., 2005). 
Another characteristic to take into consideration planning an intervention based on the TTM is the fact that the relationship between stages and processes of changes varies depending on the specific behavior change targeted (Velicer et al., 1998). Being that this framework is very broad to potentially include many different behavioral changes, it might be important to look at the specific targeted behavioral change in order to build a behavior-specific model (Hutchinson et al., 2008).

The TTM has been found functional in terms of intervention planning. This framework allows the intervention to be targeted to the specific characteristics of the population within the specific stage (Armitage \& Arden, 2008). This classification could also help in tailoring even more specific and individualized messages to the single individuals.

Armitage (2009) in his review found that many studies brought to think to the existence of a two stages model. These two stages are: the first including precontemplation, contemplation and preparation and the second including action and maintenance. This idea was tested in the use of the Theory of Planned Behavior [TPB] (Ajzen, 1991) as motor of movement between one stage and the other. This theory explained very well the transition between most of the stages. Some difficulties were experienced in explaining the transition between the preparation and the action stages (Armitage Sheeran, Conner, \& Arden, 2004).

According to Ajzen (1991), the TPB proposes that behaviors are predicted by the intention of individuals. This intention is a function of the other three variables: attitudes towards to the behavior, the subjective norms regarding the behavior, and the perceived behavioral control of the individual on the targeted behavior. The perceived behavioral control has also direct influence on the behavior itself, without being filtered by the intention. The effectiveness of the TPB in predicting health behavior is generally recognized (i.e. Conner \& Sparks, 2005). 
Armitage et al. (2004) sustain that the use of decisional balance and self-efficacy as predictors of change, within the TTM, could exclude a series of possible different variables. To explain this, they sustained that the beliefs that strengthen the TPB are equivalent of decisional balance related to act a behavior. For this reason these beliefs are able to provide a summery of the processes associated with decisional balance. Ajzen (1998) also sustained that the perceived behavioral control and self-efficacy were synonymous. On these bases it is possible to sustain, as did by Armitage et al. (2004), that attitude (positive or negative evaluation of acting the behavior) and perceived behavioral control (extent of the perceived control of the individual on his/her ability to act the behavior) count for the TTM's decisional balance and self-efficacy. On top of these factors, the TPB also mediates effects of sociodemographic variables on health behavior and incorporates two constructs excluded by the TTM: the subjective norms and the behavioral intentions (Armitage et al., 2004). Ajzen (1991) considers the behavioral intention as representing how hard an individual wants to try and how much of an effort the individual is planning to put in order to act the behavior. Normally, the stronger the intention is, more likely the behavior will happen, but it is only related to those behaviors totally under the control of the individual volitional control. Subjective norms, instead, represent the perceived social pressure to act, or not, a specific behavior (Ajzen 1991).

According to Armitage et al. (2004) the TPB has been used consistently to predict health behavior because of its consistently supported predictive validity. Besides the limits of measurements, the TPB could be considered a useful framework to predict the transition among the different stages (Park, Lee, Hong, Kim, Kim, Kim, et al., 2009) addressing many different behaviors such as exercise (i.e. Courneya, Plontikoff, Hotz, \& Birkett, 2001; Park et al., 2009) and diet (i.e. Amirtage \& Arden, 2002). In many studies it has been found how the variables of 
the TPB increased along with the transitional process between stages of the TTM (i.e. Armitage \& Arden, 2002; Armitage et al., 2004; Courneya, 1995). Due to all these interconnections and the fact that the two theories seems complementary, TTM and TPB have been thought to be useful in planning an intervention able to enhance health behaviors with the goal to reach a healthier life style (i.e. Park et al., 2009; Vandelanotte et al., 2005).

In conclusion it seems that the integration of these two theoretical frameworks could be usefully adopted in the delivery of a behavioral change intervention addressing multiple health behaviors. This integration does not necessarily need to be a integrative approach of the two or a compound of the two, but it can vary depending on the intervention and its goal.

\section{The Use of Social Networks within the College Population}

Raacke and Bonds-Raacke (2008) sustained that this strong presence of the Internet in emerging adults' lives as a new communication tool, has changed the interaction styles among people. These authors also sustain that the majority of college students use friend-networking sites, such as Facebook and MySpace, for a good portion of their daily life both to communicate with friends and also to find old friends. These sites offer a new type of communication defined as idiosyncratic communication. These sites have become extremely popular among the college population, continuing to grow in numbers of individuals linked to them. They also keep increasing in type of features offered within the sites in order to increase the level and quality of communication among individuals through the website.

The use of these websites has increased in relevance and importance in these populations life and in their ways to build relationships (Valkenburg, Peter, \& Schouten, 2006). Social networking sites are very different, and they can differ from each other in terms of the type of relationship they focus on (i.e. dating, friendships, religious, interests, etc.). According to 
Valkenburgh et al. (2006), these are normally open or semi-open systems where everybody is welcome to open an account. In some cases instead, they just need to be invited by an existing member of the network. Each member introduces theirself to everybody through their profile, which contains descriptions and pictures. According to Chapman (2007), Facebook (and also other similar social network websites) is one of the most relevant phenomena within college students' lives since the Internet started to be a daily part of the college experience. This social network has deeply changed the communication among students and not only, with some examples of communication between students and professors (Recruitment \& Retention in Higher Education, 2006).

In their study, Raacke and Bonds-Raacke (2008) had a sample made up of 116 college students (53 male and 63 female) with an average age of 19.7 years (SD=3.7). Among their sample, $87.1 \%$ stated to have an account on Facebook or MySpace. Among this sample, $74.3 \%$ had both accounts, although Facebook was the most used (90.1\%). These authors did not find differences between men and women in terms of account ownership. They also found that only the $12.9 \%$ of their sample did not have any sort of account in a friend-networking site. The main difference between users and non users was age, with non-users significantly older than users, with a mean age of 19.05 and 24.80 respectively. This sample averaged 1.46 hours spent on their account and 1.10 hours spent on other's account per day, averaging around 3 hours per day spent on this websites, with an average of 4.19 log-ins per day. Men (44.9\%) were more likely than women $(23.1 \%)$ to use this type of website to learn about events $[\chi \mathbf{I}(1, \mathrm{~N}=101)=5.38, \mathrm{p}<0.05]$. Although these authors found some differences among genders comparing uses and gratifications for users, they also found that there were not main differences when analyzing the most popular uses and gratification. 
Krivak (2008) states that Facebook has over 60 million active users, being the sixth most trafficked sites in the U.S. The majority of this population of users is consist of college students, who mainly find in this website the original friendship making tool. These students also find through Facebook a way to gather information about many different topics, to spend their free time with its games, and to upload their pictures and watching movies.

These systems have also been found useful by students in other spheres of their lives, for example as a tool to gather information about future colleges. These systems have been found useful to make these choices, based on the students' perspective, with the goal to make a more accurate choice in terms of where to apply. These students also use this tool to look at where their friends are going to college, where they could study what they want, where there is the best professor student ratio $(\mathrm{T}, 2007)$. These systems have also been recognized in their more functional use by the institutions themselves recognizing that meeting their students where they already are would be the best way to stay in touch with them and communicate with them. The institutions also understood that to efficiently use this communicative tool, it was needed to learn help how to use it in the most efficient way with the goal of being heard by the students, for example keeping short and limited messages posted (Recruitment \& Retention in Higher Education, 2006).

West Virginia University already uses this online network system in a variety of ways. It is being used to contact students within the residence halls as outreach tool to contact future incoming freshmen students and alumni. Some examples of the use that WVU does of Facebook are those pages used as wellness groups (WellWVU FBpage) and departmental sources. This social network is also used in many other ways, for example as platform for many of the non- 
academic group based within WVU to outreach students within campus, such as Greek

Organizations and religious based group.

\section{The Rationale of Developing a New and Alternative Computer-Mediated Health \\ Behavioral Change Intervention Addressing College Freshmen Students:}

Based on the many different findings and on the numerous studies reviewed in this literature review, it seems appropriate to develop and deliver an intervention study targeting young emerging adults during their freshman year. It seems to be effective to develop this intervention using a web-based computer-tailored intervention delivered by the Internet and theoretically base it on the TTM and the TPB.

McTigue, Garrett, and Popkin (2002) addressed the necessity of an early intervention addressing health behaviors. This is because of the fact that health-risks are generally established during adolescence and young adulthood were then maintained during adulthood (McCracken, et al. 2007). For this reason, freshman college students constitute a well-targeted population to address with this type of intervention (Ferrara, 2009). This population could potentially find the most usefulness being addressed in their health behaviors, such as diet, alcohol use, and PA in order to identify the origins of, and eventually fight, the freshman year weight gain (Kasparek, 2008). The use of college campus resources and settings have been based on the fact that some authors (i.e. Ferrara et al., 2008; Nelson et al., 2008) considered the college experience and the campus setting itself a potentially natural setting for health-promotion interventions and efforts.

The choice of using Web-based computer-tailored intervention has been based on the fact that it has been found how college students, freshmen in particular are an "online population" (U.S. Department of Commerce 2004). This population is considered online because the high frequency of college students (72\%) accessing the Web (Anderson, 2001) and because of the 
fundamental role played by the Internet in their lives, both personally and academically (Ascribe Newswire, 2002). Based on these characteristics of this population, reaching those people through the Internet world could potentially increase the reachability and effectiveness of the intervention. Another cause of this choice has been the fact that computer-tailored interventions addressing health behaviors have been found effective in addressing health behavior as PA, nutrition, and alcohol (Lustria et al., 2009). The delivery of these interventions through the Internet and the use of ES-KBS (Carron et al., 2003; Nigg et al., 1999) has been proved as efficient in different works (i.e., Spittaels et al., 2007; Vandelanotte et al., 2005) within the realms of nutrition and PA. In top of this, many other authors successfully addressed other health behaviors (i.e. Strecher et al., 2005), but it has not been tested in a multi-behavioral intervention addressing concurrently PA, Diet, and Alcohol use at the same time. Overall this type of intervention has not been used to address this specific population within the same institution, yet. 


\section{References}

(2002, September 16). Internet essential to college life, study says. Ascribe Newswire: Health, 3-4. Retrieved from Health Source - Consumer Edition database.

(2006). How to make Facebook your new best friend. (Cover story). Student Affairs Leader, $34(4), 1-2$.

(2006). Using online networking to engage and retain students. (Cover story). Recruitment \& Retention in Higher Education, 20(3), 1-5.

Agostinelli, G., Brown, J. M., \& Miller, W. R. (1995). Effects of normative feedback on consumption among heavy drinking college students. Journal of Drug Education, 25(1), $31-40$

Ajzen, I. (1991). The theory of planned behavior. Organizational Behavior and Human Decision Processes, 50(2), 179-211.

Albright, C., Pruitt, L., Castro, C., Gonzalez, A., Woo, S., \& King, A. (2005). Modifying physical activity in a multiethnic sample of low-income women: One-year results from the IMPACT (Increasing Motivation for Physical ACTivity) project. Annals of Behavioral Medicine, 30(3), 191-200.

Allan, J.D. (1987). Identification of health risks in a young adult population. Journal of Community Health Nursing, 4(4), 223-233.

Anderson, D.A., Shapiro, J.R., \& Lundgren, J.D. (2003). The freshman year of college as a critical period for weigh gain: An initial evaluation. Eating Behaviors, 4(4), 363-367

Anderson, K. (2001). Internet use among college students: An exploratory study. Journal of American College Health, 50(1), 21-26. 
Anderson, L.M., Quinn, T.A., Glanz, K., Ramirez, G., Kahwati, L.C., Johnson, D.B., Buchanan, L.R., Archer, W.R., Chattopadhyay, S., Kalra, G.P., \& Katz, D.L. (2009). The effectiveness of worksite nutrition and physical activity interventions for controlling employee overweight and obesity: a systematic review. Task Force on Community Preventive Services, American Journal of Preventive Medicine, 37(4), 340-357

Anding, J.D., Suminski, R.R., \& Boss, L. (2001). Dietary intake, body mass index, exercise, and alcohol: are college women following the dietary guidelines for Americans? Journal of American College Health, 49(4), 167-171.

Armitage, C. (2009). Is there utility in the transtheoretical model? British Journal of Health Psychology, 14(2). 195-210

Armitage, C., \& Arden, M. (2002). Exploring discontinuity patterns in the transtheoretical model: An application of the theory of planned behavior. British Journal of Health Psychology, 7(1), 89-103.

Armitage, C., Povey, R., \& Arden, M. (2003). Evidence for discontinuity patterns across the stages of change: a role for attitudinal ambivalence. Psychology \& Health, 18(3), 373386.

Armitage, C., Sheeran, P., Conner, M., \& Arden, M. (2004). Stages of Change or Changes of Stage? Predicting Transitions in Transtheoretical Model Stages in Relation to Healthy Food Choice. Journal of Consulting and Clinical Psychology, 72(3), 491-499.

Arnett, J.J. (1997). Young people's conceptions of the transition to adulthood. Youth \& Society, 29(1), 1-23

Arnett, J.J. (1998). Learning to stand alone: the contemporary American transition to adulthood in cultural and historical context. Human Development, 41(5-6), 295-315 
Arnett, J.J. (2000). Emerging adulthood: A theory of development from the late teens through the twenties. American Psychologist, 55(5), 469-480.

Arnett, J.J. (2001). Conceptions of the transition to adulthood from adolescence through midlife. Journal of Adult Development, 8(2), 135-145

Arnett, J.J. (2004). Emerging Adulthood: The winding road from the late teens through the twenties. New York: Oxford University Press

Ball, K., Owen, N., Salmon, J., Bauman, A., \& Gore, C.J. (2001). Associations of physical activity with body weight and fat in men and women. International Journal of Obesity, 25(6), 914-919

Ball, K., Salmon, J., Leslie, E., Owen, N., \& King, A. (2005). Piloting the feasibility and effectiveness of print- and telephone-mediated interventions for promoting the adoption of physical activity in Australian adults. Journal of Science and Medicine in Sport / Sports Medicine Australia, 8(2), 134-142.

Baranowski, T., Anderson, C., Carmack., C. (1998). Mediating variable framework in physical activity interventions. How are we doing? How might we do better? American Journal of Preventive Medicine, 15(4), 266-297

Barnes, P. \& Schoenborn, C. (2003) Physical activity among adults: United States, 2000. Advance Data from Health and Vital Statistics, 333, 1-24.

Bartlett, M.L. (2009). The evaluation of a nutrition education and fitness program with a contest component among college students using the RE-AIM framework. (Unpublished doctoral disseration). West Virginia University, Morgantown, WV 
Bauer, K., Larson, N.I., Nelson, M., Story, M., \& Neumark-Sztainer, D. (2009). Fast food intake among adolescents: Secular and longitudinal trends from 1999-2004. Preventive Medicine, 48(3), 284-28

Beers, T., Hohman, M., Slack, K., Sisneros, D., \& Woodruff, S. (2009). Brief telephone-based interventions for clients with alcohol and other drug abuse problems. The Praeger international collection on addictions, Vol 3: Characteristics and treatment perspectives (pp. 289-304). Santa Barbara, CA US: Praeger/ABC-CLIO.

Bennett, G., \& Galsgow, R. (2009). The delivery of public health interventions via the Internet: Actualizing their potential. Annual Review of Public Health, 30(1), 273-292.

Braunstein, J.B. (2004). Lifestyle activity. Exercise for the masses. Diabetes Forecast, 57(5), 3134

Bray, S.R. \& Born, H.A. (2004). Transition to university and vigorous physical activity: Implications for health and psychological well-being. Journal of American College Health, 52(4), 181-188.

Bridle, C., Riemsma, R., Pattenden, J., Sowden, A., Mather, L., Watt, I., et al. (2005). Systematic review of the effectiveness of health behavior interventions based on the transtheoretical model. Psychology \& Health, 20(3), 283-301.

Brown, C. (2008). The information trail of the 'Freshman $15^{\prime}$ - a systematic review of a health myth within the research and popular literature. Health Information and Libraries Journal, 25(1), 1-12

Brownell, K.D. \& Battle Horgen, K. (2004). Food Fight The Inside Story of the Food Industry, America's Obesity Crisis, and What We Can Do About It. New York, NY: McGraw-Hill Brug, J., Oenema, A., Kroeze, W., \& Raat, H. (2005). The internet and nutrition education: 
challenges and opportunities. European Journal of Clinical Nutrition, 59, S130-S139.

Brunsting, S., \& van den Putte, B. (2006). Web-based computer-tailored feedback on alcohol use: Motivating excessive drinkers to consider their behavior. In Murero, M. \& Rice, R.E. (Eds.), The Internet and health care: Theory, research, and practice (pp. 313-333). Mahwah, NJ: Erlbaum.

Burke, B. L., Arkowitz, H., \& Menchola, M. (2003). The efficacy of motivational interviewing: A meta-analysis of controlled clinical trials. Journal of Consulting and Clinical Psychology, 71(5), 843-861.

Butler, S.M., Black, D.R., Blue, C.L., Gretebeck, R.J. (2004). Change in diet, physical activity, and body weight in female college freshman. American Journal of Health Behavior, $28(1), 24-32$

Carey, K., Scott-Sheldon, L., Carey, M., \& DeMartini, K. (2007). Individual-level interventions to reduce college student drinking: A meta-analytic review. Addictive Behaviors, 32(11), 2469-2494.

Carron, A. V., Hausenblas, H. A., \& Estabrooks, P. A. (2003). The psychology of physical activity. New York: McGraw-Hill.

Caspersen, C.J., Pereira, M.A., \& Curran, K.M. (2000). Changes in physical activity patterns in the U.S., by cross-sectional age and sex. Medicine and Science in Sports and Exercise, 32(9), 1601-1609.

Caspersen, C.J., Powell, K.E., Christensen, G.M. (1985). Physical activity, exercise and physical fitness: definitions and distinctions for health-related research. Public Health Reports, $100(2), 126-131$.

Center for Disease Control and Prevention and Health Promotion. (2010). Prevalence of obesity 
among adults aged 20 years and over: United States, 1997-March 2009. Retrieved from http://www.cdc.gov/nchs/data/nhis/earlyrelease/200909_06.pdf (01/23/10)

Center for Disease Control and Prevention and Health Promotion (2007). Behavioral Risk Factor Surveillance System. Retrieved from http://apps.nccd.cdc.gov/BRFSS/display.asp?cat=FV\&yr=2007\&qkey=4415\&state=US

Center for Disease Control and Prevention U.S. Department of Health and Human Services (2009). Summary health statistics for U.S. adults: National health interview survey, 2008 Provisional Report, 10(242) DHHS Publication No. (PHS) 2010-1570

Chapman, M.C. (2007). Checking out students on Facebook. Chronicle of Higher Education, 53(32), A55. (accessed January 19, 2010).

Chen, C., Storr, C.L., \& Anthony, J.C. (2009). Early-onset drug use and risk for drug dependence problems. Addictive Behaviors, 34(3), 319-322.

Chen, C., Storr, C.L., Tang, G., Huang, S., Hsiao, C.K., Chen, \& Wei, J. (2007). Early alcohol experiences and adolescent mental health: A population-based study in Taiwan. Drug and Alcohol Dependence, 95(3), 209-218

Cleveland, L., Goldman, J., Moshfegh, A. (2005). Contribution of snacks to food and nutrient intakes in the United States [abstract]. The Federation of American Societies for Experimental Biology Journal, 19(4), A88.

Cohen, J. (1988). Statistical power analysis for the behavioral sciences (2nd ed.). Hillsdale, NJ: Lawrence Earlbaum Associates.

Conner, M., \& Sparks, P. (2005). Theory of planned behaviour and health behaviour. In Conner, M. \& Norman, P. (Eds.), Predicting health behaviour (pp. 170-222). London: Open University Press. 
Contento, I.R., Randell, J.S., \& Basch, C.E. (2002). Review and analysis of evaluation measures used in nutrition education intervention research. Journal of Nutrition Education and Behavior, 34(1), 2-25.

Courneya, K. (1995). Understanding readiness for regular physical activity in older individuals: An application of the theory of planned behavior. Health Psychology, 14(1), 80-87.

Courneya, K., Plotnikoff, R., Hotz, S., \& Birkett, N. (2001). Predicting exercise stage transitions over two consecutive 6-month periods: A test of the theory of planned behaviour in a population-based sample. British Journal of Health Psychology, 6(2), 135-150.

Crissey, S.R. (2009). Population Characteristics Educational Attainment in the United States: 2007. Available at: http://www.census.gov/population/www/socdemo/educ-attn.html. Accessed $11 / 30 / 2009$

Cullen, K.W., Koehly, L.M., Anderson, C., Baranowski, T., Prokhorov, A., Basen-Engquist, K., Wetter, D. Hergenroeder, A. (1999). Gender differences in chronic disease risk behaviors through the transition out of high school. American Journal of Preventive Medicine, $17(1), 1-7$

Cunningham, J., Wild, T., Cordingley, J., van Mierlo, T., \& Humphreys, K. (2009). A randomized controlled trial of an internet-based intervention for alcohol abusers. Addiction, 104(12), 2023-2032.

Curioni, C., \& Lourenço, P. (2005). Long-term weight loss after diet and exercise: A systematic review. International Journal of Obesity, 29(10), 1168-1174.

Dansinger, M., Tatsioni, A., Wong, J., Mei, C., \& Balk, E. (2007). Meta-analysis: The effect of dietary counseling for weight loss. Annals of Internal Medicine, 147(1), 41-W7. 
De Bourdeaudhuij, I., Stevens, V., Vandelanotte, C., \& Brug, J. (2007). Evaluation of an interactive computer-tailored nutrition intervention in a real-life setting. Annals of Behavioral Medicine, 33(1), 39-48.

de Castro, J.M. (2004). The time of day of food intake influences overall intake in humans. The Journal of Nutrition, 134(1), 104-111

de Vries, H., \& Brug, J. (1999). Computer-tailored interventions motivating people to adopt health promoting behaviours: Introduction to a new approach. Patient Education and Counseling, 36(2), 99-195.

Del Boca, F. K., Darkes, J., Greenbaum, P. E., \& Goldman, M. S. (2004). Up close and personal: Temporal variability in the drinking of individual college students during their first year. Journal of Consulting and Clinical Psychology, 72(2), 155-164.

Demory-Luce, D., Morales, M., Nicklas, T., Baranowski, T., Zakeri, I., \& Berenson, G. (2004). Changes in food group consumption patterns from childhood to young adulthood: the Bogalusa Heart Study. Journal of the American Dietetic Association, 104(11), 16841691.

Di Noia, J., Contento, I., \& Prochaska, J. (2008). Computer-mediated intervention tailored on transtheoretical model stages and processes of change increases fruit and vegetable consumption among urban African-American adolescents. American Journal of Health Promotion: AJHP, 22(5), 336-341.

Dijkstra, A. (2005). Working mechanisms of computer-tailored health education: Evidence from smoking cessation. Health Education Research, 20(5), 527-539.

Dijkstra, A. \& DeVries, H. (1999) The development of computer-generated tailored interventions. Patient education and counseling, 36(2), 193-203 
Dishman, R.K. and Buckworth, J. (1996). Increasing physical activity: a quantitative synthesis. Medicine and science in sports and exercise, 28(6), 706-719. [Abstract]

Driskell, J.A., Kim, Y., Goebel, K. (2005). Few differences found in the typical eating and physical activity habits of lower-level and upper-level university students. Journal of the American Dietetic Association, 105(5), 798-801

Dugdill, L., Crone, D., \& Murphy, R. (Ed.s) (2009). Physical activity and health promotion: Evidence-based approaches to practice. Oxford, UK: Wiley-Blackwell Publishing, Ltd.

Dworkin, J. (2005). Risk taking as developmentally appropriate experimentation for college students. Journal of Adolescent Research, 20(2), 219-241

Eaton, D.K., Kann, L., Okoro, C.A., \& Collins, J. (2007). Selected health status indicators and behaviors of young adults, United States-2003. American Journal of Health Education, $38(2), 66-75$

Eakin, E.G., Lawler, S.P., Vandelanotte, C., Owen, N. (2007). Telephone interventions for physical activity and dietary behavior change: a systematic review. American Journal of Preventive Medicine, 32(5), 419-434.

Kroeze, W., Werkman, A., Brug J. (2006). Effectiveness of computer-tailored education on physical activity and dietary behaviors. Annals of Behavioral Medicine, 31(3), 205-223.

Engelmore, R.S. \& Feigenbaum, E. (1993). Expert systems and artificial intelligence. In: Engelmore, R.S. (Ed.) Knowledge-Based systems in Japan. Retrieved from: http://www.wtec.org/loyola/kb/toc.htm

Erikson, E.H. (1950). Childhood and society. New York: Norton.

Fahey, T.D., Insel, P.M., \& Roth, W.T. (2003). Fit \& well: Core concepts and labs in physical fitness and wellness $\left(5^{\text {th }}\right.$ Ed.). New York: McGraw-Hill. 
Ferrara, C.M. (2009). The college experience: physical activity, nutrition, and implications for intervention and future research. Journal of Exercise Physiology Online, 12(1), 23-35

Ferrara, C.M., Laurent, C., \&Wilson, T. (2008). The benefits of a weight loss contest in overweight and obese college students. Recreational Sports Journal, 32(1), 45-51

Ferreira, I., Twisk, J.W., van Mechelen, W., Kemper, H.C., \& Stehouwer, C.D. (2005). Development of fatness, fitness, and lifestyle from adolescence to the age of 36 years: determinants of the metabolic syndrome in young adults: the Amsterdam growth and health longitudinal study. Archives of Internal Medicine, 165(1), 42-48.

Flegal, K., Carroll, M., Ogden, C., \& Curtin, L. (2010). Prevalence and trends in obesity among US adults, 1999-2008. JAMA: The Journal of the American Medical Association, 303(3), 235-241.

Fong, G.T., and Hall, P.A. (2003). The importance of time perspective in predicting, understanding, and reducing health risk behaviors among adolescents. In: Romer, D. (Ed.), Reducing adolescent risk: Toward an integrated approach. Sage, Newbury Park, CA.

Fox, C., Barr-Anderson, D., Neumark-Sztainer, D., \& Wall, M. (2010). Physical activity and sports team participation: Associations with academic outcomes in middle school and high school students. Journal of School Health, 80(1), 31-37.

Freemantle, N., Gill, P., Godfrey, C., Long, A., Richards, C., Sheldon, T., et al. (1993). Brief interventions and alcohol use. Quality in Health Care: QHC, 2(4), 267-273.

Goodman, C. \& Ayodola, A. (2006). What is known about the effectiveness of economic instruments to reduce consumption of foods high in saturated fats and other energy-dense 
foods for preventing and treating obesity? Copenhagen: HealthEvidence Network; World Health Organization 2006.

Gordon, C., Juang, L., \& Syed, M. (2007). Internet use and well-being among college students: Beyond frequency of Use. Journal of College Student Development, 48(6), 674-688.

Gordon-Larsen, P., Adair, L.S., Nelson, M.C., \& Popkin, B.M. (2004). Five-year obesity incidence in the transition period between adolescence and adulthood: the National Longitudinal Study of Adolescent Health. American Journal of Clinical Nutrition, 80(3), $569-575$

Graham, M., \& Jones, A. (2002). Freshman 15: Valid theory or harmful myth? Journal of American College Health, 50(4), 171-183.

Greenblatt, J. C. (2000). Patterns of alcohol use among adolescents and associations with emotional and behavioral problems. Department of Health and Human Services, Office of Applied Statistics Working Paper. Available at http//www.samhsa/gov/oas/oas.html

Guida, G. \& Berini, G. (2000). Ingengeria della conoscenza. Milano, Italy: EGEA.

Guthrie, J.F., Lin, B.H., \& Frazao, E. (2002). Role of food prepared away from home in the American diet, 1977-78 vs 1994-96: Changes and consequences. Journal of Nutritional Education and Behavior, 34(3), 140-150.

Haerens, L., Deforche, B., Maes, L., Brug, J., Vandelanotte, C., \& De Bourdeaudhuij, I. (2007). A computer-tailored dietary fat intake intervention for adolescents: results of a randomized controlled trial. Annals of Behavioral Medicine: A Publication of the Society of Behavioral Medicine, 34(3), 253-262.

Hagger, M., \& Chatzisarantis, N. (2009). Integrating the theory of planned behaviour and selfdetermination theory in health behaviour: A meta-analysis. British Journal of Health 
Psychology, 14(2), 275-302.

Hall, P.A. and Fong, G.T. (2003). The effects of a bref tiem perspective intervention for increasing physical activity among young adults. Psychology and Health, 18(6), 685-706

Hargittai, E., \& Hinnant, A. (2008). Digital inequality: Differences in young adults' use of the internet. Communication Research, 35(5), 602-621.

Hargittai, E., \& Shafer, S. (2006). Differences in actual and perceived online skills: The role of gender. Social Science Quarterly, 87(2), 432-448.

Harvey-Berino, J., Pintauro, S., Buzzell, P., DiGuilio, M., Gold, B., Moldovan, C., et al. (2002). Does using the Internet facilitate the maintenance of weight loss? International Journal of Obesity, 26(9), 1254-1260.

Haskell, W.L., Lee, I.M., Pate, R.R., Powell, K.E., Blair, S.N., Franklin, B.A., Macera, C.A., Heath, G.W., Thompson, P.D., \& Bauman, A. (2007). Physical activity and public health: updated recommendation for adults from the American College of Sports Medicine and the American Heart Association. Circulation, 116(9), 1081-1093

Hemmingsson, E. \& Ekelund, U. (2007). Is the association between physical activity and body mass index obesity dependent? International Journal of Obesity, 31(4), 663-668

Hills, A.P. \& Byrne, N.M. (2006). State of the science: a focus on physical activity. Asia Pacific Journal of Clinical Nutrition, 15, Supplement, 40-48

Hingson, R., Heeren, T., Winter, M., \& Wechsler, H. (2005). Magnitude of alcohol-related mortality and morbidity among U.S. college students ages 18-24: Changes from 1998 to 2001. Annual Review of Public Health, 26, 259-279

Hoffman, D.J., Policastro, P., Quick, V., \& Lee, S.K. (2006). Changes in body weight and fat mass of men and women in the first year of college: A study of the "freshman 15". 
Journal of American College Health, 55(1), 41-45

Hogan, D.P. (1978). The variable order of events in the life course. American Sociological Review, 43(4), 573-586

Holm-Denoma, J., Joiner, T., Vohs, K., \& Heatherton, T. (2008). The 'freshman fifteen' (the 'freshman five' actually): Predictors and possible explanations. Health Psychology, 27(1, Suppl), S3-S9.

Hull, H., Morrow, M., Heesch, K., Dinger, M., Han, J., \& Fields, D. (2007). Effect of the summer months on body weight and composition in college women. Journal of Women's Health, 16(10), 1510-1515.

Hutchison, A.J., Breckon, J.D., Johnson, L.H. (2008). Physical activity behavior change interventions based on the transtheoretical model: A systematic review. Health Education \& Behavior, 36(5), 829-845

Hyman, D.J., Pavlik, V.N., Taylor, W.C., Goodrick, G.K., \& Moye, L. (2007). Simultaneous vs sequential counseling for multiple behavior change. Archives of Internal Medicine, $167(11), 1152-1158$

Institute of Alcohol Studies (2007). Alcohol and mental health. Available at: www.ias.org.uk/resources/factsheets/mentalhealth.pdf Accessed 11/21/09

Jabs, J. \& Devine, C.M. (2006). Time scarcity and food choices: An overview. Appetite, 26(2), 196-204

Jenkins, A., Christensen, H., Walker, J., \& Dear, K. (2009). The effectiveness of distance interventions for increasing physical activity: A review. American Journal of Health Promotion, 24(2), 102-117.

Jones, S. \& Madden M. (2002). The Internet goes to college: How students are living in the 
future with today's technology. Available at:

http://www.pewinternet.org/ /media//Files/Reports/2002/PIP_College_Report.pdf.pdf Accessed $11 / 20 / 09$

Jozkowski, K. (2007). Evaluation of a weight loss intervention program among college females. The Health Education Monograph Series, 24(2), 32-37.

Jung, T., \& Heald, G. (2009). The effects of discriminate message interventions on behavioral intentions to engage in physical activities. Journal of American College Health, 57(5), $527-535$.

Kan, E.B., Ramsey, L.T., Brownson, R.C., Heath, G.W., Howze, E.H., Powell, K.E., Stone, E.J., Rajab, M.W., Corso, P., and the Task Force on Community Preventive Services. (2002). The effectiveness of interventions to increase physical activity. A systematic review. American Journal of Preventive Medicine, 22(4S), 73-107

Kant, A.K. \& Graubard, B.J. (2006). Secular trends in patterns of self-reported food consumption of adult Americans: NHANES 1971-1975 to NHANES 1999-2002. American Journal of Clinical Nutrition, 84(5), 1215-1223

Kasparek, D.G., Corwin, S.J., Valois, R.F., Sargent, R.G. \& Morris, R.L. (2008). Selected health behaviors that influence college freshman weight changes. Journal of American College Health, 56(4), 437-444

Keating, X.D. Guan, J. Piñero, José C., \& Bridges, D.M. (2005). A meta-analysis of college students' physical activity behaviors. Journal of American College Health, 54(2), 116125.

Keeling, R.P. (2000). Resiliency and prevention in college health. Journal of American College Health, 49(1), 3-6 
Kelder, S.H., Perry, C.L., Klepp, K.I. \& Lytle, L.L. (1994). Longitudinal tracking of adolescent smoking, physical activity, and food choice behaviors. American Journal of Public Health, 84(7), 1121-1126

Kelly, S., \& Melnyk, B. (2008). Systematic review of multicomponent interventions with overweight middle adolescents: implications for clinical practice and research. Worldviews on Evidence-Based Nursing / Sigma Theta Tau International, Honor Society of Nursing, 5(3), 113-135.

Keough, K., Zimbardo, P., \& Boyd, J. (1999). Who's smoking, drinking, and using drugs? Time perspective as a predictor of substance use. Basic and Applied Social Psychology, 21(2), 149-164.

Kilpatrick, M., Hebert, E., \& Bartholomew, J. (2005). College student' motivation for physical activity: Differentiating men's and women's motives for sport participation and exercise. Journal of American College Health, 54(2), 87- 94

Kim, Y., Pike, J., Adams, H., Cross, D., Doyle, C., \& Foreyt, J. (2010). Telephone intervention promoting weight-related health behaviors. Preventive Medicine, 50(3), 112-117.

Kolodinsky, J., Harvey-Berino, J.R., Berlin, L., Johnson, R.K., \& Reynolds, T.W. (2007). Knowledge of current dietary guidelines and food choice by college students: better eaters have higher knowledge of dietary guidance. Journal of the American Dietetic Association, 107(8), 1409-1413

Kosma, M., Cardinal, B., \& McCubbin, J. (2005). A pilot study of a web-based physical activity motivational program for adults with physical disabilities. Disability \& Rehabilitation, 27(23), 1435-1442.

Kosma, M., Ellis, R., Cardinal, B., Bauer, J., \& McCubbin, J. (2007). The mediating role of 
intention and stages of change in physical activity among adults with physical disabilities: An integrative framework. Journal of Sport \& Exercise Psychology, 29(1), 21-38.

Kreuter, M., Farrell, D., Olevitch, L., \& Brennan, L. (2000). Tailoring health messages: Customizing communication with computer technology. Mahwah, NJ US: Lawrence Erlbaum Associates Publishers.

Kreuter, M., Strecher, V., \& Glassman, B. (1999). One size does not fit all: The case for tailoring print materials. Annals of Behavioral Medicine, 21(4), 276-283.

Kristal, A., Curry, S., Shattuck, A., Feng, Z., \& Li, S. (2000). A randomized trial of a tailored, self-help dietary intervention: The Puget Sound eating patterns study. Preventive Medicine: An International Journal Devoted to Practice and Theory, 31(4), 380-389.

Kristjánsson, Á., Sigfúsdóttir, I., Allegrante, J., \& Helgason, Á. (2009). Adolescent health behavior, contentment in school, and academic achievement. American Journal of Health Behavior, 33(1), 69-79.

Krivak, T. (2008). Facebook 101: Ten things you need to know about Facebook. (cover story). Information Today, 25(3), 1-44.

Kroeze, W., Werkman, A., \& Brug, J. (2006). A systematic review of randomized trials on the effectiveness of computer-tailored education on physical activity and dietary behaviors. Annals of Behavioral Medicine: A Publication of the Society of Behavioral Medicine, $31(3), 205-223$

Kvaavik, E., Andersen, L.F., \& Klepp, K.I. (2005). The stability of soft drinks intake from adolescence to adult age and the association between long-term consumption of soft drinks and lifestyle factors and body weight. Public Health Nutrition, 8(2), 149-157 
Kwak, L., Kremers, S.P., Bergman, P., Ruiz, J.R., Rizzo, N.S., \& Sjöström, M. (2009).

Associations between physical activity, fitness, and academic achievement. The Journal of Pediatrics, 155(6), 914-918.

Kypri, K., Saunders, J., Williams, S., McGee, R., Langley, J., Cashell-Smith, M., et al. (2004). Web-based screening and brief intervention for hazardous drinking: A double-blind randomized controlled trial. Addiction, 99(11), 1410-1417.

LaPerriere, A., Ironson, G., Antoni, M.H., Schneiderman, N., Klimas, N., \& Fletcher, M.A. (1994). Exercise and psychoneuroimmunology. Medicine \& Science in Sports \& Exercise, 26(2), 182-190.

Largo-Wight, E., Todorovich, J.R., O'Hara, B.K (2008). The effectiveness of a point-based physical activity program. The Physical Educator, 65(1), 30-45

Larimer, M. E., \& Cronce, J. M. (2002). Identification, prevention and treatment: A review of individual-focused strategies to reduce problematic alcohol consumption by college students. Journal of Studies on Alcohol, 14, Supplement 148-163

Larson, N.I. (2008). A longitudinal investigation of diets in transition: What influences dietary patterns during the transition from adolescence to young adulthood? (Unpublished doctoral dissertation), University of Minnesota, Twin Cities, MN

Larson, N.I., Neumark-Sztainer, D,, Harnack, L., Wall, M., Story, M., \& Eisenberg, M.E. (2008). Fruit and vegetable intake correlates during the transition to young adult. American Journal of Preventive Medicine, 35(1), 33-37.

Larson, N.I., Neumark-Sztainer, D., Story, M., Wall, M.M., Harnack, L.J., \& Eisenberg, M.E. (2008). Fast food intake: Longitudinal trends during the transition to young adulthood and correlates of intake. Journal of Adolescent Health, 43(1), 79-86 
Lee, D.C., Sui, X., Blair, S.N. (2008). Does physical activity ameliorate the health hazards of obesity? British Journal of Sports Medicine, 43(1), 49-51

Leith, L.M. (1994). Foundations of exercise and mental health. Morgantown, WV: Fitness Information Technology

Levitsky, D.A., Halbmaier, C.A., Mrdjenovic, G. (2004). The freshman weight gain: a model for the study of the epidemic of obesity. International journal of obesity and related metabolic disorders, 28(11), 1435-1442.

Lewis, C.E., Jacobs, D.R. Jr., McCreath, H., Kiefe, C.I., Schreiner, P.J., Smith, D.E., \& Williams, O.D. (2000). Weight gain continues in the 1990s: 10-year trends in weight and overweight from the CARDIA study. American Journal of Epidemiology, 151(12), 11721181

Lien, N., Lytle, L.A., \& Klepp, K. (2001). Stability in consumption of fruit, vegetables, and sugary foods in a cohort from age 14 to age 21. Preventive Medicine, 33, 217-226

Lowery, R., Galuska, D.A., Fulton, J.E., Wechsler, H., Kann, L., \& Collins, J.L. (2000). Physical activity, food choice, and weight management goals and practices among U.S. college students. American Journal of Preventive Medicine, 18(1), 18-27.

Lowry, R., Galuska, D.A., Fulton, J.E., Wechsler,H., Kann, L., \& Collins, J.L. (2000). Physical activity, food choice, and weight management goals and practices among US college students. American Journal of Preventive Medicine,18(1), 18-27.

Lox, C., Martin Ginis, K., \& Petruzzello, S. (2006). The psychology of exercise: Integrating theory and practice (2nd ed.). Scottsdale, AZ: Holcomb Hathaway, Publishers 
Lustria, M., Cortese, J., Noar, S., \& Glueckauf, R. (2009). Computer-tailored health interventions delivered over the web: Review and analysis of key components. Patient Education and Counseling, 74(2), 156-173.

Marcus, B., Emmons, K., Simkin-Silverman, L., Linnan, L., Taylor, E., Bock, B., et al. (1998). Evaluation of motivationally tailored vs. standard self-help physical activity interventions at the workplace. American Journal of Health Promotion: AJHP, 12(4), 246-253.

Marcus, B., Napolitano, M., King, A., Lewis, B., Whiteley, J., Albrecht, A., et al. (2007). Telephone versus print delivery of an individualized motivationally tailored physical activity intervention: Project STRIDE. Health Psychology, 26(4), 401-409.

Marcus, B., Nigg, C., Riebe, D., \& Forsyth, L. (2000). Interactive communication strategies: Implications for population-based physical-activity promotion. American Journal of Preventive Medicine, 19(2), 121-126.

Marcus, B., Owen, N., Forsyth, L., Cavill, N., \& Fridinger, F. (1998). Physical activity interventions using mass media, print media, and information technology. American Journal of Preventive Medicine, 15(4), 362-378.

Marcus, B.H. Williams, D.M., Dubbert, P.M., Sallis, J.F., King, A.C., Yancey, A.K., Franklin, B.A., Buchner, D., Daniels, S.R., \& Claytor, R.P. (2006). Physical activity intervention studies. What we know and what we need to know. A scientific statement from the american heart association council on nutrition, physical activity, and metabolism (Subcommittee on physical activity); Council on cardiovascular disease in the young; and the interdisciplinary working group on quality of care and outcomes research.

Circulation; 114 (24), 2739-2752

Marcus, B.H., Dubbert, P.M., Forsyth, L.H., McKenzie, T.L., Stone, E.J., Dunn, A.L., Blair, S.N. 
(2000). Physical activity behavior change: issues in adoption and maintenance. Health Psychology, 19(1S), 32-41

Marmonier, C., Chapelot, D., Fantino, M., \& Louis-Sylvestre, J. (2002). Snacks consumed in a nonhungry state have poor satiating efficiency: influence of snack composition on substrate utilization and hunger. The American Journal of Clinical Nutrition, 76(3), 518528.

Mata, J., Silva, M.N., Vieira, P.N., Carraça E.V., Andrade, A.M., Coutinho, S.C., Sardinha, L.B., \& Teixeira, P.J. (2009). Motivational “Spill-Over” during weight control: Increased selfdetermination and exercise intrinsic motivation predict eating self-regulation. Health Psychology, 28(6), 709-716

Matvienko, O., Lewis, D.S., \& Schafer, E. (2001). A college nutrition science course as an intervention to prevent weight gain in female college freshmen. Journal of Nutrition Education and Behavior, 33, 95-101.

McCracken, M., Jiles, R., \& Blanck, H.M. (2007). Health behaviors of the young adult U.S. population: Behavioral Risk Factor Surveillance System, 2003. Preventing Chronic Disease: Public Health Research, Practice and Policy, 4(2). Available from: http://www.cdc.gov/pcd/issues/2007/apr/06_0090.htm Accessed 11/7/09.

McTigue, K.M., Garrett, J.M., \& Popkin, B.M. (2002). The natural history of the development of obesity in a cohort of young U.S. adults between 1981 and 1998. Annals of Internal Medicine, 136(12), 857-864

Medalie, J. (1981). The college years as a mini-life cycle: developmental tasks and adaptive options. College Health, 30, 75-79.

Mellen, K.R. (2007). Health behaviors of college students: Sample comparisons, physical 
activity associations, and a physical activity intervention. (Unpublished doctoral disseration), The University of Iowa, Iowa City, IA

Michie,S., Abraham, C., Whittington, C., McAteer, J. (2009). Effective techniques in healthy eating and physical activity interventions: A meta-regression. Health Psychology, 28(6), 690-701.

Mihalopoulos, N.L., Auinger, P., \& Klein, J.D. (2008). The Freshman 15: is it real? Journal of American College Health, 56(5), 531-533.

Miles, L. (2007). Physical activity and health. Nutrition Bulletin, 32(4), 314 - 363.

Miller, K.H., Ogletree, R.J., \& Welshimer, K. (2002). Impact of activity behaviors on physical activity identity and self-efficacy. American Journal of Health Behavior, 26(5), 323-330.

Miller, W., Koceja, D., \& Hamilton, E. (1997). A meta-analysis of the past 25 years of weight loss research using diet, exercise or diet plus exercise intervention. International Journal of Obesity and Related Metabolic Disorders: Journal of the International Association for the Study of Obesity, 21(10), 941-947.

Mokdad, A.H., Ford, E.S., Bowman, B.A., Dietz, W.H., Vinicor, F., Bales, V.S., Marks, J.S. et al. (2003). Prevalence of obesity, diabetes, and obesity-related health risk factors, 2001. JAMA: Journal of the American Medical Association, 289(1), 76-79.

Mokdad, A.H., Marks, J.S., Stroup, D.F., \& Gerberding, J.L. (2004). Actual causes of death in the United Stated, 2000. JAMA: The Journal of the American Medical Association, 291(10), 1238-1245.

Mokdad, A.H., Serdula, M.K., Dietz, W.H., Bowan, B.A., Marks, J.A., \& Koplan, J.P. (1999). The spread of the obesity epidemic in the United States, 1991-1998. JAMA: The Journal of the American Medical Association, 282(16), 1519-1522. 
Moore, M. J., Soderquist, J., \& Werch, C. (2005). Feasibility and efficacy of a binge drinking prevention intervention for college students delivered via the Internet versus postal mail. Journal of American College Health, 54(1), 38-44.

Morgan, P.M. (1985). Athletes and non-athletes in the middle years of life. In: McPherson, B.D. (Ed.), Sport and Aging. Champaign, IL: Human Kinetics.

Morgan, P.M. (2001). Prescription of physical activity: A paradigm shift. Quest, 53(3), 366-382.

Napolitano M \& Marcus B. (2002). Targeting and tailoring physical activity information using print and information technologies. Exercise and Sport Sciences Reviews, 30(3), 122-128.

National Institute on Alcohol Abuse and Alcoholism of the National Institute of Health (1996). Alcohol and stress. Alcohol Alert, 32. Available at: http://pubs.niaaa.nih.gov/publications/aa32.htm Accessed 11/22/09

National Telecommunications and Information Administration. (2004). A nation online: Entering the broadband age. Washington, DC: U.S. Department of Commerce.

Neighmond, P. (2010). Obesity Rates Level Off, But Health Concerns Remain. Available at: http://www.wbur.org/npr/122536128. Access 01/30/2010

Nelson, M., Larson, N., Barr-Anderson, D., Neumark-Srtainer, D., \& Story, M. (2009). Disparities in dietary intake, meal patterning, and home food environments among young adult nonstudents and 2- and 4-year college students. American Journal of Public Health, $99(7), 1216-1219$.

Nelson, M., Neumark-Stzainer, D., Hannan, P., Sirard, J., \& Story, M. (2006). Longitudinal and secular trends in physical activity and sedentary behavior during adolescence. Pediatrics, 118(6), e1627-e1634.

Nelson, M., Story, M., Larson, N., Neumark-Sztainer, D., \& Lytle, L. (2008). Emerging 
adulthood and college-aged youth: An overlooked age for weight-related behavior change. Obesity, 16(10), 2205-2211.

Nelson, M.C., Lust, K., Story, M., \& Ehlinger, E. (2009). Alcohol use, eating patterns, and weight behaviors in a university population. American Journal of Health Behaviors, $33(3), 227-237$.

Nelson, M.C., Neumark-Stzainer, D., Hannan, P., Sirard, J., \& Story, M. (2006). Longitudinal and secular trends in physical activity and sedentary behavior during adolescence. Pediatrics, 118(6), e1627-e1634.

Nelson, M.C., Story, M., Larson, N.I., Neumark-Sztainer, D., \& Lytle, L.A. (2008). Emerging adulthood and college-aged youth: an overlooked age for weight-related behavior change. Obesity, 16(10), 2205-2011.

Neumark-Sztainer, D., Wall, M., Eisenberg, M.E., Story, M., \& Hannan, P.J. (2006b). Overweight status and weight control behaviors in adolescents: longitudinal and secular trends from 1999 to 2004. American Journal of Preventive Medicine, 43(1), 52-59.

Neumark-Sztainer, D., Wall, M., Guo, J., Story, M., Haines, J., Eisenberg, M. (2006a). Obesity, disordered eating, and eating disorders in a longitudinal study of adolescents: how do dieters fare 5 years later? Journal of American Dietetic Association, 106(4), 559-568.

Neville, L., Milat, A., \& O'Hara, B. (2009). Computer-tailored weight reduction interventions targeting adults: a narrative systematic review. Health Promotion Journal of Australia: Official Journal of Australian Association of Health Promotion Professionals, 20(1), 4857.

Neville, L., O’Hara, B., \& Milat, A. (2009). Computer-tailored dietary behaviour change interventions: A systematic review. Health Education Research, 24(4), 699-720. 
Neville, L., O'Hara, B., \& Milat, A. (2009). Computer-tailored physical activity behavior change interventions targeting adults: A systematic review. The International Journal of Behavioral Nutrition and Physical Activity, 6, 30-42.

Newton, F., Kim, E., \& Newton, D. (2006). A program to establish healthy lifestyle behaviors with freshmen Students. NASPA Journal, 43(3), 497-517.

Nielsen, S.J., Barry, B.S., Popkin, M. (2004). Changes in beverage intake between 1977 and 2001. American Journal of Preventive Medicine, 27(3), 205-210.

Niemeier, H.M., Raynor, H.A., Lloyd-Richardson, E.E., Rogers, M.L., \& Wing, R.R. (2006). Fast food consumption and breakfast skipping: predictors of weight gain from adolescence to adulthood in a nationally representative sample. Journal of Adolescent Health, 39(6), 842-849.

Nigg, C. (2003). Technology's influence on physical activity and exercise science: The present and the future. Psychology of Sport and Exercise, 4(1), 57-65.

Nigg, C. R., Riebe, D., Rossi, J. S., Velicer, W. F., \& Prochaska, J. 0. (June, 1999). Individualized expert system interventions for adopting and maintaining physical activity. Presentation at ACSM Special Event: Demonstrations of New Information Technology to Promote Physical Activity. Seattle, WA.

Nii, H.P. (1993). Expert systems building tools: Definition. . In: Engelmore, R.S. (Ed.) Knowledge-Based Systems in Japan. Retrieved from: http://www.wtec.org/loyola/kb/toc.htm

Odell, P., Korgen, K., Schumacher, P., \& Delucchi, M. (2000). Internet use among female and male college students. CyberPsychology \& Behavior, 3(5), 855-862. 
Odgen, C.L., Carroll, M.D., Curtin, L.R., McDowell, M.A., Tabak, C.J., \& Flegal, K.M. (2006). Prevalence of overweight and obesity in the United States, 1999-2004. JAMA: The Journal of the American Medical Association, 295(13), 1549-1555.

Ogden, C., Carroll, M., Curtin, L., Lamb, M., \& Flegal, K. (2010). Prevalence of high body mass index in US children and adolescents, 2007-2008. JAMA: The Journal of the American Medical Association, 303(3), 242-249.

Paeratakul, S., Ferdinand, D., Champagne, C., Ryan, D., \& Bray, G. (2003). Fast-food consumption among US adults and children: dietary and nutrient intake profile. Journal of the American Dietetic Association, 103(10), 1332-1338.

Paeratakul, S., Ferdinand, D.P., Champagne, C.M., Ryan, D.H., \& Bray, G.A. (2003). Fast-food consumption among US adults and children: dietary and nutrient intake profile. Journal of the American Dietetic Association, 103(10), 1332-1338.

Park, B., Lee, M., Hong, J., Kim, E., Kim, D., Kim, K., et al. (2009). The stages of physical activity and exercise behavior: An integrated approach to the theory of planned behavior. Asia-Pacific Journal of Public Health, 21(1), 71-83.

Park, M.J., Mulye, T.P., Adams, S.H., Brindis, C.D., Irwin, C.E. (2006). The health status of young adults in United States. Journal of Adolescent Health, 39(3), 305-317.

Pate, R.R., Heath, G.W., Dowda, M., \& Trost, S.G. (1996). Association between physical activity and other health behaviors in a representative sample of US adolescents. American Journal of Public Heath, 86(11), 1577-1581.

Pliner, P. \& Saunders, T. (2008). Vulnerability to freshman weight gain as a function of dietary restraint and residence. Physiology \& behavior, 93(1-2), 76-82. 
Plotnikoff, R.C., McCargar, L.J., Wilson, P.M., \& Loucaides, C.A. (2005). Efficacy of an e-mail intervention for the promotion of physical activity and nutrition behavior in the workplace context. American Journal of Health Promotion, 19(6), 422-429.

Pomerleau, J., Lock, K., Knai, C., \& McKee, M. (2005). Interventions designed to increase adult fruit and vegetable intake can be effective: A systematic review of the literature. Journal of Nutrition, 135(10), 2486-2495.

Powell, J., Darvell, M., \& Gray, J. (2003). The doctor, the patient and the world-wide web: how the internet is changing healthcare. Journal of the Royal Society of Medicine, 96(2), 7476.

Pratt, M. (1999). Benefits of lifestyle activity vs. structured exercise. JAMA: The Journal of the American Medical Association, 281(4), 375-376.

Prochaska, J., Redding, C., Harlow, L., Rossi, J., \& Velicer , W.F. (1994a). The transtheoretical model of change and HIV prevention: A review. Health Education Quarterly, 21(4), 471486.

Prochaska, J.O. \& DiClemente, C.C. (1982). Trans-theoretical therapy - toward a more integrative model of change. Psychotherapy: Theory, Research and Practice, 19(3), 276288.

Prochaska, J.O. \& DiClemente, C.C. (1983). Stages and processes of self-change of smoking: toward an integrative model of change. Journal of Consulting and Clinical Psychology, $51(3), 390-395$.

Prochaska, J.O. \& Velicer, W.F. (1997). The transtheoretical model of health behavior change. American Journal of Health Promotion, 12(1), 38-48. 
Prochaska, J.O., Norcross, J.C., \& DiClemente, C.C. (2002). Changing for good: A revolutionary six-stage program for overcoming bad habits and moving your life positively forward. New York, NY: HarperCollins Publisher, Inc.

Pullman, A.W., Masters, R.C., Zalot, L.C., Carde, L.E., Saraiva, M.M., Dam, Y., Randall Simpson, J.A., \& Duncan, A.M. (2009). Effect of the transition from high school to university on anthropometric and lifestyle variables in males. Applied Nutrition Physiology and Metabolism, 34(2), 1-10.

Raacke, J., \& Bonds-Raacke, J. (2008). MySpace and Facebook: Applying the uses and gratifications theory to exploring friend-networking sites. CyberPsychology \& Behavior, 11(2), 169-174.

Racette SB, Deusinger SS, Strube MJ, Highstein GR, Deusinger RH: (2008) Changes in weight and health behaviors from freshman through senior year of college. Journal of Nutrition Education and Behavior, 40(1), 39-42.

Racette, R.B., Deusinger, S.S., Highstein, G.R., Strube, M.J., \& Deusinger, R.H. (2005). Weight changes, exercise, and dietary patterns during freshman and sophomore years of college. Journal of American College Health, 53(6), 245-251.

Ravert, R.D. (2009). "You're only young once": Things college students report doing now before it is too late. Journal of Adolescent Research, 24(3), 376-396.

Richardson, S. \& Barrow, L. (2000). Academic effects of alcohol consumption. Available at : www.facstaff.bucknell.edu/awolaver/alcohol/academic.doc. Accessed 11/22/09

Rozin, P., Bauer, R., \& Catanese, D. (2003). Food and life, pleasure and worry, among American college students: gender differences and regional similarities. Journal of Personality and Social Psychology, 85(1), 132-141 
Rubin, A., Migneault, J., Marks, L., Goldstein, E., Ludena, K., \& Friedman, R. (2006).

Automated telephone screening for problem drinking. Journal of Studies on Alcohol, $67(3), 454-457$.

Ryan, P., \& Lauver, D. (2002). The Efficacy of Tailored Interventions. Journal of Nursing Scholarship, 34(4), 331-337.

Sallis, J.F., Prochaska, J.J., Taylor, W.C. (2000). A Review of correlates of physical activity of children and adolescents. Medicine and Science in Sport and Exercise, 32(5), 963-975.

Sanchez, A., Norman, G.J., Sallis, J.F., Calfas, K.J., Cella, J., \& Patrick, K. (2007). Patterns and correlates of physical activity and nutrition behaviors in adolescents. American Journal of Preventive Medicine, 32(2), 124-130.

Schröder, H., Morales-Molina, J.A., Bermejo, S., Barral, D., Mándoli Soler, E., Grau, M., Guxens, M., de Jaime Gil, E., Álvarez, Domínguez, M. Marrugat, J. (2007). Relationship of abdominal obesity with alcohol consumption at population scale. European Journal of Nutrition, 46(7), 369-376.

Schulenberg, J.E., Zarrett, N.R. (2006). Mental health during emerging adulthood: Continuity and discontinuity in courses, causes, and functions. In: Arnett, J.J. \& Tanner, J.L. (Ed.s). Emerging adults in America: Coming of age in the 21st century. Washington, DC, US: American Psychological Association, 135-172.

Schulte, M.T., Ramo, D., \& Brown, S.A. (2009) Gender differences in factors influencing alcohol use and drinking progression among adolescents. Clinical Psychology Review, 29(6), 535-547.

Schwartz, S.J., Côté, J.E., \& Arnett, J.J. (2005). Process identity and agency in emerging adulthood: Two developmental routes in the individualization. Youth Society, 37(4), 201- 
229.

Scott-Sheldon, L., DeMartini, K. Carey, K., \& Carey, M., (2009). Alcohol interventions for college students improves antecedents of behavioral change: results from a meta-analysis of 34 randomized controlled trials. Journal of Social \& Clinical Psychology, 28(7), 799823.

Sebastian, R.S., Cleveland, L.E., \& Goldman, J.D. (2008). Effect of snacking frequency on adolescents' dietary intakes and meeting national recommendations. Journal of Adolescent Health, 42(5), 503-511.

Singleton, R.A. \& Wolfson, A.R. (2009). Alcohol consumption, sleep, and academic performance among college students. Journal of Studies on Alcohol and Drugs, 70(3), 355-363 [Abstract].

Skinner, C., Campbell, M., Rimer, B., Curry, S., \& Prochaska, J. (1999). How effective is tailored print communication? Annals of Behavioral Medicine, 21(4), 290-298.

Skinner, H., Morrison, M., Bercovitz, K., Haans, D., Jennings, M., Magdenko, L., et al. (1997). Using the Internet to engage youth in health promotion. Promotion \& Education, 4(4), 23-25.

Sneed, J., \& Holt, C. (1991). Many factors influence college students' eating patterns. Journal of The American Dietetic Association, 91(11), 1380

Snyder, T.D., Dillow, S.A., \& Hoffman, C.M. (2009). Digest of Education Statistics 2008. Washington, DC: U.S. Government Printing Office.

Soh, N.L., Walter, G., Baur, L., \& Collins, C. (2009). Nutrition, mood and behaviour: A review. Acta Neuropsychiatrica, 21(5), 214-227.

Sparling, P.B. \& Snow, T.K. (2002). Physical activity patterns in recent college alumni. 
Research Quarterly for Exercise \& Sport, 73(2), 200-205

Spittaels, H., De Bourdeaudhuij, I., Brug, J., \& Vandelanotte, C. (2007). Effectiveness of an online computer-tailored physical activity intervention in a real-life setting. Health Education Research, 22(3), 385-396.

Stevens, T., To, Y., Stevenson, S., \& Lochbaum, M. (2008). The importance of physical activity in the prediction of academic achievement. Journal of Sport Behavior, 31(4), 368-388.

Storer, J. H., Cychosz, C. M., \& Anderson, D. F. (1997). Wellness behaviors, social identities, and health promotion. American Journal of Health Behavior, 21(4), 260-268.

Strathman, A., Gleicher, F., Boninger, D., \& Edwards, C. (1994). The consideration of future consequences: Weighing immediate and distant outcomes of behavior. Journal of Personality and Social Psychology, 66(4), 742-752.

Strecher, V., Shiffman, S., \& West, R. (2005). Randomized controlled trial of a web-based computer-tailored smoking cessation program as a supplement to nicotine patch therapy. Addiction, 100(5), 682-688.

Subrahmanyam, K., \& Lin, G. (2007). Adolescents on the Net: Internet use and well-being. Adolescence, 42(168), 659-677.

T., G. (2007). Facebook application helps students choose a college. University Business, 10(11), 18.

Thayer, S., \& Ray, S. (2006). Online communication preferences across age, gender, and duration of internet use. CyberPsychology \& Behavior, 9(4), 432-440.

Toll, B., Sobell, L., D'Arienzo, J., Sobell, M., Eickleberry-Goldsmith, L., \& Toll, H. (2003). What do Internet-based alcohol treatment websites offer? CyberPsychology \& Behavior, 6(6), 581-584. 
Trockel, M., Barnes, M., \& Egget, D. (2000). Health-related variables and academic performance among first-year college students: Implications for sleep and other behaviors. Journal of American College Health, 49(3), 125-131.

U.S. Department of Health and Human Services (1996). The physical activity and health: a report of the surgeon general. Atlanta, GA: USDHHS, Center for Disease Control and Prevention, National Centre for Chronic Disease Prevention and Health Promotion.

U.S. Department of Health and Human Services (2000). Healthy people 2010: Understanding and improving health (2nd ed). Washington, DC: U.S. Government Printing Office.

U.S. Department of Health and Human Services (2008). 2008 Physical activity guidelines for Americans. Washington, DC: U.S. Government Printing Office. Available at: http://www.health.gov/PAGuidelines/pdf/paguide.pdf. Accessed 04/15/09.

U.S. Department of Health and Human Services and U.S. Department of Agriculture (2005). Dietary guidelines for Americans, 2005. (6th Ed), Washington, DC: U.S. Government Printing Office, January 2005. Available at: http://www.health.gov/DietaryGuidelines/Accessed 11/21/09.

U.S. Department of Health and Human Services. Substance Abuse \& Mental Health Service Administration (2008). Results from the 2008 National Survey on Drug Use and Health: National Findings. Available at: http://www.oas.samhsa.gov/NSDUH/2k8NSDUH/tabs/TOC.htm Accessed 11/21/09.

Valkenburg, P., Peter, J., \& Schouten, A. (2006). Friend networking sites and their relationship to adolescents' well-being and social self-esteem. CyberPsychology \& Behavior, 9(5), 584-590.

Van den Berg, M., Vliet Vlieland, T., \& Schoones, J. (2007). Internet-based physical activity 
interventions: A systematic review of the literature. Journal of Medical Internet Research, 9(3), e26.

Vandelanotte, C., De Bourdeaudhuij, I., \& Brug, J. (2004). Acceptability and feasibility of an interactive computer-tailored fat intake intervention in Belgium. Health Promotion International, 19(4), 463-470.

Vandelanotte, C., De Bourdeaudhuij, I., \& Brug, J. (2007). Two-year follow-up of sequential and simultaneous interactive computer-tailored interventions for increasing physical activity and decreasing fat intake. Annals of Behavioral Medicine, 33(2), 213-219.

Vandelanotte, C., De Bourdeaudhuij, I., Sallis, J., Spittaels, H., \& Brug, J. (2005). Efficacy of sequential or simultaneous interactive computer-tailored interventions for increasing physical activity and decreasing fat intake. Annals of Behavioral Medicine, 29(2), 138146.

Vandelanotte, C., Spathonis, K., Eakin, E., \& Owen, N. (2007). Website-delivered physical activity interventions: A review of the literature. American Journal of Preventive Medicine, 33(1), 54-64.

Velicer, W., Prochaska, J., Fava, J., Norman, G., \& Redding, C. (1998). Smoking cessation and stress management: Applications of the transtheoretical model of behavior change. Homeostasis in Health and Disease, 38(5-6), 216-233.

Von Ah, D., Ebert, S., Ngamvitroj, A., Park, N., \& Kang, D. (2004). Predictors of health behaviours in college students. Journal Of Advanced Nursing, 48(5), 463-474.

Wang, Y. \& Lobstein, T. (2006). Worldwide trends in childhood overweight and obesity. International Journal of Pediatric Obesity, 1(1), 11-25. 
Wang, Y., Beydoun, M.A., Liang, L., Caballero, B., \& Kumanyika, S.K. (2008). Will Americans become overweight or obese? Estimating the progression and cost of the US obesity epidemic. Obesity, 16(10), 2323-2330.

Wang, Y., Monteiro, C., \& Popkin, B.M. (2002). Trends of obesity and underweight in older children and adolescents in the United States, Brazil, China, and Russia. American Journal of Clinical Nutrition, 75(6), 971-977.

Wechsler, H., Kelley, K., Weitzman, E., San Giovanni, J., \& Seibring, M. (2000). What colleges are doing about student binge drinking. Journal of American College Health, 48(5), 219226.

Wechsler, H., Seibring, M., Liu, I. C., \& Ahl, M. (2004). Colleges respond to student binge drinking: Reducing student demand or limiting access. Journal of American College Health, 52, 159-168.

Weiser, E. (2000). Gender differences in Internet use patterns and Internet application preferences: A two-sample comparison. CyberPsychology \& Behavior, 3(2), 167-177. Wetter, A.C., Goldberg, J.P., King, A.C., Sigman-Grant, M., Baer, R., Crayton, E., Devine. C., Drewnowski, A., Dunn, A., Johnson, G., Pronk, N., Saelens, B., Snyder, D., Novelli, P., Walsh, K., \& Warland, R. (2001). How and why do individuals make food and physical activity choices? Nutrition Reviews, 59(3), S11-S20

World Health Organization (2007). WHO Global InfoBase online [online database]. Geneva, Switzerland: World Health Organization, 2007 (noncommunicable diseases, comparable estimates; http://www.who.int/ncd_surveillance/infobase/web/InfoBaseCommon, accessed 8 April 2007). 
Wilcox, S., Parra-Medina, D., Thompson-Robinson, M., \& Will, J. (2001). Nutrition and Physical Activity Interventions to Reduce Cardiovascular Disease Risk in Health. Nutrition Reviews, 59(7), 197-214.

World Health Organization (1948). Preamble to the Constitution of the World Health Organization as adopted by the International Health Conference, New York, 19-22 June, 1946; signed on 22 July 1946 by the representatives of 61 States (Official Records of the World Health Organization, no. 2, p. 100) and entered into force on 7 April 1948.

Available at: http://www.who.int/about/definition/en/print.html. Accessed 11/21/09.

World Health Organization (2002). The Physical inactivity a leading cause of disease and disability, warns WHO. Geneva, Switzerland: World Health Organization Media Release. Available at: http://www.who.int/mediacentre/releases/release23/en/print.html/. Accessed November 21, 2009.

World Health Organization (2003). Obesity and overweight fact sheet. Geneva, Switzerland (http://www.who.int/hpr/NPH/docs/gs_obesity.pdf, accessed January 2006).

Wu, T., Gao, X., Chen, M., van Dam, R.M. (2009). Long-term effectiveness of diet-plus-exercise interventions vs. diet-only interventions for weight loss: a meta-analysis. Obesity Review, $10,313-23$.

Zick, C.D., Smith, K.R., Brown, B.B., Fan, J.X., \& Kowaleski-Jones, L. (2007). Physical activity during the transition from adolescence to adulthood. Journal of Physical Activity \& Health, 4(2), 125-137.

Zimbardo, P., \& Boyd, J. (1999). Putting time in perspective: A valid, reliable individualdifferences metric. Journal of Personality and Social Psychology, 77(6), 1271-1288.

Zimmerman, C.G. (2002). Health promotion in higher education. In: Turner H.S. \& Hurley, J.L. 
(Eds). The history and practice of college health. Lexington, KY: The University Press of Kentucky; 311-327 
APPENDIX B: Distinction between the three administrations

\begin{tabular}{|c|c|c|c|c|c|}
\hline Administration & Section Included & $\begin{array}{c}\# \\
\text { questions } \\
\text { per } \\
\text { section }\end{array}$ & $\begin{array}{l}\text { Total \# of } \\
\text { questions }\end{array}$ & $\begin{array}{l}\text { \# fuzzy } \\
\text { questions }\end{array}$ & $\begin{array}{c}\text { Total \# } \\
\text { fuzzy } \\
\text { questions }\end{array}$ \\
\hline \multirow{7}{*}{$\begin{array}{c}\text { First } \\
\text { Administration }\end{array}$} & Introduction & 3 & \multirow{7}{*}{48} & 0 & \multirow{7}{*}{57} \\
\hline & PA Behavior & $12(7)$ & & 3 & \\
\hline & Eating Behavior & $14(6)$ & & 3 & \\
\hline & Drinking Behavior & $12(5)$ & & 3 & \\
\hline & $\begin{array}{l}\text { Other Program } \\
\text { Participation }\end{array}$ & 1 & & 0 & \\
\hline & Full Time Status & 1 & & 0 & \\
\hline & Demographics & 5 & & 0 & \\
\hline \multirow{9}{*}{$\begin{array}{c}\text { Second } \\
\text { Administration }\end{array}$} & Introduction & 3 & \multirow{9}{*}{54} & 0 & \multirow{9}{*}{63} \\
\hline & PA Behavior & $12(7)$ & & 3 & \\
\hline & Eating Behavior & $14(6)$ & & 3 & \\
\hline & Drinking Behavior & $12(5)$ & & 3 & \\
\hline & Comparison HS & 3 & & 0 & \\
\hline & Legacy/Adjustment & 2 & & 0 & \\
\hline & Fall Results & 1 & & 0 & \\
\hline & Full Time Status & 1 & & 0 & \\
\hline & Demographics & 6 & & 0 & \\
\hline \multirow{11}{*}{$\begin{array}{c}\text { Third } \\
\text { Administration }\end{array}$} & Introduction & 3 & \multirow{11}{*}{59} & 0 & \multirow{11}{*}{68} \\
\hline & PA Behavior & $12(7)$ & & 3 & \\
\hline & Eating Behavior & $14(6)$ & & 3 & \\
\hline & Drinking Behavior & $12(5)$ & & 3 & \\
\hline & Legacy/Adjustment & 2 & & 0 & \\
\hline & $\begin{array}{l}\text { Midterm Spring } \\
\text { Results }\end{array}$ & 1 & & 0 & \\
\hline & Plans for the Future & 2 & & 0 & \\
\hline & $\begin{array}{l}\text { Other Program } \\
\text { Participation }\end{array}$ & 1 & & 0 & \\
\hline & Facebook & 4 & & 0 & \\
\hline & Evaluation Close & 5 & & 0 & \\
\hline & Evaluation Open & 3 & & 0 & \\
\hline
\end{tabular}




\section{APPENDIX C: Informed Consent}

A tailored wellness intervention for first year college students using internet automatic self generated feedback

Principal Investigator: Dr.Zizzi, Sam

Department: WVU CPASS - Sport and Exercise Psychology

Co-Investigator: Quartiroli, Alessandro

Contact Persons

In the event you experience any side effects related to this research, you should contact Dr. Sam Zizzi at 304.293.0874. If you have any questions, concerns, or complaints about this research, you can contact Alessandro Quartiroli (\#\#\#) \#\#\#-\#\#\#\#.

For information regarding your rights as a research subject, to discuss problems, concerns, or suggestions related to the research, to obtain information or offer input about the research, contact the Office of Research Compliance at (\#\#\#) \#\#\#-\#\#\#.

Purpose and Procedures of the Study

The study aims to understand how the use of an internet-based system can help students to enhance their wellness, facilitate their transitional moment from high school to college, and increase the retention level within WVU. Between 300 and 600 first year students are expected to be enrolled in the study.

You will be asked to fill out a questionnaire regarding your health behaviors four times during the semester. Each time will take approximately 10 to 15 minutes.

You will be randomly assigned (have an equal chance, like flipping a coin) to one of three different groups, but all of you will receive a personalized feedback related to your health and wellness habits at the end of the last administration of the questionnaire. The feedback could also help you to realize potential difficulties related to alcohol, nutrition, and physical activity. If you will feel that you have some drinking difficulties, you can contact the Student Assistent Program at (304) 293-4477. If you want to have better information about your physical activity habits you can reach some informative and practical support at the Student Recreational Center. If you feel you desire to obtain more informatin about nutrition, you can schedule an appointment at Student Health to be referred to a specialist, at (\#\#\#) \#\#\#-\#\#\#\#.

You also will be asked to voluntarily participate in a focus group at the end of the semester, which will require around 60 minutes.

Participation

You are free to withdraw your consent to participate in this study at any time. Your participation is required for course credit, but it is possible for you to log in and not answer all questions and still get course credit. To stop answering questions, simply close your browser. However, if you chose to answer the questions, the system will be able to develop a better feedback focused on how to help you to improve and/or maintain your wellness and health. Your MIX ID will be used 
only to track your answers along the four administrations during the semester, if you chose to do so, and to assure that you obtain your class credit. The MIX ID will not be linked with your answer during the data analysis phase of the research, so you will not be identified and/or associated with your answers. If you elect to request that your results will not be included in the final analysis for the research, please contact Alessandro.Quartiroli@mail.wvu.edu, Confidentiality

Any information about you that is obtained as a result of your participation in this research will be kept as confidential as legally possible. During the focus groups, total confidentiality of the shared information cannot be guaranteed.

If you will want to receive the entire documentation of the informed consent, you can request to obtain it by a formal paper method, contacting Alessandro Quartiroli at Alessandro.Quartiroli@mail.wvu.edu,

I willingly consent to participate in this research.

By clicking here you attest that you are at least 18 years of age. WVU IRB Approval of this study is on file. 
APPENDIX D: The three versions of the Survey

\section{Administration 1}

Please answer the questions on this page and the next page. Then click at the bottom of the next page to see feedback.

Enter your 700 ID number:

1. Please write your MIX ID (e.g. Andrew Lopez --> alopez)

(Type just a period in the text box if you choose not to answer.)

2. In general, how would you define your lifestyle? Would you say

Very healthy

Somewhat Healthy

Somewhat unhealthy

Very unhealthy

I choose not to answer

3. Your gender:

Male

Female

I choose not to answer

Moderate physical activities are those, like walking, light bicycling, yoga, or Pilates that cause small increases in breathing or heart rate.

4. In a usual week how many days do you do 30 or more minutes of moderate activity (only count bouts of at least 10 minutes)?

Days 0 - 7

I choose not to answer

Vigorous physical activities cause large increases in breathing or heart rate, like running, lifting weights, aerobics, or Zumba.

5. Again, in a usual week, how many days do you do 20 or more minutes of vigorous activity (only count bouts of at least 10 minutes)?

Days 0 - 7

I choose not to answer

6. On how many of the past 7 days did you do stretching exercises, such as toe touching, knee bending, or leg stretching?

Days 0 - 7

I choose not to answer 
7. On how many of the past 7 days did you do exercises to strengthen or tone your muscles, such as push-ups, sit-ups, or weight lifting?

Days 0 - 7

I choose not to answer

8. Where do you normally like to exercise?

Indoor

Outdoor

No Exercise

I choose not to answer

"Regular Exercise" includes activities (such as power walking, jogging, swimming, aerobic dancing, biking, rowing, weight lifting, sports, stretching, etc.) 3 times or more per week for 20 minutes or longer each time. Activities that are primarily sedentary, such as bowling or playing golf with a golf cart, are NOT considered exercise.

9. Which of these statements most closely reflects you in your exercise behavior?

I currently do not engage in exercise and I am not thinking about starting within the next 6 months.

I currently do not exercise, but I am thinking about starting within the next 6 months I currently engage in some exercise but not on a regular I currently exercise regularly, but I have only begun within the last 6 months.

I currently exercise regularly, and I have done so for longer than the last 6 months. I choose not to answer

10. I will exercise regularly in the next month

Extremely unlikely 1-6 Extremely likely

I choose not to answer

11. How important is it for you to maintain a healthy active lifestyle

Not important 1-6 Very Important

I choose not to answer

12. On a typical week, in your opinion, how many days is an average WVU student engaged at least in 30 minutes of moderate physical activity?

Days 0 - 7

I choose not to answer

13. On a typical week, in your opinion, how many days is an average WVU student engaged in strength exercise?

Days 0 - 7

I choose not to answer

14. On a typical week, in your opinion, how many days is an average WVU student engaged in at least 20 minutes of vigorous physical activity

Days 0 - 7 
I choose not to answer

15. Which of the following barriers interfere with or prevent you from being physically active?

Lack of time

Yes

No

I choose not to answer

Tiredness

Yes

No

I choose not to answer

Not knowing how to exercise

Yes

No

I choose not to answer

Availability of facilities

Yes

No

I choose not to answer

Lack of friends to exercise with

Yes

No

I choose not to answer

Having more important things to do before exercising

Yes

No

I choose not to answer

Lack of enjoyment in exercising

Yes

No

I choose not to answer

16. On a typical day in the last week, how many times did you eat fruit or drink $100 \%$ fruit juices Do not count punch, Kool-Aid(r), Fruitopia(r), iced tea, Sunny D(r), or sports drinks such as Gatorade(r) or Powerade(r).)?

0 - 7 or more

I choose not to answer

17. On a typical day in the last week, how many times did you eat green salad?

$0-7$ or more

I choose not to answer

18. On a typical day in the last week, how many times did you eat other vegetables (Do not include green salad)

0 - 7 or more 
I choose not to answer

19. On a typical day in the last week, how many ounces of regular pop/soda did you drink? Do not include diet pop/soda. ( 1 vending machine bottle of soda $=20 \mathrm{oz} ; 1 \mathrm{can}=12 \mathrm{oz}$ )

Zero

$1-20 \mathrm{Oz}$

$21-40 \mathrm{Oz}$

$41 \mathrm{Oz}$ or more

I choose not to answer

20. In the last 7 days, how many nights did you eat after $10 \mathrm{pm}$ ?

Days 0 - 7

I choose not to answer

21. In the last 7 days, how many times did you eat Fast food (ex: McDonalds, Kentucky Fried Chicken, Pizza Hut)?

Days 0 - 7

I choose not to answer

22. On a typical day in the last week, how many meals did you have that were provided as part of the meal plan provided through WVU?

Days 0 - 7

I choose not to answer

23. In the last 7 days, how many days did you skip breakfast?

Days 0 - 7

I choose not to answer

24. In the last 7 days, how many days did you skip one of the other two main meals (lunch and dinner)?

Days 0 - 7

I choose not to answer

Eat Healthy = Daily consumption of 2-3 cups of fruit; 2-3cups of vegetables, eat all meals, and avoid late dinners and fast food based meals.

25. Which of these statements most closely reflects you in your eating behavior?

I currently do not eat healthy and I am not thinking about starting within the next 6 months.

I currently do not eat healthy, but I am thinking about starting within the next 6 months.

I currently eat healthy but not on a regular basis

I currently eat healthy regularly, but I have only begun within the last 6 months.

I currently eat healthy, and I have done so for longer than the last 6 months.

I choose not to answer 
26. I will eat healthy in the next month

Definitely unlikely 1-6 Definitely likely

I choose not to answer

27. How important is it for you to maintain a healthy nutritional lifestyle

Not important 1-6 Very Important

I choose not to answer

28. On a typical week, in your opinion, how many days does an average WVU student eat 5 or more serving of fruit or vegetables?

Days 0 - 7

I choose not to answer

29. Which of the following barriers interfere with or prevent you from eating healthy?

Lack of healthy choices available in campus eateries

Yes

No

I choose not to answer

Lack of will power to find healthy food

Yes

No

I choose not to answer

Lack of money to buy healthy food

Yes

No

I choose not to answer

Time constriction to prepare/look for healthy choice

Yes

No

I choose not to answer

Lack of tasty healthy choices

Yes

No

I choose not to answer

Lack of healthy choice in off-campus restaurants

Yes

No

I choose not to answer

Although we understand that you might not be legally allowed to drink because of your age, the purpose of the survey is only to evaluate your health and therefore there are not possible negative repercussion for underage drinking. Please BE COMPLETEY HONEST in answering to the following questions. 1 Drink of Alcohol = $12 \mathrm{oz}$. of beer (can), $5 \mathrm{oz}$. of wine (glass), or $1 \mathrm{oz}$. of liquor (a shot) 
30. In the last 7 days, how many days per week did you have at least one drink of any alcoholic beverage?

Days 0 - 7

I choose not to answer

31. On a typical day in the last week, how many drinks per day did you have?

$0,1-2,3-4,5-6,7-8,9-10$, more than 10

I choose not to answer

32. In the last 7 days, how many times did you drink more than 5 drinks in a sitting

Times 0 - 7

I choose not to answer

33. In last month, how many times did you drink more than 5 drinks in a sitting?

$0,1-3,4-7,8-11,12-15$, more than 15

I choose not to answer

Moderate Drinking $=$ max two drinks a day and no more than 9 drinks per week for women and 12-14 for men

34. Which of these statements most closely reflects you in your drinking behavior?

I currently do not drink with moderation and I am not thinking about starting wthin the next 6 months

I currently do not drink with moderation, but I am thinking about starting within the next 6 months

I currently drink with moderation but not on a regular basis

I am currently changing my drinking, but I have only begun within the last 6 months.

I currently drink moderately (or not drink at all), and I have done so for longer than the last 6 months

I choose not to answer

35. I will drink moderately in the next month

Extremely unlikely 1-6 Extremely likely

I choose not to answer

36. How important is it for you to maintain a moderate drinking

Not important 1-6 Very Important

I choose not to answer

37. In the last month, in your opinion, how many days does an average WVU student drink?

$0,1-2,3-5,6-9,10-19,20-29$, everyday

I choose not to answer

38. In your opinion, how many drinks do you think an average WVU students drinks during a social event?

$0,1,2,3,4,5,6,7,8,9,10,11-20$, more $\tan 20$ 
I choose not to answer

39. In your opinion, how many times does an average WVU student drink more than 5 (4 if a woman) drinks in a single setting in a two week period?

$0,1-2,3-4,5-6,7-8,9-10$, more than 10

I choose not to answer

40. How often do you think that an average WVU student has experienced negative consequences of drinking in the last year?

None

Rarely

Sometimes

Most of the

Always

I choose not to answer

41. Which of the following barriers interfere with or prevent you from drinking in moderation?

Peer Pressure

Yes

No

I choose not to answer

No alternative to drink to manage stress

Yes

No

I choose not to answer

No alternative to drink in order to have fun

Yes

No

I choose not to answer

No alternative to socialize with others

Yes

No

I choose not to answer

Need to fit in

Yes

No

I choose not to answer

42. How would you define yourself?

White (non-Hispanic)

Black (non-Hispanic

Hispanic or Latino

Asian or Pacific Islander

Multi-racial

Other

I choose not to answer 
43. Class status:

Freshmen

Sophomore

Junior

Senior

Transfer

I choose not to answer

44. Where do you currently live

Residence hall

Other university/college housing

Off-campus house or apartment

I choose not to answer

45. How tall are you in inches?

(Type just a period in the text box if you choose not to answer.)

46. How much do you weigh in pounds?

(Type just a period in the text box if you choose not to answer.)

47. Please indicate whether you are a full-time student.

Are you a full-time student?

Yes

No

I choose not to answer

48. Are you participating in any other form of health-related program (B4B, bog looser, etc..) If yes, please enter the approximate date you began participating in the program(s). If not, please enter "No".

(Type just a period in the text box if you choose not to answer.)

49. For me exercising regularly is enjoyable

Definitely false 1-6 Definitely true

I choose not to answer

50. Most people who are important to me regularly exercise

Definitely false 1-6 Definitely true

I choose not to answer 
51 If I wanted to I could start exercising regularly in the next month

Definitely false 1-6 Definitely true

I choose not to answer

52. For me to eat healthy regularly in the forthcoming month is enjoyable

Definitely false 1-6 Definitely true

I choose not to answer

53. People who are important to me eat healthy

Definitely false 1-6 Definitely true

I choose not to answer

54. If I wanted to I could start to eat healthy in the next month

Definitely false 1-6 Definitely true

I choose not to answer

55. For me to drink moderately regularly in the forthcoming month is enjoyable

Definitely false 1-6 Definitely true

I choose not to answer

56. People who are important to me drink moderately

Definitely false 1-6 Definitely true

I choose not to answer

57. If I wanted to I could start to moderate my drinking in the next month

Definitely false 1-6 Definitely true

I choose not to answer 


\section{Administration 2}

Please answer the questions on this page and the next page. Then click at the bottom of the next page to see feedback.

Enter your 700 ID number:

1. Please write your MIX ID (e.g. Andrew Lopez --> alopez)

(Type just a period in the text box if you choose not to answer.)

2. In general, how would you define your lifestyle? Would you say

Very healthy

Somewhat Healthy

Somewhat unhealthy

Very unhealthy

I choose not to answer

3. Your gender:

Male

Female

I choose not to answer

Moderate physical activities are those, like walking, light bicycling, yoga, or Pilates that cause small increases in breathing or heart rate.

4. In a usual week how many days do you do 30 or more minutes of moderate activity (only count bouts of at least 10 minutes)?

Days 0 - 7

I choose not to answer

Vigorous physical activities cause large increases in breathing or heart rate, like running, lifting weights, aerobics, or Zumba.

5. Again, in a usual week, how many days do you do 20 or more minutes of vigorous activity (only count bouts of at least 10 minutes)?

Days 0 - 7

I choose not to answer

6. On how many of the past 7 days did you do stretching exercises, such as toe touching, knee bending, or leg stretching?

Days 0 - 7

I choose not to answer 
7. On how many of the past 7 days did you do exercises to strengthen or tone your muscles, such as push-ups, sit-ups, or weight lifting?

Days 0 - 7

I choose not to answer

8. Where do you normally like to exercise?

Indoor

Outdoor

No Exercise

I choose not to answer

"Regular Exercise" includes activities (such as power walking, jogging, swimming, aerobic dancing, biking, rowing, weight lifting, sports, stretching, etc.) 3 times or more per week for 20 minutes or longer each time. Activities that are primarily sedentary, such as bowling or playing golf with a golf cart, are NOT considered exercise.

9. Which of these statements most closely reflects you in your exercise behavior?

I currently do not engage in exercise and I am not thinking about starting within the next 6 months.

I currently do not exercise, but I am thinking about starting within the next 6 months I currently engage in some exercise but not on a regular I currently exercise regularly, but I have only begun within the last 6 months.

I currently exercise regularly, and I have done so for longer than the last 6 months. I choose not to answer

10. I will exercise regularly in the next month

Extremely unlikely 1-6 Extremely likely

I choose not to answer

11. How important is it for you to maintain a healthy active lifestyle

Not important 1-6 Very Important

I choose not to answer

12. On a typical week, in your opinion, how many days is an average WVU student engaged at least in 30 minutes of moderate physical activity?

Days 0 - 7

I choose not to answer

13. On a typical week, in your opinion, how many days is an average WVU student engaged in strength exercise?

Days 0 - 7

I choose not to answer

14. On a typical week, in your opinion, how many days is an average WVU student engaged in at least 20 minutes of vigorous physical activity

Days 0 - 7 
I choose not to answer

15. Which of the following barriers interfere with or prevent you from being physically active?

Lack of time

Yes

No

I choose not to answer

Tiredness

Yes

No

I choose not to answer

Not knowing how to exercise

Yes

No

I choose not to answer

Availability of facilities

Yes

No

I choose not to answer

Lack of friends to exercise with

Yes

No

I choose not to answer

Having more important things to do before exercising

Yes

No

I choose not to answer

Lack of enjoyment in exercising

Yes

No

I choose not to answer

16. On a typical day in the last week, how many times did you eat fruit or drink $100 \%$ fruit juices Do not count punch, Kool-Aid(r), Fruitopia(r), iced tea, Sunny D(r), or sports drinks such as Gatorade(r) or Powerade(r).)?

0 - 7 or more

I choose not to answer

17. On a typical day in the last week, how many times did you eat green salad?

$0-7$ or more

I choose not to answer 
18. On a typical day in the last week, how many times did you eat other vegetables (Do not include green salad)

$0-7$ or more

I choose not to answer

19. On a typical day in the last week, how many ounces of regular pop/soda did you drink? Do not include diet pop/soda. ( 1 vending machine bottle of soda $=20 \mathrm{oz} ; 1 \mathrm{can}=12 \mathrm{oz}$ )

Zero

$1-20 \mathrm{Oz}$

$21-40 \mathrm{Oz}$

$41 \mathrm{Oz}$ or more

I choose not to answer

20. In the last 7 days, how many nights did you eat after $10 \mathrm{pm}$ ?

Days 0 - 7

I choose not to answer

21. In the last 7 days, how many times did you eat Fast food (ex: McDonalds, Kentucky Fried Chicken, Pizza Hut)?

Days 0 - 7

I choose not to answer

22. On a typical day in the last week, how many meals did you have that were provided as part of the meal plan provided through WVU?

Days 0 - 7

I choose not to answer

23. In the last 7 days, how many days did you skip breakfast?

Days 0 - 7

I choose not to answer

24. In the last 7 days, how many days did you skip one of the other two main meals (lunch and dinner)?

Days 0 - 7

I choose not to answer

Eat Healthy = Daily consumption of 2-3 cups of fruit; 2-3cups of vegetables, eat all meals, and avoid late dinners and fast food based meals.

25. Which of these statements most closely reflects you in your eating behavior?

I currently do not eat healthy and I am not thinking about starting within the next 6 months.

I currently do not eat healthy, but I am thinking about starting within the next 6 months. I currently eat healthy but not on a regular basis

I currently eat healthy regularly, but I have only begun within the last 6 months. I currently eat healthy, and I have done so for longer than the last 6 months. 
I choose not to answer

26. I will eat healthy in the next month

Definitely unlikely 1-6 Definitely likely

I choose not to answer

27. How important is it for you to maintain a healthy nutritional lifestyle

Not important 1-6 Very Important

I choose not to answer

28. On a typical week, in your opinion, how many days does an average WVU student eat 5 or more serving of fruit or vegetables?

Days 0 - 7

I choose not to answer

29. Which of the following barriers interfere with or prevent you from eating healthy?

Lack of healthy choices available in campus eateries

Yes

No

I choose not to answer

Lack of will power to find healthy food

Yes

No

I choose not to answer

Lack of money to buy healthy food

Yes

No

I choose not to answer

Time constriction to prepare/look for healthy choice

Yes

No

I choose not to answer

Lack of tasty healthy choices

Yes

No

I choose not to answer

Lack of healthy choice in off-campus restaurants

Yes

No

I choose not to answer

Although we understand that you might not be legally allowed to drink because of your age, the purpose of the survey is only to evaluate your health and therefore there are not possible negative repercussion for underage drinking. Please BE COMPLETEY HONEST in answering to the 
following questions. 1 Drink of Alcohol = 12 oz. of beer (can), 5 oz. of wine (glass), or $1 \mathrm{oz}$. of liquor (a shot)

30. In the last 7 days, how many days per week did you have at least one drink of any alcoholic beverage?

Days 0 - 7

I choose not to answer

31.On a typical day in the last week, how many drinks per day did you have?

$0,1-2,3-4,5-6,7-8,9-10$, more than 10

I choose not to answer

32. In the last 7 days, how many times did you drink more than 5 drinks in a sitting

Times 0 - 7

I choose not to answer

33. In last month, how many times did you drink more than 5 drinks in a sitting?

$0,1-3,4-7,8-11,12-15$, more than 15

I choose not to answer

Moderate Drinking $=$ max two drinks a day and no more than 9 drinks per week for women and 12-14 for men

34. Which of these statements most closely reflects you in your drinking behavior?

I currently do not drink with moderation and I am not thinking about starting wthin the next 6 months

I currently do not drink with moderation, but I am thinking about starting within the next 6 months

I currently drink with moderation but not on a regular basis

I am currently changing my drinking, but I have only begun within the last 6 months.

I currently drink moderately (or not drink at all), and I have done so for longer than the last 6 months

I choose not to answer

35. I will drink moderately in the next month

Extremely unlikely 1-6 Extremely likely

I choose not to answer

36. How important is it for you to maintain a moderate drinking

Not important 1-6 Very Important

I choose not to answer

37. In the last month, in your opinion, how many days does an average WVU student drink?

$0,1-2,3-5,6-9,10-19,20-29$, everyday

I choose not to answer 
38. In your opinion, how many drinks do you think an average WVU students drinks during a social event?

$0,1,2,3,4,5,6,7,8,9,10,11-20$, more tan 20

I choose not to answer

39. In your opinion, how many times does an average WVU student drink more than 5 (4 if a woman) drinks in a single setting in a two week period?

$0,1-2,3-4,5-6,7-8,9-10$, more than 10

I choose not to answer

40. How often do you think that an average WVU student has experienced negative consequences of drinking in the last year?

None

Rarely

Sometimes

Most of the

Always

I choose not to answer

41. Which of the following barriers interfere with or prevent you from drinking in moderation?

Peer Pressure

Yes

No

I choose not to answer

No alternative to drink to manage stress

Yes

No

I choose not to answer

No alternative to drink in order to have fun

Yes

No

I choose not to answer

No alternative to socialize with others

Yes

No

I choose not to answer

Need to fit in

Yes

No

I choose not to answer

42. Compared to high school do you think that you drink

much less $-2,-1,0,+1,+2$ much more

I choose not to answer 
43.Compared to high school do you think that you eat much worse $-2,-1,0,+1,+2$ much better I choose not to answer

44. Compared to high school do you think that you exercise much less $-2,-1,0,+1,+2$ much more I choose not to answer

45. How are you adjusting to college?

Very poorly $-2,-1,0,+1,+2$ Very well I choose not to answer

46. How attached are you to West Virginia University?

Very little $-2,-1,0,+1,+2$ Very much I choose not to answer

47. How many D or F did you receive during the Fall semester?

0-8

I choose not to answer

48. Your age:

(Type just a period in the text box if you choose not to answer.)

49. How would you define yourself?

White (non-Hispanic)

Black (non-Hispanic

Hispanic or Latino

Asian or Pacific Islander

Multi-racial

Other

I choose not to answer

50. Class status:

Freshmen

Sophomore

Junior

Senior

Transfer

I choose not to answer

51. Where do you currently live

Residence hall

Other university/college housing

Off-campus house or apartment

I choose not to answer 
52. How tall are you in inches?

(Type just a period in the text box if you choose not to answer.)

53. How much do you weigh in pounds?

(Type just a period in the text box if you choose not to answer.)

54. Please indicate whether you are a full-time student.

Are you a full-time student?

Yes

No

I choose not to answer

55. For me exercising regularly is enjoyable

Definitely false 1-6 Definitely true

I choose not to answer

56. Most people who are important to me regularly exercise

Definitely false 1-6 Definitely true

I choose not to answer

57. If I wanted to I could start exercising regularly in the next month

Definitely false 1-6 Definitely true

I choose not to answer

58. For me to eat healthy regularly in the forthcoming month is enjoyable

Definitely false 1-6 Definitely true

I choose not to answer

59. People who are important to me eat healthy

Definitely false 1-6 Definitely true

I choose not to answer

60. If I wanted to I could start to eat healthy in the next month

Definitely false 1-6 Definitely true

I choose not to answer

61. For me to drink moderately regularly in the forthcoming month is enjoyable

Definitely false 1-6 Definitely true

I choose not to answer

62. People who are important to me drink moderately

Definitely false 1-6 Definitely true

I choose not to answer 
63. If I wanted to I could start to moderate my drinking in the next month Definitely false 1-6 Definitely true

I choose not to answer 


\section{Administration 3}

Please answer the questions on this page and the next page. Then click at the bottom of the next page to see feedback.

Enter your 700 ID number:

Enter your 700 ID number again:

1. Please write your MIX ID (e.g. Andrew Lopez --> alopez)

(Type just a period in the text box if you choose not to answer.)

2. In general, how would you define your lifestyle? Would you say

Very healthy

Somewhat Healthy

Somewhat unhealthy

Very unhealthy

I choose not to answer

3. Your gender:

Male

Female

I choose not to answer

Moderate physical activities are those, like walking, light bicycling, yoga, or Pilates that cause small increases in breathing or heart rate.

4. In a usual week how many days do you do 30 or more minutes of moderate activity (only count bouts of at least 10 minutes)?

Days 0 - 7

I choose not to answer

Vigorous physical activities cause large increases in breathing or heart rate, like running, lifting weights, aerobics, or Zumba.

5. Again, in a usual week, how many days do you do 20 or more minutes of vigorous activity (only count bouts of at least 10 minutes)?

Days 0 - 7

I choose not to answer

6. On how many of the past 7 days did you do stretching exercises, such as toe touching, knee bending, or leg stretching?

Days 0 - 7

I choose not to answer 
7. On how many of the past 7 days did you do exercises to strengthen or tone your muscles, such as push-ups, sit-ups, or weight lifting?

Days 0 - 7

I choose not to answer

8. Where do you normally like to exercise?

Indoor

Outdoor

No Exercise

I choose not to answer

"Regular Exercise" includes activities (such as power walking, jogging, swimming, aerobic dancing, biking, rowing, weight lifting, sports, stretching, etc.) 3 times or more per week for 20 minutes or longer each time. Activities that are primarily sedentary, such as bowling or playing golf with a golf cart, are NOT considered exercise.

9. Which of these statements most closely reflects you in your exercise behavior?

I currently do not engage in exercise and I am not thinking about starting within the next 6 months.

I currently do not exercise, but I am thinking about starting within the next 6 months I currently engage in some exercise but not on a regular I currently exercise regularly, but I have only begun within the last 6 months.

I currently exercise regularly, and I have done so for longer than the last 6 months. I choose not to answer

10. I will exercise regularly in the next month

Extremely unlikely 1-6 Extremely likely

I choose not to answer

11. How important is it for you to maintain a healthy active lifestyle

Not important 1-6 Very Important

I choose not to answer

12. On a typical week, in your opinion, how many days is an average WVU student engaged at least in 30 minutes of moderate physical activity?

Days 0 - 7

I choose not to answer

13. On a typical week, in your opinion, how many days is an average WVU student engaged in strength exercise?

Days 0 - 7

I choose not to answer

14. On a typical week, in your opinion, how many days is an average WVU student engaged in at least 20 minutes of vigorous physical activity

Days $0-7$ 
I choose not to answer

15. Which of the following barriers interfere with or prevent you from being physically active?

Lack of time

Yes

No

I choose not to answer

Tiredness

Yes

No

I choose not to answer

Not knowing how to exercise

Yes

No

I choose not to answer

Availability of facilities

Yes

No

I choose not to answer

Lack of friends to exercise with

Yes

No

I choose not to answer

Having more important things to do before exercising

Yes

No

I choose not to answer

Lack of enjoyment in exercising

Yes

No

I choose not to answer

16. On a typical day in the last week, how many times did you eat fruit or drink $100 \%$ fruit juices Do not count punch, Kool-Aid(r), Fruitopia(r), iced tea, Sunny D(r), or sports drinks such as Gatorade(r) or Powerade(r).)?

0 - 7 or more

I choose not to answer

17. On a typical day in the last week, how many times did you eat green salad?

$0-7$ or more

I choose not to answer

18. On a typical day in the last week, how many times did you eat other vegetables (Do not include green salad)

0 - 7 or more 
I choose not to answer

19. On a typical day in the last week, how many ounces of regular pop/soda did you drink? Do not include diet pop/soda. ( 1 vending machine bottle of soda $=20 \mathrm{oz} ; 1 \mathrm{can}=12 \mathrm{oz}$ )

Zero

$1-20 \mathrm{Oz}$

$21-40 \mathrm{Oz}$

$41 \mathrm{Oz}$ or more

I choose not to answer

20. In the last 7 days, how many nights did you eat after $10 \mathrm{pm}$ ?

Days 0 - 7

I choose not to answer

21. In the last 7 days, how many times did you eat Fast food (ex: McDonalds, Kentucky Fried Chicken, Pizza Hut)?

Days 0 - 7

I choose not to answer

22. On a typical day in the last week, how many meals did you have that were provided as part of the meal plan provided through WVU?

Days 0 - 7

I choose not to answer

23. In the last 7 days, how many days did you skip breakfast?

Days 0 - 7

I choose not to answer

24. In the last 7 days, how many days did you skip one of the other two main meals (lunch and dinner)?

Days 0 - 7

I choose not to answer

Eat Healthy = Daily consumption of 2-3 cups of fruit; 2-3cups of vegetables, eat all meals, and avoid late dinners and fast food based meals.

25. Which of these statements most closely reflects you in your eating behavior?

I currently do not eat healthy and I am not thinking about starting within the next 6 months.

I currently do not eat healthy, but I am thinking about starting within the next 6 months.

I currently eat healthy but not on a regular basis

I currently eat healthy regularly, but I have only begun within the last 6 months.

I currently eat healthy, and I have done so for longer than the last 6 months.

I choose not to answer 
26. I will eat healthy in the next month

Definitely unlikely 1-6 Definitely likely

I choose not to answer

27. How important is it for you to maintain a healthy nutritional lifestyle

Not important 1-6 Very Important

I choose not to answer

28. On a typical week, in your opinion, how many days does an average WVU student eat 5 or more serving of fruit or vegetables?

Days 0 - 7

I choose not to answer

29. Which of the following barriers interfere with or prevent you from eating healthy?

Lack of healthy choices available in campus eateries

Yes

No

I choose not to answer

Lack of will power to find healthy food

Yes

No

I choose not to answer

Lack of money to buy healthy food

Yes

No

I choose not to answer

Time constriction to prepare/look for healthy choice

Yes

No

I choose not to answer

Lack of tasty healthy choices

Yes

No

I choose not to answer

Lack of healthy choice in off-campus restaurants

Yes

No

I choose not to answer

Although we understand that you might not be legally allowed to drink because of your age, the purpose of the survey is only to evaluate your health and therefore there are not possible negative repercussion for underage drinking. Please BE COMPLETEY HONEST in answering to the following questions. 1 Drink of Alcohol = $12 \mathrm{oz}$. of beer (can), $5 \mathrm{oz}$. of wine (glass), or $1 \mathrm{oz}$. of liquor (a shot) 
30. In the last 7 days, how many days per week did you have at least one drink of any alcoholic beverage?

Days 0 - 7

I choose not to answer

31.On a typical day in the last week, how many drinks per day did you have?

$0,1-2,3-4,5-6,7-8,9-10$, more than 10

I choose not to answer

32. In the last 7 days, how many times did you drink more than 5 drinks in a sitting

Times 0 - 7

I choose not to answer

33. In last month, how many times did you drink more than 5 drinks in a sitting?

$0,1-3,4-7,8-11,12-15$, more than 15

I choose not to answer

Moderate Drinking $=$ max two drinks a day and no more than 9 drinks per week for women and 12-14 for men

34. Which of these statements most closely reflects you in your drinking behavior?

I currently do not drink with moderation and I am not thinking about starting within the next 6 months

I currently do not drink with moderation, but I am thinking about starting within the next 6 months

I currently drink with moderation but not on a regular basis

I am currently changing my drinking, but I have only begun within the last 6 months.

I currently drink moderately (or not drink at all), and I have done so for longer than the last 6 months

I choose not to answer

35. I will drink moderately in the next month

Extremely unlikely 1-6 Extremely likely

I choose not to answer

36. How important is it for you to maintain a moderate drinking

Not important 1-6 Very Important

I choose not to answer

37. In the last month, in your opinion, how many days does an average WVU student drink?

$0,1-2,3-5,6-9,10-19,20-29$, everyday

I choose not to answer

38. In your opinion, how many drinks do you think an average WVU students drinks during a social event?

$0,1,2,3,4,5,6,7,8,9,10,11-20$, more than 20 
I choose not to answer

39. In your opinion, how many times does an average WVU student drink more than 5 (4 if a woman) drinks in a single setting in a two week period?

$0,1-2,3-4,5-6,7-8,9-10$, more than 10

I choose not to answer

40. How often do you think that an average WVU student has experienced negative consequences of drinking in the last year?

None

Rarely

Sometimes

Most of the

Always

I choose not to answer

41. Which of the following barriers interfere with or prevent you from drinking in moderation?

Peer Pressure

Yes

No

I choose not to answer

No alternative to drink to manage stress

Yes

No

I choose not to answer

No alternative to drink in order to have fun

Yes

No

I choose not to answer

No alternative to socialize with others

Yes

No

I choose not to answer

Need to fit in

Yes

No

I choose not to answer

42. How are you adjusting to college?

Very poorly $-2,-1,0-+1,+2$ Very Well

I choose not to answer

43. How attached are you to West Virginia University?

Very little $-2,-1,0-+1,+2$ Very much

I choose not to answer 
44. How many D or F did you receive during the Fall semester?

0 to 8

I choose not to answer

45. After this first year at WVU, are you considering to

Stay at WVU

Transfer from WVU

Withdraw from WVU

I choose not to answer

46. What was your main reason for making the above decision

(Type just a period in the text box if you choose not to answer.)

47. Are you participating in any other form of health-related program (B4B, bog looser, etc..) If yes, please enter the approximate date you began participating in the program(s). If not, please enter "No".

(Type just a period in the text box if you choose not to answer.)

48. In a typical day, how many times did you log in your Facebook account?

$0,1-2,3-4,5-6,7-8,9-10$, more than 10

I choose not to answer

49. In a typical week, how many times did you log in your FB account?

$0,1-2,3-4,5-6,7-8,9-10$, more than 10

I choose not to answer

50. Did you ever socialize with other students on the WellWVU Facebook page?

$\mathrm{Y}, \mathrm{N}$

I choose not to answer

51. How did you find the content of the WellWVU FB group account

Not at al Useful 0-6 Very useful

I choose not to answer

52. This intervention to improve you health lifestyle seemed to you to be

Very ineffective 0-6 Very effective

I choose not to answer

53. Did you receive an individual feedback?

$\mathrm{Y}, \mathrm{N}$

I choose not to answer 
54. Did you read the feedback?

$\mathrm{Y}, \mathrm{N}$

I choose not to answer

55. Did you think that the feedback you received was useful to you?

$\mathrm{Y}, \mathrm{N}$

I choose not to answer

56. Would you recommend your friend to be part of this intervention next year

$\mathrm{Y}, \mathrm{N}$

I choose not to answer

57. What did you like the most about the intervention

(Type just a period in the text box if you choose not to answer.)

58. What did you dislike the most about the intervention

(Type just a period in the text box if you choose not to answer.)

59. Is there anything that would you change in the intervention

(Type just a period in the text box if you choose not to answer.)

60. For me exercising regularly is enjoyable

Definitely false 1-6 Definitely true

I choose not to answer

61. Most people who are important to me regularly exercise

Definitely false 1-6 Definitely true

I choose not to answer

62. If I wanted to I could start exercising regularly in the next month

Definitely false 1-6 Definitely true

I choose not to answer

63. For me to eat healthy regularly in the forthcoming month is enjoyable

Definitely false 1-6 Definitely true

I choose not to answer

64. People who are important to me eat healthy

Definitely false 1-6 Definitely true

I choose not to answer 
65. If I wanted to I could start to eat healthy in the next month

Definitely false 1-6 Definitely true

I choose not to answer

66. For me to drink moderately regularly in the forthcoming month is enjoyable Definitely false 1-6 Definitely true

I choose not to answer

67. People who are important to me drink moderately

Definitely false 1-6 Definitely true

I choose not to answer

68. If I wanted to I could start to moderate my drinking in the next month

Definitely false 1-6 Definitely true

I choose not to answer 
APPENDIX E: Structure of the Personalized Feedback: Keys and Content

GENERAL HEALTH FEEDBACK

(Q3>=5 or Q4>=3) plus (Q18 plus Q19 plus Q20>=6) plus (Q35<=2) A

According to your answers to the survey it seems that you engage in healthy behaviors and that you are in good health. Although this good health, we can always do better and improve or maintain our healthy habits. This feedback will tell you what you could do better or how to keep maintaining your good health.

(Q3>=5 or $\mathrm{Q} 4>=3)$ plus (Q18 plus Q19 plus Q20>=6) plus (Q35>2) B According to your answers to the survey it seems that you engage in healthy behaviors and that you are in good health. Although this good health, we can always do better and improve or maintain our healthy habits. This feedback will tell you what you could do better or how to keep maintaining your good health. For example you might want to think about limiting or moderating your drinking habits.

$(\mathrm{Q} 3>=5$ or $\mathrm{Q} 4>=3)$ plus $(\mathrm{Q} 18$ plus $\mathrm{Q} 19$ plus $\mathrm{Q} 20<6)$ plus $(\mathrm{Q} 35<=2) \mathrm{C}$ According to your answers to the survey it seems that you engage in healthy behaviors and that you are in good health. Although this good health, we can always do better and improve or maintain our healthy habits. This feedback will tell you what you could do better or how to keep maintaining your good health. For example you might want to think about improving the quality of your dietary habits increasing the amount of fruit and vegetables.

$(\mathrm{Q} 3=>5$ or $\mathrm{Q} 4>=3)$ plus $(\mathrm{Q} 18$ plus $\mathrm{Q} 19$ plus $\mathrm{Q} 20<6)$ plus $(\mathrm{Q} 35>2) \mathrm{D}$

According to your answers to the survey it seems that you engage in healthy behaviors and that you are in good health. Although this good health, we can always do better and improve or maintain our healthy habits. This feedback will tell you what you could do better or how to keep maintaining your good health. For example you might want to think about improving the quality of your dietary habits increasing the amount of fruit and vegetables and limiting or moderating your drinking habits.

$(\mathrm{Q} 3<5$ and $\mathrm{Q} 4<3)$ plus (Q18 plus Q19 plus Q20>=6) plus (Q35<=2) E According to your answers to the survey it seems that you engage in healthy behaviors and that you are in good health. Although this good health, we can always do better and improve or maintain our healthy habits. This feedback will tell you what you could do better or how to keep maintaining your good health. For example you might want to think about increase your level of physical activity in order to improve the quality of your life.

$(\mathrm{Q} 3<5$ and $\mathrm{Q} 4<3)$ plus (Q18 plus $\mathrm{Q} 19$ plus $\mathrm{Q} 20>=6)$ plus $(\mathrm{Q} 35>2) \mathrm{F}$

According to your answers to the survey it seems that you engage in healthy behaviors and that you are in good health. Although this good health, we can always do better and improve or maintain our healthy habits. This feedback will tell you what you could do better or how to keep maintaining your good health. For example you might want to think about increase your level of physical activity and limiting or moderating your drinking habits in order to improve the quality of your life. 
$(\mathrm{Q} 3<5$ and $\mathrm{Q} 4<3)$ plus $(\mathrm{Q} 18$ plus Q19 plus Q20<6) plus $(\mathrm{Q} 35<=2) \mathrm{G}$

According to your answers to the survey it seems that you engage in healthy behaviors and that you are in good health. Although this good health, we can always do better and improve or maintain our healthy habits. This feedback will tell you what you could do better or how to keep maintaining your good health. For example you might want to think about increase your level of physical activity and improving the quality of your dietary habits increasing the amount of fruit and vegetables.

$(\mathrm{Q} 3<5$ and $\mathrm{Q} 4<3)$ plus $(\mathrm{Q} 18$ plus Q19 plus Q20<6) plus $(\mathrm{Q} 35>2) \mathrm{H}$

According to your answers to the survey it seems that you engage in not very healthy behaviors. With the goal of improving the quality of your life, you could look at the follow feedback with the goal of learning some strategies to improve your dietary, to limit your drinking habits, and to increase the level of physical activity in your life.

PA Feedback Questions Q2-Q5

$\mathrm{Q} 3 \geq 5=\mathrm{A}$

$\mathrm{Q} 5 \geq 3=\mathrm{A}$

$\mathrm{Q} 3+\mathrm{Q} 4=4=\mathrm{A}$

$\mathrm{Q} 5 \geq 3=\mathrm{A}$

$\mathrm{Q} 6 \geq 2=\mathrm{A}$

$\mathrm{A}=Q 3 A+Q 4 A[Q 3+Q 4=A]+Q 5 A+Q 6 A \rightarrow$

Well done! Your level of physical activity meets the guidelines suggested by the American College of Sports Medicine in all of its aspects; aerobic, strength, and stretching. With this level of physical activity you will be able to obtain good benefits for your wellness and health!

$\mathrm{B}=Q 3 A+Q 4 A / Q 3+Q 4=A]+Q 5 A+Q 6 B$

Well done! Your level of physical activity meets the guidelines suggested by the American College of Sports Medicine in its aspects of aerobics and stretching. You might want to improve a little of your strength habits. Exercise and improvement of strength has the benefits of being able to increase strength in muscles, tendons and ligaments. Also improved strength has been shown to reduce body fat, while increasing the lean body mass.

$\mathrm{C}=Q 3 A+Q 4 A[Q 3+Q 4=A]+Q 5 B+Q 6 A$

Well done! Your level of physical activity meets the guidelines suggested by the American College of Sports Medicine in its aspects of aerobics and strength. You might want to improve a little of your stretching habits. Stretching has the benefits of increasing flexibility in muscles, tendons, and ligaments. Also, stretching helps to reduce body fat, while increasing lean body mass.

$\mathrm{D}=Q 3 A+Q 4 A / Q 3+Q 4=A]+Q 5 B+Q 6 B$

Well done! Your level of physical activity meets the guidelines suggested by the American College of Sports Medicine in its aspects of aerobics and stretching. You might want to improve a little of your strength and stretching habits. Exercise can improve your strength and flexibility. It is useful to improve the strength and flexibility in muscles, tendons, and ligaments, and the reduction of the body fat, while increasing the lean body mass. 
$\mathrm{E}=Q 3 B+Q 4 A / Q 3+Q 4=A]+Q 5 A+Q 6 A$

Well done! Your level of physical activity meets the guidelines suggested by the American College of Sports Medicine in all its aspects of aerobics, strength, and stretching. With this level of physical activity you will be able to obtain benefits for your wellness and health!

$\mathrm{F}=Q 3 B+Q 4 A / Q 3+Q 4=A]+Q 5 A+Q 6 B$

Well done! Your level of physical activity meets the guidelines suggested by the American College of Sports Medicine in its aspects of aerobics and stretching. You might want to improve a little of your strength habits. Exercise and improved strength has the benefits of increased strength in muscles, tendons, and ligaments. Also, increased strength can reduce body fat, while increases lean body mass.

$\mathrm{G}=Q 3 B+Q 4 A / Q 3+Q 4=A]+Q 5 B+Q 6 A$

Well done! Your level of physical activity meets the guidelines suggested by the American College of Sports Medicine in its aspects of aerobics and strength. You might want to improve a little of your stretching habits. Stretching has the benefits of increasing flexibility in muscles, tendons, and ligaments. Also, stretching helps reduce body fat, while increasing lean body mass.

$\mathrm{H}=Q 3 A+Q 4 B[Q 3+Q 4=A]+Q 5 B+Q 6 B$

Well done! Your level of physical activity meets the guidelines suggested by the American College of Sports Medicine in its aspects of aerobics and stretching. You might want to improve a little of your strength and stretching habits. Exercise and improved strength and flexibility helps to improve the strength and flexibility in muscles, tendons, and ligaments. Also, strength and flexibility cant reduce body fat, while increasing lean body mass.

$\mathrm{I}=Q 3 A+Q 4 B[Q 3+Q 4=A]+Q 5 A+Q 6 A$

Well done! Your level of physical activity meets the guidelines suggested by the American College of Sports Medicine in all aspects; aerobics, strength, and stretching. With this level of physical activity you will be able to obtain benefits for your wellness and health! Being active is a great habit, but remember, increasing the type of aerobic activity from moderate to vigorous can only improve your health and wellness benefits.

$\mathrm{J}=Q 3 A+Q 4 B[Q 3+Q 4=A]+Q 5 A+Q 6 B$

Well done! Your level of physical activity meets the guidelines suggested by the American College of Sports Medicine in its aspects of aerobics and stretching. You might want to improve some of your strength habits. Exercise and improve strength has the benefits of increased strength in muscles, tendons, and ligaments. Also, increased strength may reduce body fat, while increasing lean body mass. Being active is a great habit, but remember, increasing the type of aerobic activity from moderate to vigorous can only improve your health and wellness benefits.

$\mathrm{K}=Q 3 A+Q 4 B[Q 3+Q 4=A]+Q 5 B+Q 6 A$

Well done! Your level of physical activity meets the guidelines suggested by the American College of Sports Medicine in its aspects of aerobics and strength. You might want to improve some of your stretching habits. Stretching has the benefits of increased flexibility in muscles, tendons, and ligaments. Also, stretching helps to reduce body fat, while increasing lean body 
mass. Being active is a great habit, but remember, increasing the type of aerobic activity from moderate to vigorous can only improve your health and wellness benefits.

$\mathrm{L}=Q 3 B+Q 4 A / Q 3+Q 4=A]+Q 5 B+Q 6 B$

Well done! Your level of physical activity meets the guidelines suggested by the American College of Sports Medicine in its aspects of aerobics and stretching. You might want to improve some of your strength and stretching habits. Exercise and improved strength and flexibility helps to improve the strength and flexibility in muscles, tendons, and ligaments. Also, strength and flexibility can reduce body fat, while increasing lean body mass. Being active is a great habit, but remember, increasing the type of aerobic activity from moderate to vigorous can only improve your health and wellness benefits.

$\mathrm{M}=Q 3 B+Q 4 B+(Q 3+Q 4=A)+Q 5 A+Q 6 A \rightarrow$

Well done! Your level of physical activity meets the guidelines suggested by the American College of Sports Medicine in all of its aspects; aerobics, strength, and stretching. With this level of physical activity you will be able to obtain benefits for your wellness and health!

$\mathrm{N}=Q 3 B+Q 4 B+(Q 3+Q 4=A)+Q 5 A+Q 6 B$

Well done! Your level of physical activity meets the guidelines suggested by the American College of Sports Medicine in its aspects of aerobics and stretching. You might want to improve some of your strength habits. Exercise and improved strength has benefits of increased strength in muscles, tendons, and ligaments. Also, increased strength reduces body fat, while increasing lean body mass.

$\mathrm{O}=Q 3 B+Q 4 B+(Q 3+Q 4=A)+Q 5 B+Q 6 A$

Well done! Your level of physical activity meets the guidelines suggested by the American College of Sports Medicine in its aspects of aerobics and strength. You might want to improve some of your stretching habits. Stretching has the benefits of increasing flexibility in muscles, tendons, and ligaments. Also, stretching can help reduce body fat, while increasing lean body mass.

$\mathrm{P}=Q 3 B+Q 4 B+(Q 3+Q 4=A)+Q 5 B+Q 6 B$

Well done! Your level of physical activity meets the guidelines suggested by the American College of Sports Medicine in its aspects of aerobics and stretching. You might want to improve some of your strength and stretching habits. Exercise and improved strength and flexibility can improve strength and flexibility in muscles, tendons, and ligaments. Also, strength and flexibility can reduce body fat, while increasing lean body mass.

$\mathrm{Q}=Q 3 B+Q 4 B+(Q 3+Q 4=B)+Q 5 A+Q 6 A \rightarrow$

Well done! Your level of physical activity meets the guidelines suggested by the American College of Sports Medicine in all of its aspects; aerobics, strength, and stretching. With this level of physical activity you will be able to obtain benefits for your wellness and health!

$\mathrm{R}=Q 3 B+Q 4 B+(Q 3+Q 4=B)+Q 5 A+Q 6 B$

Your level of physical activity does not meet the guidelines suggested by the American College of Sports Medicine in any of its aspects, producing negative affects on your health and wellness. 
You might want to improve some of your aerobic activity and strength habits. Aerobic Exercise has beneficial consequences on your cardiovascular/cardiorespiratory functions (heart and lungs), increased blood supply to your muscles and the ability to use oxygen more efficiently (which will help you to exercise more efficiently). Aerobic activity will also lower your heart rate and blood pressure. At any level of sub-maximal exercise, it will increase the threshold for lactic acid accumulation. Exercise and improved strength has the benefits of increased strength in muscles, tendons, and ligaments. Also, increased strength can reduce body fat, while increasing lean body mass.

$\mathrm{S}=Q 3 B+Q 4 B+(Q 3+Q 4=B)+Q 5 B+Q 6 A$

Your level of physical activity does not meet the guidelines suggested by the American College of Sports Medicine in any of its aspects, negatively affecting your health and wellness. You might want to improve some of your aerobic activity and stretching habits. Aerobic Exercise has beneficial consequences on your cardiovascular/cardiorespiratory functions (heart and lungs), increased blood supply to muscles and the ability to use oxygen more efficiently (which will help you to exercise more efficiently). It will also lower your heart rate and blood pressure at any level of sub-maximal exercise. Aerobic activity can also increase the threshold for lactic acid accumulation. Stretching has the benefits of increased flexibility in muscles, tendons, and ligaments. Also, Stretching can help reduce body fat, while increasing lean body mass.

$\mathrm{T}=Q 3 B+Q 4 B+(Q 3+Q 4=B)+Q 5 B+Q 6 B$

Your level of physical activity does not meet the guidelines suggested by the American College of Sports Medicine in any of its aspects, negatively affects your health and wellness. You might want to improve some of your aerobic, strength and stretching activities and habits. Aerobic Exercise has beneficial consequences on your cardiovascular/cardiorespiratory functions (heart and lungs), increases blood supply to muscles and the ability to use oxygen more efficiently (which will help you to exercise more efficiently). It will also lower your heart rate and blood pressure at any level of sub-maximal exercise. It can also increase the threshold for lactic acid accumulation.

Exercise, improved strength and flexibility is useful to improve strength and flexibility in muscles, tendons, and ligaments. Strength and flexibility also can reduce body fat, while increasing lean body mass.

Question Q7

\section{$\underline{\text { Indoor }} \rightarrow$}

The Student Recreation Center and Stansbury Hall are two great facilities offered by the university where you can dedicate time to exercise. Many other private facilities are available in Morgantown.

$\underline{\text { Outdoor }} \rightarrow$

Morgantown offers great opportunities to begin physically activity. The rail trail, the coliseum, the stadium, and other places in Morgantown represent good places to walk, run, swim or practice any type of physical activity. 
$\underline{T T M}$

Feedback to Q8

$1 \rightarrow$ Precontemplation

Even though right now it is hard to understand all the pros of being physically active, there are many positive outcomes that long-term physical activity can provide to your health and wellness. Aerobic Exercise has many cardiovascular/cardiorespiratory (heart and lungs) benefits, along with many other health benefits. However, aerobic exercise is not enough. Strength and stretching training also have many benefits such as an increase in strength and flexibility of muscles, tendons, and ligaments. The also help in reducing body fat, while increasing lean body mass. In order to be physically active, you do not necessarily need to be attending classes or exercising at the Student Rec Center. Instead, you can be physically active during your daily routine (choosing to use stairs instead of elevators or walking to class instead of drive). Some other options to fit your regular schedule are talking on the phone while walking, walking your dog or going to take a walk with friends instead of staying on the couch.

$2 \rightarrow$ Possible Options

Looking at some of the pros of exercising and being active, you may notice that being active can help your cardiovascular/cardiorespiratory systems. Also, you may find an increase in your strength and flexibility of muscles, tendons, and ligaments, while reducing body fat and increasing lean body mass. These positive outcomes can be beneficial to your health and wellness on the long and short term. Being physically active does not necessarily requires you to go to the gym, but you can be physically active by making active decisions like walking instead of driving, walking up stairs instead of using the elevator or walking while talking on the phone. Some frustration at the beginning is normal, but remember that even when you're not noticing any visible benefits of exercise, your body is still becoming healthier. Thirty minutes of moderate exercise five days a week will make your life longer and healthier. Lack of time or motivation are barriers you might experience at the beginning of this process. Sharing these experiences with others will normalize your feelings because you will find others that might have the same experiences as you.

$3 \rightarrow$ Benefits of being consistent (Strategies to be consistent)

Being physically active does not necessarily require the gym, but you can be physically active by making active choices such as walking instead of driving, taking the stairs instead of using the elevator or walking while talking on the phone. Some frustration at the beginning is normal but remember that even when you're not noticing any visible benefits of exercise, your body is still becoming healthier. Thirty minutes of moderate exercise five days a week will make your life longer and healthier. Lack of time or motivation are barriers you might experience at the beginning of this process. Sharing these experiences with others will normalize your feelings because you will find others that might have the same experiences as you. Exercising consistently is fundamental to obtain all possible benefits. It does not entail spending hours doing strenuously active. Regular exercise can be from on short sessions (10 minutes a the time) accumulated along the day. Planning your schedule and make physical activity part of your daily routine will make this behavioral change so much easier in your life. Planning is often more important than motivation. 
$4 \rightarrow$ Strategies to keep going

Lack of time or motivation are barriers you might experience at the beginning of this process. Sharing these experiences with others will normalize your feelings because you will find others that might have the same experiences as you. Exercising consistently is fundamental to obtain all possible benefits. It does not entail spending hours doing strenuously active. Regular exercise can be built from short sessions (10 minutes a the time) accumulated along the day. Planning your schedule and make physical activity part of your daily routine will make this behavioral change so much easier in your life. Planning is often more important than motivation. It is also important to substitute the inactive moments with active alternatives (walking while talking on the phone). More importantly, it could be extremely helpful to find some friends or family members willing to partake in these active with you.

\section{$5 \rightarrow$ Maintenance}

You are doing great! Find some way to reward all this effort you are putting in maintaining these great healthy habits! Rewarding yourself is a good strategy to keep you motivated in maintaining this habit. Remember that you don't need to go to the gym to be active. You can transform your life in general toward a more physically active life by making "active choices" (walking instead of driving). You will receive many benefits to your health! Looking for people to share this life choice can be useful for you in maintaining this habit in a more social way.

Question Q8

$Q 9 \leq 4 \rightarrow Q 10+Q 11+Q 12$

$Q 9 \leq 4$ and/or $Q 13 \leq 4$

Exercising is a very important part of your health. Being consistent in your exercise habits can produce short-term benefits such as loosing weight and feeling more fit. Long term benefits may consist of decreasing heath risks (such as heart problems and obesity) and improving the quality of your life.

$Q 9>4$

Well done! It seems that being physical active is very important to you!!!

$Q 14+Q 15+Q 16 \rightarrow$ In any case give WVU Statistics

At WVU, 22\% of the students do not engage in any moderate physical activity, $40 \%$ do not engage in vigorous physical activity and over $50 \%$ of the students do not engage in any strength exercises. Although the health behavior requires at least three to five days a week of physical activity (vigorous or moderate), only $30 \%$ of the students engage in vigorous activity and $50 \%$ of the students engage in moderate activity. In terms of exercise focused on strength, although the guidelines suggest exercising three times a week, only $25 \%$ of the students engage in such behaviors.

Q17

a. $\underline{Y e s}$ If you sit and write down your schedule, you will realize that you will be able to find half an hour a day to be physically active in your week. Planning is more important than motivation. 
b. $\underline{\text { Yes }}$ The hardest part is the beginning. Start slow and be consistent. With time, fatigue will be limited and you will start to realize the great benefits of being physically active.

c. Yes At the rec center you can find good support in learning how to exercise. Similar information can be found on the web at the WellWVU website.

d. $\underline{\text { Yes }}$ In Morgantown there are many opportunities to exercise. The Student Recreation Center offers great opportunities of exercise. The trail is great for walking, running or skating and the coliseum or the stadium offer great places to walk and socialize with others. At White Park you can find Baseball fields and an ice-rink. Close to Towers you can find tennis courts and a basketball court. On the top floor of the Mountain lair you can find a nice field to play soccer, football, Frisbee, and a court to play basketball. Sport clubs are another option where students can become more physically active.

e. $\underline{\text { Yes }}$ You might be able to find good exercise buddies at the RecCenter, your dorm or at WellWVU Facebook page. Having an exercise partner when starting exercise can be a great motivational tool.

f. $\underline{Y e s}$ Regular exercise (aerobic, strength and stretching) can improve your academic performance. It can help you maintain your weight and feel/look fit. On the long-term it can also help you to improve the quality of your life and make life longer.

g. $\underline{\text { Yes }}$ Being physically active does not necessarily mean going to the gym. You can involve physical activity in your daily life by walking to class or listening to your music while running. Because of this flexibility, the best way to be physically active is to make it fun. For example, joining some friends or some exercise buddies during the "active moments" can make your exercise experience much more enjoyable. Instead of going to the gym, a fun way to exercise can involve playing some games that require active behaviors. Don't forget to reward yourself with some healthy treats.

\section{Nutrition}

$\mathrm{Q} 18 \geq 2+(\mathrm{Q} 19+\mathrm{Q} 20<3)$

Well done! You eat the suggested number of servings of fruit in a day. Continuing with this habit will have a beneficial effect on your health. You might want to increase the number of vegetable servings per day. A total amount of five or more servings of fruit and vegetables will help your health and wellness.

$\mathrm{Q} 18 \geq 2+(\mathrm{Q} 19+\mathrm{Q} 20 \geq 3)$

Well done! You eat the suggested number of servings of fruit and vegetables in a day.

Continuing with this habit will have a beneficial effect on your health and wellness

$\mathrm{Q} 18<2+(\mathrm{Q} 19+\mathrm{Q} 20<3)$

According to your answers you do not eat enough fruits and vegetables. The right amount of servings is considered to be 5 or more servings between the two classes of food. Fruits and vegetables are some of the healthiest and best tasting foods. They are low in sodium and calories, and most are fat free. They help your body get the vitamins, minerals, fiber, and other natural substances it needs to stay healthy.

$\mathrm{Q} 18<2+(\mathrm{Q} 19+\mathrm{Q} 20 \geq 3)$

Well done! You eat the suggested number of servings of vegetables in a day. Continuing with this habit will have a beneficial effect on your health. You might want to increase the number of 
fruit servings per day. A total amount of five or more servings of fruit and vegetables will help your health and wellness.

$\mathrm{Q} 18+\mathrm{Q} 19+\mathrm{Q} 20 \geq 6$

You eat the suggested number of servings of the combination between fruit and vegetables in a day. Continuing with this habit will have beneficial effect on your health. A total amount of six or more servings of fruit and vegetables will help your health and wellness. Fruits and vegetables are some of the healthiest and best tasting foods. They are low in sodium and calories, and most are fat free. They help your body get the vitamins, minerals, fiber, and other natural substances it needs to stay healthy.

$\mathrm{Q} 21 \neq 0 \rightarrow$ feedback (alternatives + negative effects of no diet pops)

Drinking "sugar based" drinks (soda and pops) can be detrimental for your general health. They will produce a non-needed amount of sugar in your body, they also are a source of substantial caloric intake, also they can negatively affect your sugar blood level, with long term consequences. You can buy some "sugar free" options and keep them in your room or chose them at the bars instead of the "normal" sodas

Q22 $\geq 1 \rightarrow$ feedback (negative effects of eating late)

Eating late meals, besides being generally characterized by a poor quality of food, can also produce difficult control of caloric intake, difficult digestion, and a difficult burning process of the calories that can increase the chance of a gain weight.

Q23 $\geq 1 \rightarrow$ feedback (negative effects of eating fast food + alternatives)

Eating in Fast Food restaurants, can produce negative effects on your health. Fast-food produces an enormous amount of calories that can be difficult to burn in a day, they also have issues producing a sense of satiety or feeling of fullness due to the enormous amount of sugars and fats, and the high amount of calories are also addictive. You can try to buy healthy choices to eat in your room, or go to restaurants with healthier choices, such as salads, fruit servings (without caramel), etc.

Q25 $\geq 1 \rightarrow$ feedback (Benefits of eating breakfast + possible quick alternatives)

Skipping breakfast is one of the worst nutritional habits you can have! Breakfast is the most important meal of the day. Eating breakfast will help you fill full during the day and avoid feeling hungry during the day and needing to eat snack at all hours. Doing this can lower the total daily caloric intake and help in trying to maintain a healthy weight. People who do not eat breakfast often have a hard time concentrating. Since we are in class to learn we need to be able to concentrate and focus and a good healthy breakfast can help in improving our academic performance. For a healthy breakfast, consider a cup of coffee or skim milk, a piece of fruit, and whole brain bread with some low sugar jelly and some yogurt. You can find all of these at the WVU cafeteria.

Q25 + Q26 $\geq 1 \rightarrow$ feedback (Benefits of eating all meals)

Skipping meals can have negative effects on your health. Eating a smaller amount of food at all meals, instead of skipping meals, can deeply help in maintaining a healthy weight and in maintaining well balanced general health. It will help you feel ready to go, and never feel too 
heavy because of the food you ate. Little portions can help you eat healthier, and more often. Eat slowly and try to always include fruit and vegetables in your diet.

$\underline{T T M}$

Q27

$1 \rightarrow$ Precontemplation

Although you are not thinking about changing your behaviors toward to a healthier lifestyle, you might want to read about some of the benefits that health nutrition can produce in your life.Fruits and vegetables are some of the healthiest and best tasting foods. They are low in sodium and calories, and most are fat free. Helping your body get the vitamins, minerals, fiber, and other natural substances it needs to stay healthy and to promote good health. People who eat generous amounts of fruit and vegetables as part of a healthful diet, are likely to have reduced risk of chronic diseases, including stroke, other cardiovascular diseases, and certain cancers. They also maintain a healthier body weight, helping you to look and feel good. Easy to eat on-the-go fruits and vegetables can be very helpful in meeting the needs of your busy academic life, reducing your hunger in a healthy way. Fruits and vegetables are a natural source of energy and give the body many nutrients it needs to keep going. Substituting higher-calorie foods with fruits and vegetables for can a weight loss strategy.

Late dinners and fast food based dinner besides being detrimental for your weight, are also unhealthy because our body is not able to metabolize all the nutrients late at night. Early and healthy dinners (and meals in general) can help you feel better and look better. Substituting regular soda drinks with sugar free drinks, will lower the total amount of calories and will reduce the risk of high levels of sugar in your blood, on top of helping you regulate your body weight.

$2 \rightarrow$ Contemplation

If you are thinking about changing, you might want to consider the many benefits that this healthy habit will produce on your life. Fruits and vegetables are some of the healthiest and best tasting foods. They are low in sodium and calories, and most are fat free. Helping your body get the vitamins, minerals, fiber, and other natural substances it needs to stay healthy and to promote good health. People who eat generous amounts of fruit and vegetables, as part of a healthful diet, are likely to have reduced risk of chronic diseases, including stroke and other cardiovascular diseases, and certain cancers. They also maintain a healthier body weight, helping you look and feel good. Easy to eat on-the-go fruits and vegetables can be very helpful in meeting the needs of your busy academic life, reducing your hunger in a healthy way. Fruits and vegetables are a natural source of energy and give the body many nutrients it needs to keep going. Substituting higher-calorie foods with fruits and vegetables for can a weight loss strategy.

Late dinners and fast food based dinner besides being detrimental for your weight, are also unhealthy because your body is not able to metabolize all the nutrients late at night. Early and healthy dinners (and meals in general) can help you to feel better and look better. Substituting regular soda drinks with sugar free drinks, will lower the total amount of calories and will reduce the risk of high levels of sugar in your blood, on top of helping you to regulate your body weight. Some difficulties and frustration in adding fruit and vegetables to our diet is common. They cover a very broad range of tastes and costs. Find those that fit best in your diet and your own preferences. Look for seasonal fruits and vegetables, that are not too expensive and you can also find them in the cafeterias. Fruit and vegetables are extremely good as sources of nutrients and they also help make us feel satiated. 
There are many barriers to a healthy diet, such as lack of time to look for affordable options, money, and motivation are a normal experiences for anyone who decides to improve this habit. Planning early dinners, or organizing yourself with take-a-way healthy lunches, or looking for cheaper healthier option (seasonal fruit) can be a great way to overcome this barriers. Remember that planning is often more important than motivation.

$3 \rightarrow$ Preparation

Although you are thinking about changing, some thoughts are keeping you away from making the change. Some difficulties and frustration in adding fruits and vegetables to out diet is common. They cover a very broad range of taste and cost. Find those that fit in your diet and your own personal tastes. Look for seasonal fruit and vegetables, they are not as expensive and you can also find them in the cafeterias. Fruit and vegetables are extremely good sources of nutrients and they also help make us feeling satiated.

Experiencing some barriers to a healthy diet, such as lack of time to look for affordable options, money, and motivation are a normal experiences for anyone who decides to improve this habit. Planning early dinners, or organizing yourself with take-a-way healthy lunches, or looking for cheaper healthier option (seasonal fruit) can be a great way to overcome these barriers. Remember that planning is often more important than motivation.

\section{$4 \rightarrow$ Action}

Although you already started changing your behaviors, being consistent with this habit is fundamental to improve your health and wellness. Some difficulties and frustration in adding fruit and vegetables to your diet is common. They cover a very broad range of tastes and costs. Find those that better fit in your diet and in your individual choice. Looking for seasonal fruit and vegetables, they are not very expensive and you can also find them in the cafeterias. Fruit and vegetables are extremely good sources of nutrients and they also help make you feel full. Rewarding your self is an important part of your changing behavior. Finding something that could reward your efforts to be healthy can be a great strategy. It can be an activity, such as going to the movie, or a healthier option of a type food that it is not normally considered healthy (low sugar low fat ice cream, for example). Finding healthy alternatives to your unhealthy habits, is a great strategy to being consistent with this change, A good way to eat more fruits and vegetables is to make salads. You can have a variety of each and eat them in the same meal. In order to substitute your late dinner habits, you can try to eat earlier and leave a healthy, and tasty, snack for later.

Even when you have already made the change, it can be hard to avoid unhealthy food, when they surround you. Creating a healthy environment, for example only having healthy snacks in your room, is a great way to support yourself in this change. To avoid fast food and late dinners, you can find healthy alternative (i.e., salads) in these places, or you can try to look for healthier place to eat.

Surrounding yourself with friends that are willing to pay attention to their diet is a great idea. Together you can plan early dinners and you can cook in turns, avoiding fast food habits and not having to cook at night. Together you can share healthy options and snacks according to the taste of each of you. 


\section{$5 \rightarrow$ Maintenance}

Great! You already maintain healthy nutrition and you have been consistent with it for six or more months! Well Done! Below there are some strategies that you can use to keep being consistent with this change!

Rewarding your self is an important part of your changing behavior. Finding something that could reward your effort to be healthy can be a great strategy. It can be an activity, such as going to the movie, or a healthier option of a type food that it is not normally considered healthy (low sugar low fat ice cream, for example). Finding healthy alternatives to your unhealthy habits, is a great strategy to being consistent with this change, A good way to eat fruit and vegetables is to make salads. You can have a variety of them and eat them in the same meal. In order to substitute your late dinner habits, you can try to eat earlier and leave a healthy, and tasty, snack for later.Even after you make the change, it s hard to avoid unhealthy foods, when they surround you . Creating a healthy environment, for example having only healthy snacks in your room, is a great way to support yourself in this change. To avoid fast food and late dinners, you can find a healthy alternative (i.e., salads) in these places, or you can try to look for a healthier place to eat. Surrounding your self with friends that are willing to pay attention to their diet is a great idea. Together you can plan early dinners and you can cook in turns, avoiding the fast food habits and not having to cook at night. Together you can share healthy options and snacks according to the taste of each of you.

\section{Intention to Behave}

$Q 28 \leq 4 \rightarrow Q 29+Q 30+Q 31$

$Q 32 \leq 4 \rightarrow$ Feedback about importance of healthy diet

A healthy diet will help you increase your health and longevity. This healthy habit could also help you have more energy to be more productive in your life, leaving you the ability to do more things that you enjoy. A healthy diet can produce great health benefits in a short time, such as losing weight and feeling fitter, and in the long run decreasing heath risks (such as heart problems and obesity) and improving the quality of your life.

Q32>4

Well done! It seems that healthy eating is already an important part of your life

$Q 33 \rightarrow$ In any case give:

Although the need for fruits and vegetables is known by everybody at WVU only $5 \%$ of the student body eats the recommended amount of fruit and vegetables or does not eat anything. Whereas the $64 \%$ of those studied only eat 1 or 2 servings, and only $26 \%$ eat between 3 and 4 servings.

Q34 if Yes $\rightarrow$ Feedback for each barrier a. list of places to eat healthy on campus

Within campus you can find some healthier options in almost all the restaurants and cafeterias. Some of them are definitely healthier than others such as Hatfields, McCoys, and Sbarro in the Mountainlair or some of the restaurants in town that accept your meal plan.

b. will power is overrated $\rightarrow$ surround yourself with healthy food 
Often time we overrate our willpower. Planning is often more important than motivation. You want to surround yourself with healthy choices and "healthier" friends, and also limit the presence of unhealthy choices around you.

c. cheap options to eat healthy

Often time, money matters when finding healthy options. In the cafeteria we can find few of them, but we can definitely look for more options in the grocery store, looking for cheap fruits and vegetables such as bananas or green salad and many other options. In town there are many different places where you can find these elements for a more than reasonable price. Another idea is to buy seasonal fruit and vegetables (for examples oranges during winter and cantaloupe during summer), it will help keep both your body and your wallet healthy.

d. simple stuff to have in your room instead of bad food

Surround your self with healthy food, for example keeping them in your room will help you always have food ready to go that won't be detrimental for your general health and won't require preparation time.

e. sources for tasty healthy food

Any type of fruit juice (not punches), and vegetables, low fat and low sugar food can be a good option to keep around yourself to provide a healthy source of energy for your day.

f. list of places to eat healthy off campus

In Morgantown there are some healthy options, in terms of restaurants. Some of the most well known among the chain restaurants, are Subway and Panera, but also other options are present, such as Zenclay Café and BlackBear where you can also find some healthy options.

\section{$\underline{\text { Alcohol }}$}

Feedback Questions Q34-Q36

Male

Q34 0=A; 1-2 = B; 3-4 = C; 5-10=D

$\mathrm{Q} 35 \leq 2=\mathrm{A} ; \geq 3=\mathrm{B}$

$\mathrm{Q} 360=\mathrm{A} ; 1-4=\mathrm{B} ; \geq 5=\mathrm{C}$

$\mathrm{Q} 34=\mathrm{A}$

Good Job! Sobriety is a good habit! Alcohol consumption can have many negative consequences on your health directly and indirectly.

$\mathrm{AM}=Q 34 B+Q 35 A+Q 36 A$

Very well done! Drinking moderately is a good habit or at least a good way to not over consume it, being exposed to the negative consequences of it. Moderate drinking and drinking in general definitely has a caloric intake effect, and potentially health effects that need to be kept in consideration.

$\mathrm{BM}=Q 34 B+Q 35 B+Q 36 A$

Even if you generally drink moderately. Drinking in general definitely has a caloric intake effect, and potentially health effects that need to be kept in consideration.

Drinking three or more times in a week is considered frequent drinking. This type of behavior has negative effects on your health on the long and short terms. Physically alcohol is a source of direct and indirect caloric intake, it can also affect your academic performance and it can 
potentially have influence on your safety, because of unhealthy consequences, such as drinking and driving or unsafe sex.

$\mathrm{CM}=Q 34 B+Q 35 A+Q 36 B$

Even if you generally drink moderately. Drinking in general definitely has a caloric intake effect, and potentially health effects that need to be kept in consideration.

Drinking 5 or more drinks per sitting is defined "Binge Drinking", which definitely is an unhealthy behavior. This type of behavior has negative effects on your health on the long and short terms. Physically, alcohol is a source of direct and indirect caloric intake, it can also affect your academic performance and it can potentially have influence on your safety, because of unhealthy consequences, such as drinking and driving or unsafe sex. Remember that not many people is able to totally absorb this amount of alcohol. Also when you feel to be ok, your boy not necessarily has absorbed all the consumed alcohol.

$\mathrm{DM}=Q 34 B+Q 35 B+Q 36 B$

Even if you generally drink moderately. Drinking in general definitely has a caloric intake effect, and potentially health effects that need to be kept in consideration.

Drinking three or more times in a week is considered frequent drinking. This type of behavior has negative effects on your health on the long and short terms. Physically alcohol is a source of direct and indirect caloric intake, it can also affect your academic performance and it can potentially have influence on your safety, because of unhealthy consequences, such as drinking and driving or unsafe sex.

Drinking 5 or more drinks per sitting is defined "Binge Drinking", which definitely is an unhealthy behavior. This type of behavior has negative effects on your health on the long and short terms. Physically, alcohol is a source of direct and indirect caloric intake, it can also affect your academic performance and it can potentially have influence on your safety, because of unhealthy consequences, such as drinking and driving or unsafe sex. Remember that not many people are able to totally absorb this amount of alcohol. Also when you feel to be ok, your boy not necessarily has absorbed all the consumed alcohol.

$\mathrm{EM}=Q 34 B+Q 35 A+Q 36 C$

Even if you generally drink moderately. Drinking in general definitely has a caloric intake effect, and potentially health effects that need to be kept in consideration.

Experiencing five or more episodes of Binge Drinking (5 or more drinks in a setting) in a month is considered to be "Heavy Drinking". This type of behavior has negative effects on your health on the long and short terms. Physically, alcohol is a source of direct and indirect caloric intake, it can also affect your academic performance and it can potentially have influence on your safety, because of unhealthy consequences, such as drinking and driving or unsafe sex. Remember that not many people are able to totally absorb this amount of alcohol. Also when you feel to be ok, your boy not necessarily has absorbed all the consumed alcohol.

$\mathrm{FM}=Q 34 B+Q 35 B+Q 36 C$

Even if you generally drink moderately. Drinking in general definitely has a caloric intake effect, and potentially health effects that need to be kept in consideration.

Although drinking three or more times in a week is already considered to be "Frequent Drinking", experiencing five or more episodes of Binge Drinking (5 or more drinks in a setting) 
in a month is considered to be "Heavy Drinking". This type of behavior has negative effects on your health on the long and short terms. Physically, alcohol is a source of direct and indirect caloric intake, it can also affect your academic performance and it can potentially have influence on your safety, because of unhealthy consequences, such as drinking and driving or unsafe sex. Remember that not many people are able to totally absorb this amount of alcohol. Also when you feel to be ok, your boy not necessarily has absorbed all the consumed alcohol.

\section{$\mathrm{GM}=Q 34 C+Q 35 A+Q 36 A$}

Although the general impression can be that drinking three or four drinks per setting can be considered "Moderate Drinking", it is considered "Exaggerated Drinking". This type of behavior has negative effects on your health on the long and short terms. Drinking moreover having effects on the general daily caloric intake, it can also affect your academic performance and it can potentially influence your health.

$\mathrm{HM}=Q 34 C+Q 35 B+Q 36 A$

Although the general impression can be that drinking three or four drinks per setting can be considered "Moderate Drinking", it is considered "Exaggerated Drinking". In addition to the quantity of alcohol also the frequency of this drinking can be negatively affecting your life. Drinking three or more times in a week is considered "Frequent Drinking".

This type of behavior has negative effects on your health on the long and short terms. Drinking moreover having effects on the general daily caloric intake, it can also affect your academic performance and it can potentially influence your health.

$\mathrm{JM}=Q 34 C+Q 35 A+Q 36 B$

Although the general impression can be that drinking three or four drinks per setting can be considered "Moderate", it is better defined as "Exaggerated Drinking", and when characterized by episodes during which you drink 5 or more drinks per sitting is defined "Binge Drinking", which definitely is an unhealthy behavior. This type of behavior has negative effects on your health on the long and short terms. Physically, alcohol is a source of direct and indirect caloric intake, it can also affect your academic performance and it can potentially have influence on your safety, because of unhealthy consequences, such as drinking and driving or unsafe sex. Remember that not many people are able to totally absorb this amount of alcohol. Also when you feel to be ok, your boy not necessarily has absorbed all the consumed alcohol.

$\mathrm{KM}=Q 34 C+Q 35 B+Q 36 B$

Although the general impression can be that drinking three or four drinks per setting can be considered "Moderate", it is better defined as "Exaggerated Drinking", and when characterized by episodes during which you drink 5 or more drinks per sitting is defined "Binge Drinking", which definitely is an unhealthy behavior. In addition to the quantity of alcohol also the frequency of this drinking can be negatively affecting your life. Drinking three or more times in a week is considered "Frequent Drinking".

Physically, alcohol is a source of direct and indirect caloric intake, it can also affect your academic performance and it can potentially have influence on your safety, because of unhealthy consequences, such as drinking and driving or unsafe sex. Remember that not many people are able to totally absorb this amount of alcohol. Also when you feel to be ok, your boy not necessarily has absorbed all the consumed alcohol. 
$\mathrm{LM}=Q 34 C+Q 35 A+Q 36 C$

Although the general impression can be that drinking three or four drinks per setting can be considered "Moderate", it is better defined as "Exaggerated Drinking", and when characterized by episodes during which you drink 5 or more drinks per sitting is defined "Binge Drinking", which definitely is an unhealthy behavior. When these episodes repeat five or more time in a month, you fit in the category of the "Heavy Drinkers".

Physically, alcohol is a source of direct and indirect caloric intake, it can also affect your academic performance and it can potentially have influence on your safety, because of unhealthy consequences, such as drinking and driving or unsafe sex. Remember that not many people are able to totally absorb this amount of alcohol. Also when you feel to be ok, your boy not necessarily has absorbed all the consumed alcohol.

$\mathrm{MM}=Q 34 C+Q 35 B+Q 36 C$

Although the general impression can be that drinking three or four drinks per setting can be considered "Moderate", it is better defined as "Exaggerated Drinking", and when characterized by episodes during which you drink 5 or more drinks per sitting is defined "Binge Drinking", which definitely is an unhealthy behavior. When these episodes repeat five or more time in a month, you fit in the category of the "Heavy Drinkers". In addition to the quantity of alcohol also the frequency of this drinking can be negatively affecting your life. Drinking three or more times in a week is considered "Frequent Drinking".

Physically, alcohol is a source of direct and indirect caloric intake, it can also affect your academic performance and it can potentially have influence on your safety, because of unhealthy consequences, such as drinking and driving or unsafe sex. Remember that not many people are able to totally absorb this amount of alcohol. Also when you feel to be ok, your boy not necessarily has absorbed all the consumed alcohol.

$\mathrm{NM}=Q 34 D+Q 35 A+Q 36 A \rightarrow I M P O S S I B L E$

$\mathrm{OM}=Q 34 D+Q 35 B+Q 36 A \rightarrow I M P O S S I B L E$

$\mathrm{PM}=Q 34 D+Q 35 A+Q 36 B$

Even if you do not have the perception of having an uncommon drinking habits, drinking 5 or more drinks per sitting is defined "Binge Drinking", which definitely is an unhealthy behavior. This type of behavior has negative effects on your health on the long and short terms. Physically, alcohol is a source of direct and indirect caloric intake, it can also affect your academic performance and it can potentially have influence on your safety, because of unhealthy consequences, such as drinking and driving or unsafe sex. Remember that not many people is able to totally absorb this amount of alcohol. Also when you feel to be ok, your boy not necessarily has absorbed all the consumed alcohol.

$\mathrm{QM}=Q 34 D+Q 35 B+Q 36 B$

Even if you do not have the perception of having an uncommon drinking habits, drinking 5 or more drinks per sitting is defined "Binge Drinking", which definitely is an unhealthy behavior. In addition to the quantity of alcohol also the frequency of this drinking can be negatively affecting your life. Drinking three or more times in a week is considered "Frequent Drinking". Physically, alcohol is a source of direct and indirect caloric intake, it can also affect your 
academic performance and it can potentially have influence on your safety, because of unhealthy consequences, such as drinking and driving or unsafe sex. Remember that not many people is able to totally absorb this amount of alcohol. Also when you feel to be ok, your boy not necessarily has absorbed all the consumed alcohol.

$\mathrm{RM}=Q 34 D+Q 35 A+Q 36 C \rightarrow I M P O S S I B L E$

$\mathrm{SM}=Q 34 D+Q 35 B+Q 36 C$

Even if you do not have the perception of having an uncommon drinking habits, drinking 5 or more drinks per sitting is defined "Binge Drinking", which definitely is an unhealthy behavior. . When these episodes repeat five or more time in a month, you fit in the category of the "Heavy Drinkers". Physically, alcohol is a source of direct and indirect caloric intake, it can also affect your academic performance and it can potentially have influence on your safety, because of unhealthy consequences, such as drinking and driving or unsafe sex. Remember that not many people is able to totally absorb this amount of alcohol. Also when you feel to be ok, your boy not necessarily has absorbed all the consumed alcohol.

Female

Q34 0=A; 1-2=B; 3-4 = C; 5-10=D

$\mathrm{Q} 35 \leq 2=\mathrm{A} ; \geq 3=\mathrm{B}$

$\mathrm{Q} 360=\mathrm{A} ; 1-3=\mathrm{B} ; \geq 4=\mathrm{C}$

$\mathrm{Q} 34=\mathrm{A}$

Good Job! Sobriety is a good habit! Alcohol consumption can have many negative consequences on your health directly and indirectly.

$\mathrm{AF}=Q 34 B+Q 35 A+Q 36 A$

Very well done! Drinking moderately is a good habit or at least a good way to not over consume it, being exposed to the negative consequences of it. Moderate drinking and drinking in general definitely has a caloric intake effect, and potentially health effects that need to be kept in consideration.

$\mathrm{BF}=Q 34 B+Q 35 B+Q 36 A$

Even if you generally drink moderately. Drinking in general definitely has a caloric intake effect, and potentially health effects that need to be kept in consideration.

Drinking three or more times in a week is considered frequent drinking. This type of behavior has negative effects on your health on the long and short terms. Physically alcohol is a source of direct and indirect caloric intake, it can also affect your academic performance and it can potentially have influence on your safety, because of unhealthy consequences, such as drinking and driving or unsafe sex.

$\mathrm{CF}=Q 34 B+Q 35 A+Q 36 B$

Even if you generally drink moderately. Drinking in general definitely has a caloric intake effect, and potentially health effects that need to be kept in consideration.

Drinking 4 or more drinks per sitting is defined "Binge Drinking", which definitely is an unhealthy behavior. This type of behavior has negative effects on your health on the long and 
short terms. Physically, alcohol is a source of direct and indirect caloric intake, it can also affect your academic performance and it can potentially have influence on your safety, because of unhealthy consequences, such as drinking and driving or unsafe sex. Remember that not many people is able to totally absorb this amount of alcohol. Also when you feel to be ok, your boy not necessarily has absorbed all the consumed alcohol.

$\mathrm{DF}=Q 34 B+Q 35 B+Q 36 B$

Even if you generally drink moderately. Drinking in general definitely has a caloric intake effect, and potentially health effects that need to be kept in consideration.

Drinking three or more times in a week is considered frequent drinking. This type of behavior has negative effects on your health on the long and short terms. Physically alcohol is a source of direct and indirect caloric intake, it can also affect your academic performance and it can potentially have influence on your safety, because of unhealthy consequences, such as drinking and driving or unsafe sex.

Drinking 4 or more drinks per sitting is defined "Binge Drinking", which definitely is an unhealthy behavior. This type of behavior has negative effects on your health on the long and short terms. Physically, alcohol is a source of direct and indirect caloric intake, it can also affect your academic performance and it can potentially have influence on your safety, because of unhealthy consequences, such as drinking and driving or unsafe sex. Remember that not many people are able to totally absorb this amount of alcohol. Also when you feel to be ok, your boy not necessarily has absorbed all the consumed alcohol.

$\mathrm{EF}=Q 34 B+Q 35 A+Q 36 C$

Even if you generally drink moderately. Drinking in general definitely has a caloric intake effect, and potentially health effects that need to be kept in consideration.

Experiencing five or more episodes of Binge Drinking ( 4 or more drinks in a setting) in a month is considered to be "Heavy Drinking". This type of behavior has negative effects on your health on the long and short terms. Physically, alcohol is a source of direct and indirect caloric intake, it can also affect your academic performance and it can potentially have influence on your safety, because of unhealthy consequences, such as drinking and driving or unsafe sex. Remember that not many people are able to totally absorb this amount of alcohol. Also when you feel to be ok, your boy not necessarily has absorbed all the consumed alcohol.

$\mathrm{FF}=Q 34 B+Q 35 B+Q 36 C$

Even if you generally drink moderately. Drinking in general definitely has a caloric intake effect, and potentially health effects that need to be kept in consideration.

Although drinking three or more times in a week is already considered to be "Frequent Drinking", experiencing five or more episodes of Binge Drinking (4 or more drinks in a setting) in a month is considered to be "Heavy Drinking". This type of behavior has negative effects on your health on the long and short terms. Physically, alcohol is a source of direct and indirect caloric intake, it can also affect your academic performance and it can potentially have influence on your safety, because of unhealthy consequences, such as drinking and driving or unsafe sex. Remember that not many people are able to totally absorb this amount of alcohol. Also when you feel to be ok, your boy not necessarily has absorbed all the consumed alcohol. 
$\mathrm{GF}=Q 34 C+Q 35 A+Q 36 A$

Although the general impression can be that drinking three or four drinks per setting can be considered "Moderate Drinking", it is considered "Exaggerated Drinking". This type of behavior has negative effects on your health on the long and short terms. Drinking moreover having effects on the general daily caloric intake, it can also affect your academic performance and it can potentially influence your health.

$\mathrm{HF}=Q 34 C+Q 35 B+Q 36 A$

Although the general impression can be that drinking three or four drinks per setting can be considered "Moderate Drinking", it is considered "Exaggerated Drinking". In addition to the quantity of alcohol also the frequency of this drinking can be negatively affecting your life. Drinking three or more times in a week is considered "Frequent Drinking".

This type of behavior has negative effects on your health on the long and short terms. Drinking moreover having effects on the general daily caloric intake, it can also affect your academic performance and it can potentially influence your health.

$\mathrm{JF}=Q 34 C+Q 35 A+Q 36 B$

Although the general impression can be that drinking three or four drinks per setting can be considered "Moderate", it is better defined as "Exaggerated Drinking", and when characterized by episodes during which you drink 4 or more drinks per sitting is defined "Binge Drinking", which definitely is an unhealthy behavior. This type of behavior has negative effects on your health on the long and short terms. Physically, alcohol is a source of direct and indirect caloric intake, it can also affect your academic performance and it can potentially have influence on your safety, because of unhealthy consequences, such as drinking and driving or unsafe sex. Remember that not many people are able to totally absorb this amount of alcohol. Also when you feel to be ok, your boy not necessarily has absorbed all the consumed alcohol.

$\mathrm{KF}=Q 34 C+Q 35 B+Q 36 B$

Although the general impression can be that drinking three or four drinks per setting can be considered "Moderate", it is better defined as "Exaggerated Drinking", and when characterized by episodes during which you drink 4 or more drinks per sitting is defined "Binge Drinking", which definitely is an unhealthy behavior. In addition to the quantity of alcohol also the frequency of this drinking can be negatively affecting your life. Drinking three or more times in a week is considered "Frequent Drinking".

Physically, alcohol is a source of direct and indirect caloric intake, it can also affect your academic performance and it can potentially have influence on your safety, because of unhealthy consequences, such as drinking and driving or unsafe sex. Remember that not many people are able to totally absorb this amount of alcohol. Also when you feel to be ok, your boy not necessarily has absorbed all the consumed alcohol.

$\mathrm{LF}=Q 34 C+Q 35 A+Q 36 C$

Although the general impression can be that drinking three or four drinks per setting can be considered "Moderate", it is better defined as "Exaggerated Drinking", and when characterized by episodes during which you drink 4 or more drinks per sitting is defined "Binge Drinking", which definitely is an unhealthy behavior. When these episodes repeat five or more time in a month, you fit in the category of the "Heavy Drinkers". 
Physically, alcohol is a source of direct and indirect caloric intake, it can also affect your academic performance and it can potentially have influence on your safety, because of unhealthy consequences, such as drinking and driving or unsafe sex. Remember that not many people are able to totally absorb this amount of alcohol. Also when you feel to be ok, your boy not necessarily has absorbed all the consumed alcohol.

$\mathrm{MF}=Q 34 C+Q 35 B+Q 36 C$

Although the general impression can be that drinking three or four drinks per setting can be considered "Moderate", it is better defined as "Exaggerated Drinking", and when characterized by episodes during which you drink 4 or more drinks per sitting is defined "Binge Drinking", which definitely is an unhealthy behavior. When these episodes repeat five or more time in a month, you fit in the category of the "Heavy Drinkers". In addition to the quantity of alcohol also the frequency of this drinking can be negatively affecting your life. Drinking three or more times in a week is considered "Frequent Drinking".

Physically, alcohol is a source of direct and indirect caloric intake, it can also affect your academic performance and it can potentially have influence on your safety, because of unhealthy consequences, such as drinking and driving or unsafe sex. Remember that not many people are able to totally absorb this amount of alcohol. Also when you feel to be ok, your boy not necessarily has absorbed all the consumed alcohol.

$\mathrm{NF}=Q 34 D+Q 35 A+Q 36 A \rightarrow I M P O S S I B L E$

$\mathrm{OF}=Q 34 D+Q 35 B+Q 36 A \rightarrow I M P O S S I B L E$

$\mathrm{PF}=Q 34 D+Q 35 A+Q 36 B$

Even if you do not have the perception of having an uncommon drinking habits, drinking 4 or more drinks per sitting is defined "Binge Drinking", which definitely is an unhealthy behavior. This type of behavior has negative effects on your health on the long and short terms. Physically, alcohol is a source of direct and indirect caloric intake, it can also affect your academic performance and it can potentially have influence on your safety, because of unhealthy consequences, such as drinking and driving or unsafe sex. Remember that not many people is able to totally absorb this amount of alcohol. Also when you feel to be ok, your boy not necessarily has absorbed all the consumed alcohol.

$\mathrm{QF}=Q 34 D+Q 35 B+Q 36 B$

Even if you do not have the perception of having an uncommon drinking habits, drinking 4 or more drinks per sitting is defined "Binge Drinking", which definitely is an unhealthy behavior. In addition to the quantity of alcohol also the frequency of this drinking can be negatively affecting your life. Drinking three or more times in a week is considered "Frequent Drinking". Physically, alcohol is a source of direct and indirect caloric intake, it can also affect your academic performance and it can potentially have influence on your safety, because of unhealthy consequences, such as drinking and driving or unsafe sex. Remember that not many people is able to totally absorb this amount of alcohol. Also when you feel to be ok, your boy not necessarily has absorbed all the consumed alcohol.

$\mathrm{RF}=Q 34 D+Q 35 A+Q 36 C \rightarrow I M P O S S I B L E$ 
$\mathrm{SF}=Q 34 D+Q 35 B+Q 36 C$

Even if you do not have the perception of having an uncommon drinking habits, drinking 4 or more drinks per sitting is defined "Binge Drinking", which definitely is an unhealthy behavior. . When these episodes repeat five or more time in a month, you fit in the category of the "Heavy Drinkers". Physically, alcohol is a source of direct and indirect caloric intake, it can also affect your academic performance and it can potentially have influence on your safety, because of unhealthy consequences, such as drinking and driving or unsafe sex. Remember that not many people is able to totally absorb this amount of alcohol. Also when you feel to be ok, your boy not necessarily has absorbed all the consumed alcohol.

$\underline{T T M}$

Q37

$1 \rightarrow$ Precontempletion

While sobriety can have many benefits to avoid unnecessarily caloric intake and potential indirect health related negative consequences, also drinking in moderation can have on the longterm positive consequences. Although studies have shown that moderate drinkers tend to live longer than those who either abstain or drink heavily and that moderate alcohol consumption reduces heart attacks, ischemic strokes and circulatory problems through a number of identified ways, these benefits are not found in youth but only with older adults.

Among many of the pros of drinking in moderation or not drinking at parties or club, is the possibility to avoid unhealthy behaviors, to socialize with people and find new friends. No drinking you can be sure to be able to take care of your friends and to avoid to get potentially in trouble.

At WVU you can find numerous alternative to drinking-based activity such as movie theaters, the ice skating ring, and the weekly UpAllNight at the Mountainlair. When you want to go to parties you can alternate alcohol based drinks, with non-alcoholic drinks or limit the number of drinks per night. Another great idea is to organize "non-alcoholic" parties.

\section{$2 \rightarrow$ Contemplation}

While sobriety can have many benefits to avoid unnecessarily caloric intake and potential indirect health related negative consequences, also drinking in moderation can have on the longterm positive consequences. Although studies have shown that moderate drinkers tend to live longer than those who either abstain or drink heavily and that moderate alcohol consumption reduces heart attacks, ischemic strokes and circulatory problems through a number of identified ways, these benefits are not found in youth but only with older adults.

Among many of the pros of drinking in moderation or not drinking at parties or club, is the possibility to avoid unhealthy behaviors, to socialize with people and find new friends. No drinking you can be sure to be able to take care of your friends and to avoid to get potentially in trouble.

Often we associate alcohol with our ability to be "social" and with the only way to be able to fit, but many are the opportunity to avoid this feelings, such alternative to drinking-based activity such as movie theaters, the ice skating ring, and the weekly UpAllNight at the Mountainlair. When you want to go to parties you can alternate alcohol based drinks, with non-alcoholic drinks or limit the number of drinks per night. Another great idea is to organize "non-alcoholic" parties. 
Some feelings of frustration at the beginning due to the peer pressure and the perceived lack of alternative is a common experience. Honestly talking to your friends and social support system about your idea of moderate the drinking habit, can be a great beginning point. They might be able to help you in changing this behavior. Although it seems almost impossible to fight all the perceived barriers you perceive you'll have to face, if you will plan how to overcome them everything will become much easier. Planning is often much more important than being motivated.

\section{$3 \rightarrow$ Preparation}

Often we associate alcohol with our ability to be "social" and with the only way to be able to fit, but many are the opportunity to avoid this feelings, such alternative to drinking-based activity such as movie theaters, the ice skating ring, and the weekly UpAllNight at the Mountainlair. When you want to go to parties you can alternate alcohol based drinks, with non-alcoholic drinks or limit the number of drinks per night. Another great idea is to organize "non-alcoholic" parties. Some feelings of frustration at the beginning due to the peer pressure and the perceived lack of alternative is a common experience. Honestly talking to your friends and social support system about your idea of moderate the drinking habit, can be a great beginning point. They might be able to help you in changing this behavior. Although it seems almost impossible to fight all the perceived barriers you perceive you'll have to face, if you will plan how to overcome them everything will become much easier.

Because planning is often much more important than being motivated, to be willing to change is a very good step, but it needs to be followed by other steps. It is relevant to spend some time in thinking about how to reach this change, which barriers you will eventually meet and how you could overcome them. While thinking about this change, it is important to consider that some difficult moments and small slips will happen.

\section{$4 \rightarrow$ Action}

Some feelings of frustration at the beginning due to the peer pressure and the perceived lack of alternative is a common experience. Honestly talking to your friends and social support system about your idea of moderate the drinking habit, can be a great beginning point. They might be able to help you in changing this behavior. Although it seems almost impossible to fight all the perceived barriers you perceive you'll have to face, if you will plan how to overcome them everything will become much easier.

Because planning is often much more important than being motivated, to be willing to change is a very good step, but it needs to be followed by other steps. It is relevant to spend some time in thinking about how to reach this change, which barriers you will eventually meet and how you could overcome them. While thinking about this change, it is important to consider that some difficult moments and small slips will happen.

Rewarding your effort in changing this behavior can be a very useful strategy to become consistent. An example of this rewarding could be planning to a party once every two weeks, and being careful to not be driving and to be drinking moderately, let yourself drink your favorite drinks.

A great way to be consistent with your health behavior consists in substituting your drinking habits or moments with healthy alternatives. Another idea it could be to limit the drinking nights with for example to go to the movie or to dinner with friends, or some other among the opportunity that Morgantown offers. Another strategy s to avoid to be exposed to alcohol based 
drinks, having them around, make your change much harder. In doing this you can also ask for help to your friends. Sharing with them your desire to maintain a moderate drinking habit can be an important first step, and to build new relationship with other moderate drinkers can be important as well..

\section{$5 \rightarrow \quad$ Maintenance}

Rewarding your effort in changing this behavior can be a very useful strategy to continue being consistent in your behavior. An example of this rewarding could be planning to a party once every two weeks, and being careful to not be driving and to be drinking moderately, let yourself drink your favorite drinks. Or to plan a no drinking dinner in your favorite restaurant, with the money you do not spend in alcohol you can pay a great dinner.

Keep finding healthy alternatives and meeting moderate drinking or no drinking friends are great ways to continue your behavior.

Intention to Behave

$Q 38 \leq 4 \rightarrow Q 39+Q 40+Q 41$

$Q 38 \leq 4$ and/or $Q 42 \leq 4 \rightarrow$

Moderate drinking is an important behavioral habit. Although studies have shown the positive consequences of moderate drinking on health, these are seen only in older adults. Moderate alcohol consumption can help you to not exaggerate the caloric intake due to alcohol or to food to compensate the alcohol consumption (late meals or unhealthy food). Alcohol consumption, if not in moderation, can have long and short term consequences on your health and wellness. These consequences can be direct, such as the alcohol intoxication, or indirect, such as drinking and driving. The consumption of alcohol can have also negative consequences on our social skills (trash talks, unfaithfulness), on our discerning skills (unsafe sex, sex with unknown people, location to go to, etc), and on our immediate choices (overreaction to cops or questionable behaviors)

$Q 42>2$

Well done! It seems that to you to have a healthy drinking habit is important!

$Q 43, Q 44, Q 45, Q 46 \rightarrow$ In any case give WVU Statistics acha-ncha

Q47 if Yes $\rightarrow$ Feedback for each barrier

a. Try to fight peer pressure $\rightarrow$ surround yourself $w /$ moderate drinkers

Sometimes the hardest barrier to overcome is the pressure to drink that our peer execute on us. Surrounding yourself with people that as you made the decision to moderate or quit their drinking habit, can be a first step to resist this pressure. Another way to do so is represented also by your asking your friend for support in your choice, asking them to be your ally in your behavior.

b. example of other ways to manage stress

Sometimes we assume that to manage stress we can drink, but besides being a false myth, there are many more effective alternatives tat can be used to manage stress. The Office of Student Affairs offer workshop to learn how to manage stress or you can find info online. Being physically active is among the most effectives stress relieving activity. In top of these there are 
also other alternatives, such as planning the week in order to be able to manage with all your work or finding hobbies (i.e., play guitar, play videogames, reading, walking the dog, etc) that can provide you with some relaxing time.

c. entertaining and fun alternative activities in town

In Morgantown, many are the possible alternatives to drinking-based events. UpAllNight at the Mountainlair, The movie theaters, the ice-ring, non-alcoholic house parties, bowling structures, many restaurants. In top of these opportunities you can also go to clubs and bars, where, although a little harder to maintain a moderate drinking behavior, if planned, for example drinking only two alcoholic drinks, or less, and drinking non-alcoholic drink, it can be very good and healthy time.

d. alternative place to socialize with people (clubs, and activities)

In Morgantown, many are the possible alternatives to drinking-based events. UpAllNight at the Mountainlair, the movie theaters, the ice-ring, non-alcoholic house parties, bowling structures, many restaurants.

$\underline{\text { e. } a+d}$

Sometimes the need to fit in is going along with the pressure we perceived from our peers. Surrounding your self with people that as you made the decision to moderate or quit their drinking habit, can be a first step to resist this pressure ad to feel belonging. A good way to meet these people (if you do not know them yet) is to go to places where you can spend some quality time, not necessarily drinking such as no drinking parties, UpAllNight at the Mountainlair, the movie theaters, the ice-ring, non-alcoholic house parties, bowling structures, many restaurants. f. Differences b/w perceived social norms and reality (use acha-ncha)

Here in Morgantown we perceived that everybody drinks and that the amount of this alcohol consumption is extremely high. This perception is accompanied by the reality which shows how instead not everybody drinks and overall not everybody drinks as much as we think. The ACHANCHA survey showed that almost $8 \%$ of the WVU students did not drink in the last 30 days and that only $11 \%$ drank between 10 and 30 days within the last month. In contrast of these data the same students perceived that over the $80 \%$ of themselves has drunk 10 to 30 times in the last 30 days. A third of the WVU students stated to drink less than 4 drinks per setting in a party and $38 \%$ of them never binge drinking in the last 15 days.

\section{$Q 43$ to $Q 46$}

The $8 \%$ of WVU students did not drink during the last 30 days, and only the $11 \%$ of it drank more than 10 times during the same period of time. Although these are the numbers, these students think that no one stay sober and that over the $80 \%$ of the WVU students drank more than 10 times. Looking at this it is clear how the perception of the social norms we have of WVU does not represent the real behaviors acted by the students.

Over a third of these students did not experience binge drinking last time they partied and almost the $40 \%$ did not experienced it in the last 15 days. Only the $20 \%$ as binge drunk more than three times in the last fifteen days.

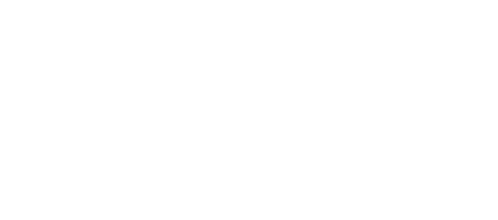

